\title{
The very low-density lipoprotein-triacylglycerol pathway and metabolic heterogeneity in familial combined hyperlipidemia
}

Citation for published version (APA):

Georgieva, A. M. (2005). The very low-density lipoprotein-triacylglycerol pathway and metabolic heterogeneity in familial combined hyperlipidemia. [Doctoral Thesis, Maastricht University]. Universiteit Maastricht. https://doi.org/10.26481/dis.20051103ag

Document status and date:

Published: 01/01/2005

DOI:

10.26481/dis.20051103ag

Document Version:

Publisher's PDF, also known as Version of record

Please check the document version of this publication:

- A submitted manuscript is the version of the article upon submission and before peer-review. There can be important differences between the submitted version and the official published version of record.

People interested in the research are advised to contact the author for the final version of the publication, or visit the DOI to the publisher's website.

- The final author version and the galley proof are versions of the publication after peer review.

- The final published version features the final layout of the paper including the volume, issue and page numbers.

Link to publication

\footnotetext{
General rights rights.

- You may freely distribute the URL identifying the publication in the public portal. please follow below link for the End User Agreement:

www.umlib.nl/taverne-license

Take down policy

If you believe that this document breaches copyright please contact us at:

repository@maastrichtuniversity.nl

providing details and we will investigate your claim.
}

Copyright and moral rights for the publications made accessible in the public portal are retained by the authors and/or other copyright owners and it is a condition of accessing publications that users recognise and abide by the legal requirements associated with these

- Users may download and print one copy of any publication from the public portal for the purpose of private study or research.

- You may not further distribute the material or use it for any profit-making activity or commercial gain

If the publication is distributed under the terms of Article $25 \mathrm{fa}$ of the Dutch Copyright Act, indicated by the "Taverne" license above, 


\title{
The Very Low-Density Lipoprotein- Triacylglycerol Pathway and Metabolic Heterogeneity in Familial Combined Hyperlipidemia
}

\author{
PROEFSCHRIFT \\ ter verkrijging van de graad van doctor \\ aan de Universiteit Maastricht, \\ op gezag van de Rector Magnificus, \\ Prof. mr. G.P.M.F. Mols \\ volgens het besluit van het College van Decanen, \\ in het openbaar te verdedigen \\ op donderdag 3 november 2005 om 16.00 uur
}

door

Anna Marinova Georgieva 
Promotores:

Copromotores:

Dr. M.M.J. van Greevenbroek

Dr. C.J.H. van der Kallen

Beoordelingscommissie: Prof. dr. C.D.A. Stehouwer (woorzitter)

Prof. dr. M.J.A.P. Daemen

Prof. dr. J.F.C. Glatz

Prof. dr. M.J. Hofker

This work was financially supported by a Marie Curie Fellowship of the European

Community program "Quality of Life and Management of Living Resources" (contract

number QLK5-CT-2000-60007), Maastricht University, Cardiovascular Research Institute Makstricht (CARIM), Job Cohen Fund, and Pfizer BV.

Financial support by the Netherlands Heart Foundation and J.E. Jurriaanse Stichting for the publication of this thesis is gratefully acknowledged.

Printing of this thesis was financially supported by Pfizer B.V., Merck Sharp \& Dohme B.V., and Astra Zeneca.

(c) Anna M. Georgieva, 2005

ISBN-10: 90-9019-777-X

ISBN-13:97-8909-019-7777

Design Dessislava Staneva

Printed by Ponsen \& Looijen B.V., Wageningen 
На моето семейство, с много обич.

To my family with love. 



\section{Contents}

\section{List of abbreviations}

Chapter 1

General introduction: Metabolic dissection of familial combined hyperlipidemia. Focus of this thesis.

Chapter 2

Subclasses of low-density lipoprotein and very low-density lipoprotein in famillal combined hyperlipidemia: relation to multiple lipoprotein phenotype phenomenon.

Chapter 3

Radiological evidence of nonalcoholic fatty liver disease in familial combined hyperlipidemia.

Chapter 4

Prothrombotic markers in familial combined hyperlipidemia. Evidence of endothelial cell activation and relation to metabolic syndrome.

Chapter 5

Very low-density lipoprotein subclass distribution in familial combined hyperlipidemia; relationship to hypertriglyceridemia and metabolic syndrome

Chapter 6

Lipitor, Inflammation and Gene Expression in Adipose Tissue (LIGEAT) in familial combined hyperlipidemia. I. Effect on plasma lipid and lipoprotein profile, and insulin resinstance.

Chapter 7

General discussion

Appendix

Summary and Samenvatting

Acknowledgements

List of publications 


\section{List of abbreviations:}

Apo apolipoprotein

ARF $-1 \quad$ ADP ribosylation factor 1

CAD coronary artery disease

CE cholesteryl ester

CETP cholesteryl ester transfer protein

CM chylomicrons

DAG diacylglycerol

FCHL familial combined hyperlipidemia

FFA free fatty acids

HDL high-density lipoprotein

HL hepatic lipase

HSL hormone sensitive lipase

IDL intermediate-density lipoprotein

IMT intima media thickness

LDL low-density lipoprotein

LPL $\quad$ lipoprotein lipase

LRP LDL receptor related protein

LXR liver $X$ receptor

PAI-1 plasminogen activator inhibitor 1 .

PLD phospholipase D

Sd LDL $\quad$ small dense LDL

SREBP sterol response element binding protein

sTF soluble tissue factor

sTM soluble Thrombonodulin

TAT thrombin-antithrombin complex 


$\begin{array}{ll}\text { TG } & \text { triglycerides, triacylglycerols } \\ \text { TC } & \text { total cholesterol } \\ \text { t-PA } & \text { tissue-derived plasminogen activator } \\ \text { TRL } & \begin{array}{l}\text { triglyceride-rich lipoproteins } \\ \text { thiazolidinediones }\end{array} \\ \text { TZD } & \\ \text { VLDL } & \text { very low-density lipoprotein } \\ \text { WWF } & \text { von Willebrand factor } \\ \text { WHR } & \text { waist-to-hip ratio } \\ \text { USFI } & \text { upstream stimulating factor } 1 \\ \text { F XII } & \text { factor XII activated }\end{array}$



Chapter 1

\section{General Introduction}

Metabolic Dissection of Familial Combined Hyperlipidemia 


\section{Contents:}

\section{Introduction}

1.1 Metabolic profile of FCHL

1.2 Metabolic background

Box l: Physiology of fatty acid metabolism in adipose tissue.

\section{Organ insulin resistance and lipid metabolism in FCHL}

2.1 Disturbed adipose tissue metabolism contributes to elevated plasma levels of FFA in FCHL

2.2 Consequences of increased FFA flux

2.2.1 Increased FFA flux and insulin resistance in skeletal muscle.

2.2.2 Metabolically challenged liver in FCHL

2.3 Hepatic lipoprotein production

Box II: Plasma lipoproteins

2.3.1 Molecular mechanisms of hepatic lipoprotein production

3. Hyperlipidemia in $\mathrm{FCHL}$

4. Scope of this thesis 


\section{Introduction}

\subsection{Metabolic Profile of FCHL}

Familial Combined Hyperlipidemia (FCHL) was delineated three decades ago as a genetic disorder of lipid metabolism occurring in survivors of a myocardial infarction and their relatives ${ }^{t}$. The initial study showed that approximately $10 \%$ of the myocardial infarction survivors exhibited familial hyperlipidemia, characterized by wariable expression within kindred: i.e. isolated hypercholesterolemia (Fredrickson IIa), combined hyperlipidemia (IIb), or isolated hypertriglyceridemia (IV), whereas other relatives were normolipidemic. A proportion of affected individuals (up to $25 \%$ ) changes phenotype over time ${ }^{1.2}$. Subsequent studies have shown that affected FCHL relatives can present with most of the features of the metabolic syndrome: elevated plasma insulin concentrations ${ }^{3,4}$ in the presence of insulin resistance ${ }^{5-8}$, visceral obesity ${ }^{9,10}$, hypertension ${ }^{3,10}$, as well as netabolic dyslipidemia, including small dense low-density lipoproteins (sd LDL), decreased plasma high-density lipoprotein (HDL) cholesterol concentrations, and increased plasma Apo B levels ${ }^{3.11}$. Elevated Apo B levels, which reflect an increased number of lipoprotein particles in plasma, are considered inherent to FCHL ${ }^{12,13}$.

\subsection{Metabolic Background}

Despite numerous metabolic and genetic studies, the pathophysiology and the genetic background of FCHL remain a matter of debate. Hyperlipidemia, an overt manifestation of FCHL, results from accumulation of Apo B-48 and Apo B-100 containing lipoprotein particles in plasma, especially in the postprandial state ${ }^{14}$. Hence, a significant part of scientific research has focused on the plasma compartment in FCHL. Genetic analysis showed evidence that factors that control plasma lipoprotein metabolism, such as Apo $\mathrm{Al}^{15}$, Apo All ${ }^{16}$, Apo $\mathrm{CIII}^{15}$, Apo B ${ }^{17}$, Apo $\mathrm{AV}^{15}$, lipoprotein lipase $(\mathrm{LPL})^{18}$, hepatic lipase $(\mathrm{HL})^{18}$, and cholesterol ester transfer protein (CETP) ${ }^{19}$ are implicated in the etiology of FCHL as susceptibility genes. These genes modify the expression of the FCHL phenotype. 
Plasma lipid levels represent the result of acute adaptations (for instance in response to meals), and long-term adaptations to changes in energy homeostasis. Several organs that are implicated in lipid metabolism play an important role in this adaptation, i.e. adipose tissue, liver and skeletal muscle (Figure 1; Box I). These organs direct the flux of energy substrates, glucose and free fatty acid, FFA, in the body in response to insulin. In FCHL, adipose tissue and muscle exhibit an impaired endocrine response to insulin, meaning that these tissues are insulin resistant ${ }^{5,6,8}$. There is no evidence in the literature yet to suggest that insulin resistance in liwer contributes to FCHL, although this is quite likely. For example, the liver has been implicated in other disorders with insulin resistance, such as type $2 \mathrm{DM}^{20}$. In the following sections, we will review lipid metabolism in FCHL in relation to organ function. We will focus on the role of FFA fluxes and insulin; and cliscuss hyperlipidemia in FCHL.

Box I Physiology of Fatty Acid Metabolism in Adipose Tissue. Fatty acids constitute an important fuel for many organs in the body. However, at increased concentrations fatty acids can have diabetogenic, or even toxic effects ${ }^{21}$. Intracellular accumulation of fatty acids as triglycerides can impede insulin action (as shown in muscle and liver) ${ }^{22.24}$, or in some cases, it can induce apoptosis. This latter mechanism has been proposed as an explanation of apoptosis of the beta-cells of the pancreas $^{21}$. Homeostatic mechanisms prevent increased systemic FFA concentrations. For instance, at times of energy surplus, FFA along with glucose, which is converted to glycerol in the adipocyte, can be stored as triacylglycerols in adipose tissue. The storage potential of adipose tissue can serve as a buffer that protects other organs against the deleterious effect of thigh plasma FFA ${ }^{25}$.

The buffer capacity of subcutaneous adipose tissue is determined by the rate of lipolysis and lipogenesis (esterification) of triacylglycerols (Figure 1) $)^{26}$. These metabolic parhways are modified by genetic variation and are fine-tuned by a variety of homonal and nutritional factors. Insulin promotes lipogenesis in adipose tissue and acutely suppresses lipolysis via inhibition of the enzyme hormone sensitive lipase (HSL). The postprandial increase in insulin levells potentiates storage of faty acids in adipose tissue, and thus prevents extreme plasma concentrations. Several other hormones, e.g. catecholamines, glucagon and growth hormone antagonize insulin's actions, and are known as lipolytic hormones. The resultant of lipolysis and esterification, or in other words the net result of these regulatory processes, determines the nonesterified fatty acid pool in adipose tissue, and contributes to plasma FFA levels ${ }^{25.27}$. Of note, disturbed FFA metabolism in adipose tissue plays a role in various metabolic diseases. Excessive lipolysis or reduced esterification contribute to elevated plasma FFA levels in type 2 DM and obesity ${ }^{27}$. 


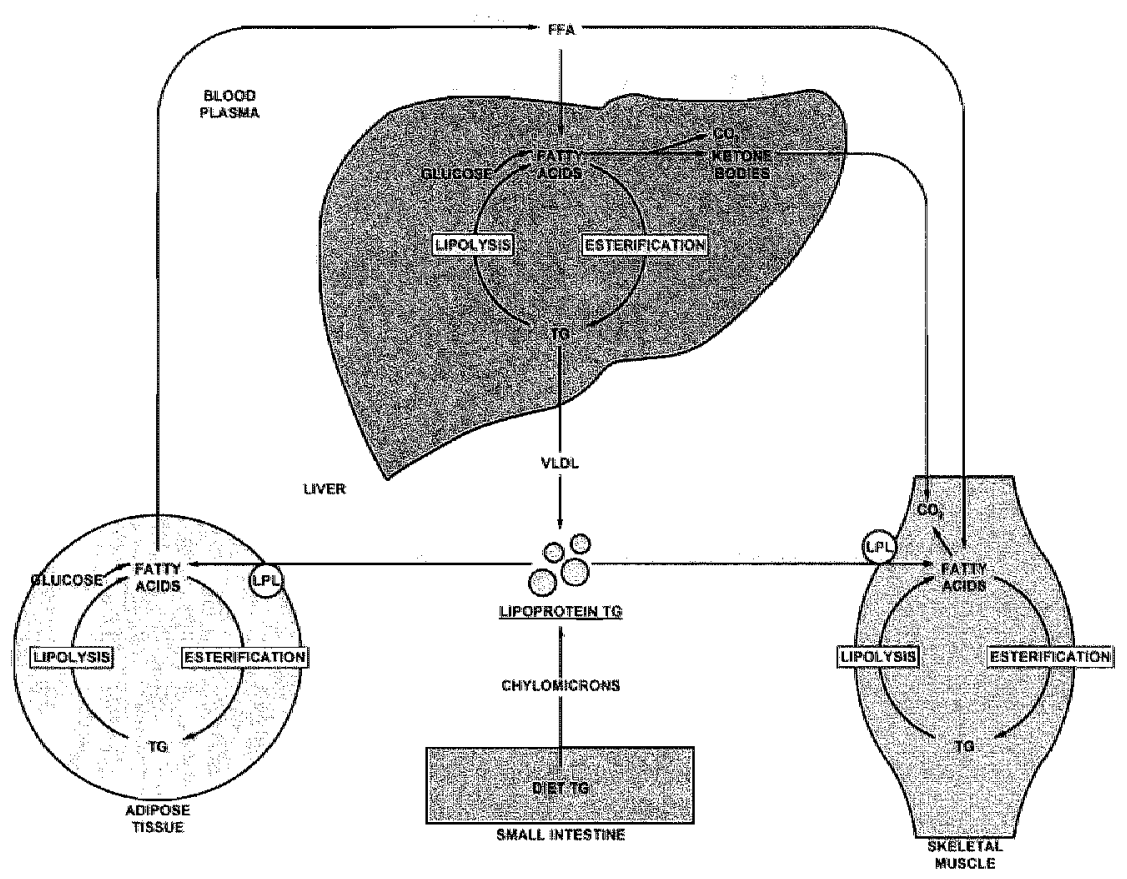

Figure 1. Schematic overview of lipid metabolism, with focus on FFA fluxes.

In the postprandial state, dietary fatty acids are transported as Chylomicrons-TG in the blood for storage (adipose tissue), or as fuel supply (muscle). The excess of FFA is accommodated by the liver and oxidized, or stored as TG. In the fasting state, liver maintains adequate fuel supply to other organs by secretion of FFA as very low-density lipoproteintriacylglycerol (VLDL-TG).

Lipid metabolism in FCHL: 1) delayed clearance of TG-rich lipoproteins in the postprandial state because of relative LPL deficiency; 2) impaired FFA trapping for TG synthesis in adipose tissue; 3) increased FFA flux to liver and muscle; 4) excess hepatic production of fatty acids as VLDL-TG; 5) increased fatty acid oxidation in muscle. 


\section{Organ Insulin Resistance and Lipid Metabolism in FCHL}

\subsection{Disturbed Adipose Tissue Metabolism Contributes to Elevated Plasma Levels of FFA in FCHI}

A number of studies provide evidence that adipose tissue in FCHL shows an impaired response to insulin. FCHL subjects have an extended postprandial increase of FFA, that gradually drops to normal in the postabsorptive state ${ }^{6,7,14}$. Insulin infusion during an euglycemic hyperinsulinemic clamp, lowers plasma FFA and glycerol in controls, but in FCHL subjects they remain elevated ${ }^{6-8,28}$ "Elevated plasma FFA levels can result from insulin resistant (excessive) lipolysis in adipose tissue, associated with increased activity of $\mathrm{HSL}^{27}$. Such a mechanism is operational in type 2 DM, but could not be proven in FCHL ${ }^{8,18.29,30}$. On the contrary, several studies on HSL activity in FCHL demonstrated reduced activity of this enzyme, at least in in vitro studies ${ }^{8,} 39,30$. The latter seems to be an adaptiwe mechanism rather than at metabolic defect, because genetic studies have shown no linkage of the HSL gene locus $(19 \mathrm{q} 13.1 \mathrm{qq} 13.2)$ to the FCHL phenotype 8.18 .31 .

Elevation of fasting FFA, however, can be particularly characteristic for FCHL subjects in the highest triglyceride tertile (TG>2.5 mmol/L) ${ }^{4 / 14}$. These subjects are characterized by the highest BMI as well ${ }^{1 / 4}$. Increased adipose tissue mass, as assessed by BMI, contributes to dyslipidemia, probably through chronically elevated FFA fluxes ${ }^{32}$. In addition, hormones and cytokines, such as leptin, adiponectine, IL- 6 and TNF- $\alpha$, produced by adipose tisste in proportion to adipose tissue mass, can influence whole body insulin resistance and the insulin resistance of adipose tissue in particular. Noteworthy, we have observed a number of differences in gene expression of inflammatory and insulin resistant genes between mature adipocytes of FCHL subjects and controls, including TNF-a (13-fold elevated) and $1 \mathrm{~L}-6(2-\text { fold })^{33}$.

Genetic and metabolic heterogeneity with regard to clearance of triglyceride-rich lipoproteins and FFA metabolism in adipose tissue have been observed withim FCHL ${ }^{34}$. These differences can additionally modify FFA levels. For example, delayed clearance of very low density lipoproteins (VLDL), VLDL remmants and chylomicrons, as observed in hypertriglyceridemic FCHL subjects, can contribute to the increase of FFA, both fasting and 
postprandial, probably through increased intravascular lipolysis ${ }^{14,35}$. In addition, genetic variations in PPAR- $\gamma$ can significantly affect plasma concentrations of fatty acid and glycerol in $\mathrm{FCHL}^{34}$.

A primary genetic defect, associated with impaired fatty acid metabolism in adipose tissue is plausible in FCHL $\mathrm{L}^{6-8}$. Studies of our group and others suggest that such a defect is mostly likely associated with impaired fatty acid trapping for triacylglyceroll synthesis in $\mathrm{FCHL}^{8}$. Others have proposed reduced postprandial fatty acid transport into the adipocyte via acylation-stimulating protein, $\operatorname{ASP}^{36,37}$. However, studies in Finnish families provide evidence against a possible contribution of ASP to the lipid abnormalities in $\mathrm{FCHL}^{3.8}$. Alternatively, we found that the expression of $\mathbb{F A B P 4}$, a fatty acid translocase necessary for the transport of long-chain fatty acids is upregulated in FCHL adipocytes. In addition, CD36, another fatty acid transporter, was upregulated in FCHL adipocytes as well as in preadipocytes $^{39}$. These latter findings probably represent an adaptive response to defective triacylglycerol storage in FCHL adipocytes, and studies to the underlying gene defects are ongoing.

\subsection{Consequences of Increased FFA Fhux}

\subsubsection{Increased FFA Flux and Insulin Resistance in Skeletal Muscle}

Skeletal muscle is responsible for $85 \%$ of the whole body's gllucose uptake. Insullin mediates this process by promoting the availability of glucose transporter GLUT -4 , as well aS the rate of glucose storage in muscle, i.e. glycogen synthesis. Plenty of clinical and biochemical evidence has been gathered that glucose uptake in response to insulin is impaired in FCHL ${ }^{6-8}, 40$. Skeletal muscle metabolism in FCHL appears to differ from controls: it shows a higher rate of lipid oxidation, and lower rate of glucose oxidation than healthy controls, as measured by indirect calorimetry ${ }^{16}$. This can result from the increased FFA supply to muscle in FCHL, and subsequently greater relative use of FFA, instead of glucose, for oxidation, vid the Rancle's cycle ${ }^{4 !}$ (Figure 1). Alternatively, increased FFA uptake in skeletal muscles can ensue from upregulation of fatty acid transporting proteins, as observed in FCHL adipose 
tissue (CD36 and FABP4, as discussed above), and therefore be specific for FCHL. Increased fatty acid uptake can lead to accumulation of fatty acid metabolites in muscle, which can interfere with the intracellular irisulin-signaling cascade ${ }^{22,42-44}$. The extent of muscle insulin resistance in FCHL might be dependent on the rate of FFA supply, or altematively have a genetic basis. Literature suggests that insulin resistant glucose uptake in muscle is observed. predominantly in hypertriglyceridemic FCHL subjects, and is less common in FCHL subjects with hypercholesterolemia only ${ }^{26}$. Failure of insulin's action to stimulate glucose uptake in FCHL muscle can lead to increased plasma glucose concentrations, for which then compensatory hyperinsulinemia is required to maintain euglycemia. Moreover, reduced utilization of glucose by muscle can result in an increased glucose flux to liver, where it can be subsequently stored as glycogen, or converted to triglycerides.

\subsubsection{Metabolically Challenged Liver in FCHL}

Clinical and biochemical data on liver function in FCHL is scarce. However, literature data indicate that liver can have an important contribution to the expression of the disorder.

An important function of liver is to maintain the homeostasis of body fuels: glucose and FFA. At times of energy surplus, liver temporary accommodates the excess of plasma glucose or FFA, and thus can prevent other tissues from fat (energy) overload ${ }^{26}$ (Figure 1). In liver, FFA cain be oxidized oir, under hyperinsulinemic conditions, stored as fat (lipogenesis) 45. This buffer function of liver can be particularly important in FCHL, where adipose tissue fatty acid storage may be compromised ${ }^{7,8}$. Additionally, increased flux of glucose to liver is expected due to diminished glucose utillization in muscle, as described above.

Hepatic lipid metabolism has not been addressed in FCHL. Of interest, it was recently shown that FCHL is associated with the gene encoding upstream regulating factor 1 , USF-1 $1^{46}$. This transcription factor can upregulate the transcription of several genes involved in lipogenesis de nowo in response to high concentrations of intracellular glucose (glucoseresponsive genes $)^{45,474 \%}$. USF-1 may represent a switch between glucose and fat metabolism. Lipogenesis can be potentiated by hyperinsulinemia, because insulin upregulates several insulin-responsive genes with a central role in hepatic lipid metabolism, such as Liver $X$ 
receptor $(\mathrm{LXR})^{50}$, and Sterol Response Element Binding Proteins (SREBPs) ${ }^{51}$. Thus, substrate excess, un combination with hyperinsulinemia can stimulate lipogenesis de novo (i.e. fatty acid synthesis for incorporation in TG) in the liver in FCHL. Enhanced lipogenesis is proposed as one of the mechanisms that laads to fat accumulation in liver ${ }^{32,53}$, and therefore FCHL subjects can be susceptible to develop non-alcoholic fatty liver disease (NAFLD). We report on the high prevalence of fatty liver in ambulatory FCHL patients of the Lipid clinic of the Maastricht University Hospital ${ }^{54}$.

\subsection{Hepatic Lipoprotein-Apo B 100 Production}

Insulin promotes lipid storage and suppresses hepatic glucose and very low density lipoprotein (VLDL) secretion after a meal. Alternatively, at times of energy need, liver can mobilize glucose, or FFA that can be secreted in the form of VLDL-TG. Thus, liver maintains adequate fuel supply to different organs during fasting when plasma insulin levels are low. One important manifestation of hepatic insulin resistance is glucose overproduction, as a result of unsuppressed gluconeogenesis. Another consequence of hepatic insulin resistance can be VLDL overproduction (for plasma lipoproteins see Box II).

Kinetic studies have demonstrated that hepatic overproduction of VLDL particles can explain, at least in part, the frequently observed elevated plasma triglyceride levels in FCHL ${ }^{13}$. The driving mechanism of this overproduction is not known, but literature suggests that hepatic VLDL-Apo B secretion can be a function of hepatic insulin sensitivity ${ }^{55-57}$. The importance of hepatic insulin resistance to triglyceride-rich VLDL production is illustrated by the observation that impaired insulin suppression of VLDL production contributes to increased lipid levels in type $2 \mathrm{DM}^{20}$. Another factor that can mediate VLDL overproduction is accumulation of fat in the liver (fatty liver) ${ }^{56}$. Additional mechanisms can involve enhanced lipogenesis de novo or intrahepatocellular TG breakdown ${ }^{27,45,58,59}$. 
Dox II. Plasma lipoproteins. Plasma lipoprotens and their metabolism can be sorted into 3 major pathways, which interact with each other:

-VLL pathway ${ }^{72}$ VLDL are triglyceride-rich lipoproteins that are secreted by the liver. In plasma, VLDL are depleted from their triglyceride content via the activity of lipases, the main ones being lipoprotein lipase (LPL, localixed on extrahepatic capillary endothelium), and hepatic lipase (HL, space of Disse in liver). During a half-life of minutes to a few hours, VLDL is successively converted to smaller particles: VLDL remnants, intermediate-density lipoproteins (IDL) and subsequently to LDL. Approximately, half of VLDL is rapidly cleared at its stage as a VLDL remnant, ar IDL via Apo E mediated hepatic uptake. The remainder is progressively delipidated to a $L D$ particle. The clearance of LDL is mediated by $\mathrm{Apo} B 100$, and is comparatively slow (half -1 ife of LDL is 48-72hours $)^{72}$. Noteworthy, VLDL and LDL consist of distinct, physicochemically heterogeneous subclasses, which differ in respect to production, regulation and catabolism ${ }^{73}$. By convention, VLDL comprises VLDLLI and VLDL2; LDL includes at least four subclasses: buoyant LDL (LDLI and LDLII), and small dense LDL (LDLIII and LDLIV). It appears that there is a metabolic channeling between VLDL and LDL subclasses ${ }^{73}$. VLDL1 can be the precursor of small dense LDL (sd LDL), whereas VLDL2 is rapidly catabolized to LDL of nomal density and size (buoyant LDL). Sd LDL is considered to be particularly atherogenic ${ }^{74,75}$. This is attributed to the sbility of sd LDL particles to penetrate relatively easy vascular walls, and their susceptibility to oxidation ${ }^{25}$.

Appendix (Table 1) presents enzymes and apolipoproteins implicated in the VLDL-TG pathway.

$-H D L$ pathway. HDL transports cholesterol from the periphery to liver for excretion via the bile, a process known as the reverse cholesterol transport. A part of HDL cholesterol can be delivered to the liver wia the VL.DL-LDL pathway. This process involves transfer of neutral lipid berween HOL and LDL, or VLDL, and is mediated via CETP. Increased plasma VLDL concentrations can result in an entanced transfer of triglycerides (in exchange of cholesterol esters) to $\mathrm{LDL}$ and HDL, and a deteriorated lipoprotein profile ${ }^{77}$. This involves formation of atherogenic sd LDL and decrease in HDL cholesterol. In addition, HDL serves as a reservoir of apolipoproteins (Apo E and Apo C"s) that are transferred to VLDLs and chylomicrons in plasma and can influence their catabolism.

-Chylomicron pathway. Chylomicrons characterize postprandial lipemia. These particles are lipolyzed by LPL to chylomicron remnants, and therefore compete with triglyceride-rich VLDL for a common lipolytic pathway ${ }^{28}$. 


\subsubsection{Molecular Mechanisms of Hepatic Lipoprotein Production}

Hepatic production of Apo-B 100 containing lipoproteins inwolves two main steps, which take place in the secretory apparatus of the hepatocyte (Figure 2) ${ }^{60,61}$. The first step represents transfer of small amount of lipid (triglycerides and phospholipids) by microsomal triglyceride transfer protein (MTP) to newly synthesized Apo B 100, resulting in the formation of a dense pre-VLDL particle ${ }^{62,63}$. If proper lipidation does not take place, Apo B 100 is sequestrated for degradation by chaperons, such as heat shock protein $70(\mathrm{Hsp} 70)^{63.64}$. These processes take place in the rough endoplasmatic reticulum (RER), but some insulin mediated degradation may also occur post-RER ${ }^{64.65}$. The formation of mature VLDL (second step) comprises acquisition of additional lipid in the terminal junctions between the rough and the smooth endoplasmatic reticulum ${ }^{61,62,65}$. MTP is implicated in the transfer of neutral lipid to the lumen of the smooth endoplasmatic reticulum, although the exact mechanism is not yet known ${ }^{63}$. The process of fusion between the pre-VLDL particle and Apo B-free lipid droplet involves, at least in part, adenosine diphosphate ribosylation factor-1 (ARF-1) -mediated activation of phospholipase D (PLD) ${ }^{62,66}$. Apo-B 100 containing lipoproteins produced by the liver are predominantly VLDL, but the density can range from LDL to large VLDL particles, depending on the amount of lipid available for the maturation step. Overproduction of Apo B 100 as VLDL as well as LDL has been reported in FCHL ${ }^{13,67}$.

Insulin regulates lipoprotein synthesis at several steps; it downregulates the MTP gene expression $^{68}$, it suppresses ARF-11 activity ${ }^{62}$, and it mediates some Apo B degradation ${ }^{6 / 4}$ among others. Insullin resistance of one or more of these pathways may result in enhanced production of VLDL-A po $B{ }^{56,57,62,68-70}$. Accumulation of lipid in the secretory apparatus can be another important factor that drives Apo B overproduction ${ }^{23.27 .69}$.

Most of the present knowledge on the molecular mechanism of VLDL production is derived from in vitro and animal studies, and little is known about these processes in humans. Human hepatic tissue is difficult to obtain for studies, which hinders our knowledge on hepatic lipid metabolism in general, and in FCHL in particular. However, genetic studies or markers of hepatic lipid metabolism can advance our understanding. For example, MTP seems less likely as a genetic cause of VLDL-Apo B overproduction, because no evidence of linkage of MTP locus (4q22-q24) to FCHL has been observed so far " (see Appendix). 


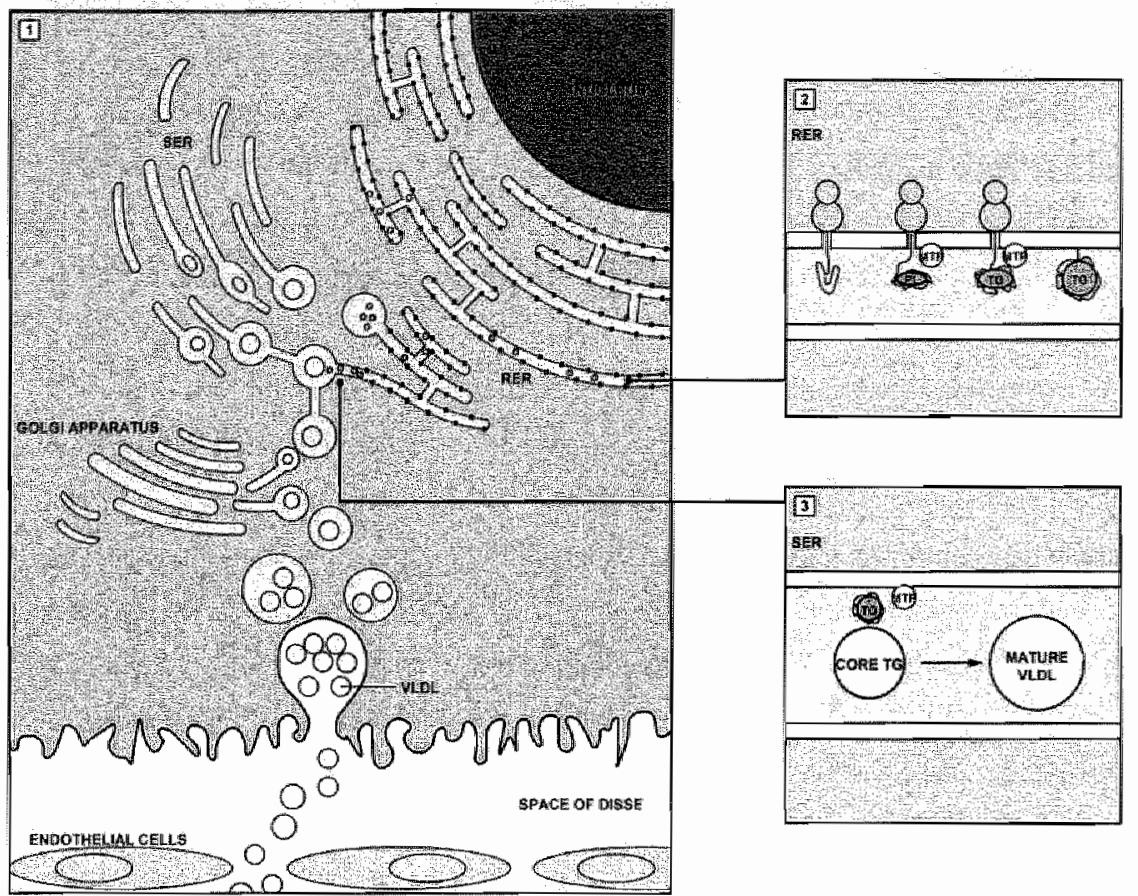

\section{Figure 2. Mechanism of hepatic VLDL-Apo B production}

1) Secretory apparatus of the hepatocyte, $\mathrm{RER}=$ rough endoplasmatic reticulum; SER = smooth endoplasmatic reticulum; 2) The first step of VLDL assembly: Apo B is translated, and simulaneously lipidated by MTP to a pre-VLDL particle in RER, MTP = microsomal triglyceride transfer protein: $\mathrm{PL}=$ phospholipids; $\mathrm{TG}=$ triglycerides; 3 ) The second step of VLDL assembly; the pre-VLDL particle fuses with an Apo-B free lipid droplet to form a mature VLDL particle. 


\section{Hyperlipidemia in FCHL}

The classical phenomenology of hyperlipidemia describes the lipoprotein abnormalities in FCHL as elevation of plasma VLDL (Fredrickson type IV), or LDL (type IIa), or a combination of both (type IIb). Manifestation of different lipoprotein phenotypes among family members is a hallmark of a FCHL family. "This phenomenon has not been fully understood, but genetic and metabolic differences among individuals can be involved.

A more contemporary approach has identified a clustered entity of elewated plasma triglycerides, low HDL cholesterol and predominance of small dense LDL as a phenotype frequently observed in FCHL subjects. In the population, this profile has been named the atherogenic lipoprotein phenotype, because of its association with increased risk for cardiovascular disease ${ }^{79}$. We have studied this adverse phenotype and its metabolic determinants in more detail (chapter 2$)^{80}$.

The following factors are known to contribute to the lipoprotein profile in FCHL:

A. Overproduction: discussed above.

B. Disturbed catabolism of lipoprotein particles in FCHL.

- Impaired clearance by LPL. Postprandial hyperlipidemia is particularly atherogenic $^{81}$. It involves impaired clearance of VLDL, VLDL remnants and chylomicrons, partly because of impaired conversion of chylomicron and large VLDL particles to remnants ${ }^{14,35}$. One explanation is relative insufficiency of LPL, because of comperition for the common lipolytic pathway ${ }^{7 \%}$. Reduced LPL activity, which may have genetic basis ${ }^{82}$, has been reported in a subset of FCHL patients ${ }^{83}$. Although mutations in the LPL do not represent a major gene defect ${ }^{18}$, LPL activity may be an important factor in FCHL, taken into consideration its common polymorphisms and impaired function under conditions of insulin resistance $^{84}$. Furthermore, $L P L$ actiwity reflects PPAR-a activity, because it increases during therapy with fibrates ${ }^{85}$, which are PPAR- $\alpha$ activators. The role of PPAR- $\alpha$ as modifier gene in FCHL has been recently described ${ }^{86}$.

- Other enzymes involved in lipoprore in catabolism. HL and CETP are genetic factors, which contribute to the expression of atherogenic lipoprotein phenotype in FCHL ${ }^{87.88}$.

- Apolipoprotein composition. Surface apolipoproteins determine catabolism and distribution of excess VLDL and VLDL remnants to organs in the body including liver, 
adipose tissue and vascular wall. For example. Apo CII is a cofactor for $L P L$, whereas Apo CIII inhibits LPL activity, at least in vitro. Apo $\mathrm{E}$ mediates hepatic clearance, as well as peripheral uptake and lipid delivery. Association of the Apo AI-CIII-AIV-AV gene cluster with plasma triglyceride levels has been shown in FCHL ${ }^{15}$. However, Apo CIII content of VLDL particles appears normal in $\mathrm{FCHL}^{89}$.

- Heparic remuant clearance can be another factor that contributes to hyperlipidemia in FCHL. FCHL carriers of the Apo E2 allele, tend to have higher triglyceride levels than subjects with the Apo E3 or E4 genotype ${ }^{90}$. Heparic clearance of LDL seems to be relatiwely insufficient as well, at least in some subjects ${ }^{91}$, because treatment with statins, which are known to upregulate hepatic LDL receptors, leads to normalization of plasma $L$ DLcholesterol levels ${ }^{92}$.

All combined, the data clearly indicate that alterations in VLDL pathway can contribute to the expression of FCHL. This pathway carries most of plasma triglycerides in the fasting state, which is the main focus of this thesis. We subsequently refer to it as very low-density lipoprotein-triacylglycerol pathway, VLDL-TG pathway.

\section{Scope of This Thesis}

This thesis focused on the VLDL pathway and hypertriglyceridemia in FCHL. We aimed to identify metabolic factors that can explain the lipoprotein heterogeneity in FCHL. The classical approach to divide FCHL in three major types: Fredrickson Type IIa, IIb and IV on the basis of lipoprotein abnormalities was substituted by simpler one: presence or absence of elevated plasma triglycerides. 


\section{References}

1. Goldstein JL, Schrott HG, Hazzard WR, et al. Hyperlipidentia in coronary heart disease. 1I. Genetic analysis of lipid levels in 176 families and delineation of a new inherited disorder, combined hyperlipidemia. J Clin Invest. Jul 1973;52(7):154441568.

2. Veerkamp MJ, de Graaf $\mathrm{J}$, Bredie SJ, et al. Diagnosis off familial combined hyperlipidemia based on lipid phenotype expression in 32 fanilies: results of a 5 -year follow-up study. Arteriosder Thromb Vasc Biol. Feb 1 2002;22(2):274-282.

3. Hunt SC, Wu LL, Hopkins PN, et al. Apolipoprotein, low density lipoprotein subfraction, and insulin associations with familial combined hyperlipidemia. Study of Utah patients with fanilial dyslipidemic hypertension. Arteriosclerosis. May-Jun 1989;9(3):335-344.

4. Ascaso JF, Sales $\mathrm{J}$, Merchante A, et al. Influence of obesity on plasma lipoproteins, glycaemia and insulinaemia in patients with familial combined hyperlipidaemia. In I Obes Relar Merab Disord. May 1997;21(5):360-366.

5. Ascaso JF, Lorente $\mathrm{R}$, Merchante $\mathrm{A}$, et al. Insulin resistance in patients with familial combined hyperlipidemia and coronary artery disease. Am J Cardiol. Dec 1 1997;80(11):1484-1487.

6. Karjalainen L, Pihlajamaki J, Karhapaa P, et al. Impaired insulin-stimulated glucose oxidation and free fatty acid suppression in patients with familial combined hyperlipidemia: a precursor defect for dyslipidernia? Arterioscler Thromb Vasc Biol. Oct 1998;18(10):1548-1553.

7. Aitman TJ, Godsland IF, Farren B, et al. Defects of insulin action on fatty acid and carbohydrate metabolism in familial combined hyperlipidemia. Arferioscler Thromb Vase Biol. Apr 1997:17(4):748754.

8. van der Kallen CI, Voors-Pette C, Bouwman FG, et al. Evidence of insulin resistant lipid metabolism in adipose tissue in familial combined hyperlipidemia, but not type 2 diabetes mellitus. Atherosclerasis. Oct 2002:164(2):337-346.

9. van der Kallen CI, Voors-Pette C, de Bruin TW. Abdominal obesity and expression of farnilial combined hyperlipidemia. Obes Res. Dec 2004;12(12):2054-2061.

10. Keulen ET, Voors-Pette C. de Bruin TW. Familial dyslipidemic hypertension syndrome: familial combined hyperlipidemia, and the role of abdominal fat mass. Am fl Hypertens. Apr 2001; 14 (4. Pt 1): $357-363$.

11. Veerkamp MJ, de Graf J, Hendriks JC, et al. Nomogram to diagnose familial combined hyperlipidemia on the basis of results of a 5-year follow-wip study. Circulation. Jun 22 2004; 109(24):2980-2085.

12. Purnell JQ, Kahn SE, Schwiarz RS, et al. Relationship of insulin sensitivity and ApoB levels to intraabdominal fat in subjects with familial combined hyperlipidemia. Anerioseter Thromb Vasc Biol. Apr 2001:21(4):567-572. 
13. Venkatesan $S$, Cullen P. Pacy $P$, al al. Stable isotopes show a direct relation between VLDL apoB overproduction and serum friglyceride levels and indicate metabolically and biochemically coherent

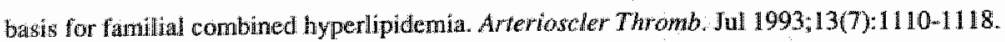

4. Verseyden C, Meijsen S. Castro Cabezas M. Postprandial changes of apoB-100 and apoB-48 in TG rich lipoproteins in farillal combined hyperlipidemia. J Lipid Res. Feb 2002;43(2):274-280.

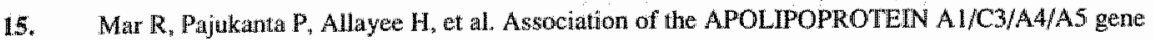
cluster with triglyceride levels and LDL particle size in familial combimed hyperlipidemia. Circ Res. Apr $16,2004,94(7): 993-999$.

16. Allayee $\mathrm{H}_{1}$ Castellani $\mathrm{LW}$, Cantor $\mathrm{RM}$, et al. Biochemical and genetic association of plasma apolipoprotein A-II levels with familial combined thyperlipidemia Circ Res. Jun 132003;92(11):12621267.

17. Rauh $\mathrm{G}$, Schuster $\mathrm{H}$, Muller $\mathrm{B}$, et al. Genevic ewidence from 7 families that the apolipoprotein $\mathrm{B}$ gene is not involved in familial combined hyperlipidemia. Aheroscterosis. Thi 1990;83(1):81-87.

18. Pajukanta $P_{*}$ Porkka KV, Antikainen M, at al. No evidence of linkage between familial combined hyperlipidemia and genes encoding lipolytic enzymes in Finnish families. Arrevioscler Thromb Vasc Biol. Mary 1997;17(5):84:1-850.

19. Shoulders CC, Jones EL, Naoumova RP. Genetics of fanilial combined hyperlipidemia and risk of coronary theart disease. Hum Mol Genet. Apro $12004 ; 13$ Spec No I R 149-160.

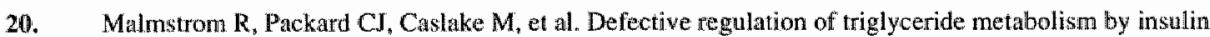
in the liver in NIDDM. Diaberolagia. Apr 1997;40(4):454-462.

21. McGarry JD, Dobbirss RL. Fatty acids, lipotoxicity and insulin secretion. Diabefologia. Feb $1999 ; 420): 128-138$.

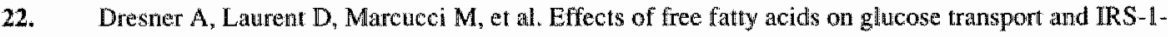
associated phosphatidylinositol 3-kinase activity. J Clin Invest. Jan 1999;103(2):253-259.

23. Kim IK. Fillmore JJ, Chen $\mathrm{X}$, et al. Tissue-specific overexpression of lipoprotein lipase causes tissuespecific ansulin resistance. Proc Nat Acd Sci U SA. Jun 192001:98(13):7522-7527.

24. Ykin-Jarvinen $H$. Ectopic fat accumulation: an important cause of insulin resistimce in humans. $J R$ Soc Med 2002,95 Suppl $42: 39-45$

25. Frayn KN. Adtipose tissue as a buffer for daily lipid flux. Diabetohogia. Sep 2002;45(9):1201-1210.

26. Murray RK GD. Mayes PA, Rodwell VW. Harper"s Biochemismy 25 th ed: Appleton \& Lange; 2000.

27. Lewis GF. Carpentier A. Adeli $K$, et al. Disordered fat storage and mobilization in the pathogenesis of insulin resistance and type 2 diabetes. Emlocr Rev. Apr 2002;23(2);201-229.

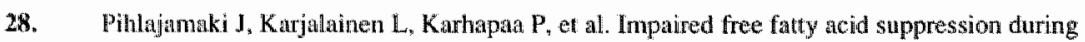
lypermsullinemia is a characteristic finding in familind combined hyperlipidemia, but insulin resilstance is observed only in hypertriglyceridenic patients. Arterioscler Thowh Vasc Biol. Jan 2000;20(1): 164170.

29. Reymisdotir S, Eriksson M, Angelin B, al. Impaired activarion of adpocyte lipolysis in familial combined hyperlipidemia. J Clan Invest. Miay 1995;95(5):2161-2169. 
30. Reymisdottir $S$, Angelin $B$, Langin $D$, et al. Adipose tissue lipopnote in lipase and homont sensitive lipase. Contrasting findings in fannhlal combined hyperlipidemand ansulin resistance symdrome. Arterioscher Thromb Vasc Biol. Oct 1997; 17(10):2287-2292.

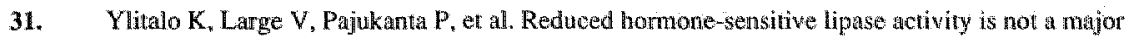
metabolic defect in Finnish FCHL familles. Atheroscleroxis. Dec 2000,153(2):373-381.

32. Carpentier A, Patterson BW, Leung $N$, et al. Sensitivity to acute insulin mediated suppression of plasna free fatty acids is mot a determinant of fasting VLDL triglyceride secretion in healthy humans. Diaberes, Jun 2002,51(6): 1867-1875.

33. Eurlings PM, Var Der Kallen CJ, Geurts JM, et al. Identification of differentally expressed genes in subcutaneous adipose tissue from subjects with familial combined hyperlipidemia. I Lipid Res. Jum $2002 ; 43(6): 930-935$.

34. Eurlings PM, van der Kallen CJ, Vermeulen VM, et al. Variants in the PPARgamma gene affect fatty acid and glycerol metabolisn in familial combined hyperlipidernia. Mol Genet Metab. Nov $2003 ; 80(3): 296-301$.

35. Verseyden $C$, Meijsen $S_{n}$ Cabezas MC. Effects of atorvastatin on fasting plasma and marginated apol upoproteins $\mathrm{B} 48$ and $\mathrm{B} 100$ in large, triglyceride-rich lipoproteins in fanilial combined hyperlipidemian d Cliw Endocrinol Metot. Oct 2004:89(10):502:-5029.

36. Verseyden $\mathrm{C}$, Meijssen $\$$, van Dijk $\mathrm{H}$, et al. Effects of atorvastatin on Irsting and postprandial complement component 3 response in familial combined hyperlipidemiat J Lipid Res. Nov $2003 ; 44(11): 2100-2108$.

37. Sniderman $A D$, Cianflone $\mathrm{K}$, Summers $\mathrm{L}$, et al. "The acylation-stimulating protein pathway and regulation of postprandial metabolism. Proc Nur Soc. Jul 1997;56(2):703-712.

38. Ylitalo $K$, Pajukanta $P$, Meri $S$, et al. Serum $C 3$ but not plasma acylation-stimulating protein is elevated in Fimish patients with familial combined hyperipidemia. Arterioscler Thromb Vasc Biol. May" $2001: 21(5): 838-843$.

39. Meex S. Upregulation of CD36 in preadipocytes in patients with tamilial cormbined hypenlipidemia. In press. 2005.

40. Bredie SI, Tack CJ, Smits P. et al. Nonobese patients will familial conbined hypenlipidemia are insulin resilstanl compared with their nonaffected relatives. Arterioscler Thromb Vousc Biol Jul $1997 ; 17(7): 1465-1471$

41. Randle PJ. Regulatory interactions between lipids and carbohydrates: the glucose fatty acid cycte after 35 years. Diaberes Metab Rew. Dec 1998; 14(4):263-283.

42. Petersen KF. Shulman GL Cellular mechuanism of insulin resistance in skeletal muscle. $J R$ Soc $M$. $d$. $2002 ; 95$ suppl $42: 8-13$.

43. Bays H, Mandarino L. DeFronzo RA. Role of the adpocyte, free fatly actds, and ectopic fat in pathogenesis of type 2 diabetes mellitus: perox isomal proliferator-activated receptor agonists provide st rational therapeutic approach. I Clin Endocrimol Mendb. Fet 2004:89(2):463-478. 
44. Zick Y. Wusulin reststance a phosphoryation-based uncoupling of insulin signaling. Trends Cell Biol. Now 2001:1 1(11):437-441.

45. Schwarz $\mathrm{M}, \operatorname{Linfoot} P$, Dawe $D$, et al Hepatic de nowo lipogenesis in norimoinsulinenic and hypierinsulnentic subjects consuming high-fat, low-carbohydrate and low-fat, high-carbohydrate iswenergetic diets. Arn I Chw Nur. Jan $2003 ; 77(1): 43-50$.

46. Pajuksuta P, Lilja HE, Sinsheimer JS, wt al. Familial combined hyperlipidemia is associated with upstrexim transcription factor 1 (USF1). Nat Genet. Apr 2004,36(4):371 *376.

47. Towle HC, Kaytor EN, Shih HM. Regulation of the expression of lipogenic enzyme genes by carbohydrate. Annw Rev Nutr. 1997, 17:405-433.

48. Towle HC. Glucose and CAMP: adversaries in the regulation of hepatic gene expression. Proc Narl Acad Sci USA. Wov $20200198(24): 13476-13478$.

49. Casado M, Vallet VS, Kahm $A$, et al. Essential role in vivo of upstream stimulatory factors for a normal dietary response of the fatty acid synthase geme in the liver.J Biol Chem. Jan 22 1999;274(4);2009. 2013.

50. Chen G, Liang G, Ou J, al Central role for liver $X$ receptor in insulin-mediated activation of Srebp1c transcription and stimulation of fatty acid symthesis in liver. Proc Narl Acad Sci USA. Aug 3 $2004 ; 101(31): 11245-11250$.

51. Horton JD, Goldstem $\Omega$, Brown MS. SREBPs: activators of the complete program of cholesterol and futy acid synthesis in the liver. $J$ Clin Invest. May 2002; 109(9);1125-1131.

52. Browming JD, Horton JD. Molecular mediators of hepatic steatosis and liwer ingury. $J$ Clin /nvest. Jul 2004; 1 14(2): 147-152.

53. Haque $M$, Sanyal AJ. The metabolic abnormalities associated with non-alcoholic fatty liver disease. Best Proct Res Clin Gastroenterol Oct 2002;16(5):709-731.

54. de Bruin TW, Georgieva AM, Browwers MC, et al. Radiological evidence of nonalcoholic fatty liver disease in fanilial combined hyperlipidemia. Am I $M$ d. Jun 15 2004;116(12):847-849.

55. Malmstrom R. Packand CJ, Watson TD, et al. Metabolic basis of hypotriglyceridemic effects of insulin in nomal men. Arferioscher Thromb Vase Biol. Jul 1997;17(7):1454-1464.

56. den Boer M, Voshol PJ, Kuipers F, et fil. Hepattc steatosis: a mediator of the metabolic syndrome. Lessons from animal models. Arrevioscler Thromb Vasc Biol. Apr 2004:24(4):644-649.

57. Adiels M, Boren J, Castake MJ, et al. Overproduction of VLDLL driven by hyperglycemia is a dominant feature of dimbetic dyslipidenial. Arterioscler Thromb Vasc Brot. Aug 2005;25(8):1697-1703.

58. Gibbons GF, Bartert SM. Sparks CE, et al. Extacellular fatty acids are not utilized directly for the synthesis of very lowndensity lipoptotein in primary cultures of rat hepatocytes. Biochem $J$. Nov 1 1992:287 ( Pr 3):749.753.

59. Malmstron R. Packad CH, Caslake M, el al. Effect of heparin-stimulated plasma lipolytic activity on VLDL APO B subclass metabolism in normal subjects. Atherosclerosis. Oct 1999; 146(2):381-390.

60. Alexander CA, Haniliton RL, Havel RI. Subcellular localization of B apoprotein of plasma lipoproteins in falliver $J$ Cell Biof. May 1976;69(2):241-263. 
61. Sheiness GS, Sellers 1A. Very-low-density lipopiotein assenbly and secretion, Curr opin Lipidol. Apr. $2001 ; 12(2): 151-157$.

62. Gibbons GF, Wiggins D. Brown AM, et al. Synthesis and function of hepatic wety-low-density lipoprotein. Brochem Soc Trans. Feb 2004:32(Pt 1):59-64.

63. Hussain MM, Shi J, Dreizen P. Microsomal triglyceride transfer protein and its nole in apob-lipoprotein assembly. I Lipid Res. Jan 2003;44(1)"22-32.

64. Pan M, Cederbaum Al, Zhang YL, et al. Lipid peroxidation and oxidan stress regulate hepatio apolipoprotein B degradation and VLDL production. I Clin Lnwert. May 2004;1 13(9):1277-11287.

65. Gusarova V, Brodsky JL, Fisher EA. Apolipoprotein B 100 exit from the endoplasmic reticulum (ER) is COPII-dependent, and its lipidation to very low density lipoprotein occurs post-ER. I Bial Chem. Nov $282003 ; 278(48): 48051-48058$.

66. Asp L, Claesson C, Boren J, et all. ADP-ribosylation factor 1 and its activation of phospholipase D ane important for the assembly of very low density lipoproteins. I Biol Chem. Aug 25 2000:275(34):2628526292.

67. Kissebah AH, Alfarsi $S_{i}$ Evans DJ. Low density lipoprotein metabolism in familial combined hyperlipidemia. Mechanism of the mulitiple lipoprotein phenotypic expression. Arteriosclerosis. Nov. Dec 1984;4(6):614-624.

68. Sato R, Miyamoto W, Inoue J, el al. Sterol regulatory element-binding protein negatively regulates microsomal triglyceride transfer protein gene transcription. $/$ Biol Chem. Aug 27 1999;274(35):24714m 24720 .

69. Samuel VT, Liu ZX, Qu X, et al. Mechamism of hepatic insulin resistance in non-alcoholic fatly liver disease. J Biot Chem. May 272004.

70. Zoltowska $M$, Ziv $E$, Delvin E, et al. Both insulin resistance and diabetes in Psammonys obesus upregulate the hepatic machinery involved in intracellular VLDL assembly. Arterioscler Thromb Vasc Biol. Jan 2004:24(1):118-123.

71. Aouizerat BE, Alayee H, Cantor RM, et al. Linkage of a candidate gene locus to familial combined hyperlipidenia: lecithintcholesterol acyltransferase on 160. Arteriosclen Throwb Vasw Biol. Nov $1999 ; 19(11): 2730-2736$

72. Williams P. Willam"s Textbook of Endocrinology. Vol 9. Philadelphna: Saunders; 1999.

73. Bemeis KK, Krauss RM. Metabolic origins and clinical significance of LDL heterogeneity, I Lipid Res: Sep 2002;43(9):1363-1379.

74. Lamarche B, Tohernof A. Moorjani S, et all, Small, dense low-density lipoprotein particles as a predictor of the risk of ischemic heart disease in men. Prospective results from the Quebec Cardovascular Study. Circhlation. Jan 7 1997;95(1):69-75.

75. St-Pierre AC, Ruel IL, Cantin B, ef al. Conaparison of various electrophoretic characteristics of LDL particles and their relationship to the risk of ischemic beart disease. Cinculation. Nov 6

$2001 ; 104(19): 2295 \% 2299$. 
76. Williams PT, Superko MR, Haskell WL at al. Smallest LDL particles are most strongly related to coronury discase progression in men. Arterioscler Thromb Vasc Biol. Feb $12003,23(2): 314-321$.

77. Guerin M, Lassell TS, Lo Goff $\mathrm{W}$, al. Action of atorwatan in combined hyperlipidemia : preferential reduction of eholesteryl ester transfer from HDL to VLDLal particles. Arterioscler Thromb Vasc Biol. Jar $2000,20(1) 189197$.

78. Bjorkegren J, Packard $\mathrm{CJ}$, Hamuster $\mathrm{A}_{\mathrm{a}}$ et al. Accumulation of large very low density lipoprotein in plasma during intratenous infusion of a chylomicron-like triglyeeride emulsion reflects competition for A common lipolytic pathway: ILipid Res: Jan 1996;37(1):76-86.

79. Austin MA, King MC, Vranizan KM, et al. Atherogenic lipoprotein phenotype. A proposed genetic marker for coronary heart disease risk. Circulation. Aug 1990;82(2):495-506.

80. Georgleva AM. wan Greeventbroek MM, Kirauss RM, ret al. Subclasses of low-density lipoprotein and very low-density lipoprotein in familial combined hyperlipidemia: relationship to multiple lipoprotein phenolype. Arterioscles Thromb Vasc Biol. Apr 2004:24(4):744-7.49.

81. Krauss RM. Atherogenicity of triglycende-rich lipoproteins. Am I Cardiol. Feb 26 1998;81(4A):13B$17 \mathrm{~B}$.

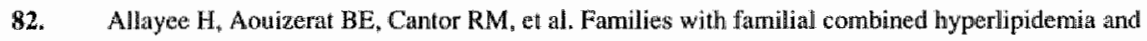
families enriched for coronary artery disease share genetic determinants for the atherogenic lipoprotein phenotype. Am I Hum Gener. Aug 1998;63(2):577-585.

83. Babirak SP, Brown BG, Brunzell JD. Famil ial combined hyperlipidemia and abnormal lipoprotein lipase. Arterioscler Thromb. Oci 1992;12(10):1176-1183.

84. Mead JR, Irwine SA, Ramji DP. L poprotein lipase: structure, function, regulation, and role in disease $f$ Mol Med. Dec 2002;80(12);753-769.

85. de Man FH, de Beer $\mathbf{E}$, van der Laarse A, wh. The hypolipidenic action of bezafibrate therapy in hypertriglyceridentia is med uated by upregulation of lipoprotein lipsse: no effects on VLDL substrate atfinity to lipolysis or LDL receptor binding. Atheroswterosis. Dec 2000; 153(2) 363-371.

86. Eurlings PM, wan der Kallen CJ. Geurts JM, et al. Identification of the PPARA locus on chromosome 22 p 13.3 ws il nodifier gene in larmilial conbirned hyperlipidemia. Mol Gener Metwo. Dec $2002 ; 77(4): 274-281$.

87. Allayee $\mathrm{H}_{4}$ Dominguez KM, Aouizerat BE, at al. Contribution of the hepatic lipase gene to the therogenic lipoprotein phenotype in familial combined byperlipidemia $/$ Lipid Res. Feb $2000,41(2), 245-252$.

88. Vakkilkinen J, Jahuanen M., Yitalo K, et al. LDL particle size in familial combined hyperlipidemia: eflects of serum lipids, lipoprotein-modifying enzymes, and lipid transfer proteins. J Lipid Res. Apr $2002 ; 4: 3(4): 598-603$.

89. van Barlingen HH, Kock LA, de Man FH, et al. In witro lipolysis of human VLDL: effect of different VLDL compositions in nomoligidemia, familial combined hyperlipidemia and familial hypertriglyceridernia. Atherosclenosis. Mar 1996,121(1):75-84. 
90. Bredie SJ, Vogelarar IM, Demacker PN, et all. Apol hoprotein E polymorphism influences lipid phenotypic expression, but not the lony density lipoprotein subfraction distribution in fanilial combined hyperlipidemia. Atheroscterosis. Oct $251996 ; 126(2): 313-324$.

91. Ericsson S, Eriksson M, Berghtund L, at. Metabolisn af plasma low density lipopnoteins in familial combined hyperlipidaemia: effect of acipimox therapy. I Intern Ned. Oot 1992:232(4):313-320.

92. Agular-Salinas CA, Hugh $P_{*}$, Barret $R$, et al. A familial combined hyperlipidernic kindred with impaired apolipoprotein B catabolism. Kinetics of apolipoprotein B during placebo and prawastatin therapy. Anterioscler Thromb Vase Biol. Jan 1997:17(1):72-82. 
Chapter 2

\section{Subclasses of Low-Density Lipoprotein and Very Low-Density Lipoprotein in Familial Combined Hyperlipidemia. Relationship to Multiple Lipoprotein Phenotype.}

A.M. Georgieva, M.M.J. van Greevenbroek, R.M. Krauss "M.C.G.J. Brouwers, V.M.M-J. Vermeulen, M.G. Robertus-Teunissen, C.J.H. van der Kallen, T.W.A. de Bruin

Arteriosclerosis, Thrombosis, and Vascular Biology 2004,24:744-9

University of Maastricht, The Netherlands

'University of Califomia, Berkeley, USA 


\section{Abstract}

The present study addressed the presence of distinct metabolic phenotypes in FCHL, in relation to mall dense LDL (sd LDL) and VLDL subclasses. Hyperlipidemic FCHL. relatives $(n=72)$ were analyzed for $L D L$ size by gradient gel electrophoresis. Pattem $B L D L$ (sd LDL, particle size $<258 \AA$ ), and pattern A LDL (buoyant LDL, particle size $\geq 258 \AA$ ) were defined. Analyses showed bimodal distribution of LDL size, associated with distinct phenotypes. Subjects with predominantly large, buoyant LDL showed a hyperchollesterolemic phenotype and the highest Apo B levels. Subjects with predominantly sd LDL showed a hypertriglyceridemic, low HDL-cholesterol phenotype, with moderately elevated Apo B, total and LDL cholesterol levels. Subjects with both buoyant LDL and sd LDL (pattem $A B, n=7$ ) showed an intermediate phenotype, with high normal plasma triglycerides. VLDL subfraction analysis showed that the sd LDL phenotype was associated with a 10-times higher number of VLDL1 particles of relatively lower Apo AI and Apo E content, as well as smaller VLDL2 particles, in combination with increased plasma insulin concentration in comparison to pattern A. The present observations underscore the importance of the VLDL-TG metabolic pathway in FCFL as an important determinant of the phenotypic heterogeneity of the disorder. 


\section{Introduction}

FCHL is a metabolic disease, delineated as a genetic disorder of lipid metabolism almost 3 decades ago'. It is associated with a two to five-fold increased risk of premature coronary artery disease (CAD) ${ }^{1.2}$. In spite of recent progress, the genetic and metabolic background of FCHL have not been elucidated in detail. Subjects with FCHL present with a complex phenotype which expression is influenced by genetic, metabolic and environmental factors $^{3-6}$. Affected FCHL relatives are viscerally obese ${ }^{2,4.7}$, hyperinsulinemic ${ }^{3}$, insulin resistant ${ }^{5,7}$ and can show a number of abnormalities in lipid metabolism: hypercholesterolemia and/or hypertriglyceridemia, elevated Apo B levels, small dense LDL (sd LDL), and decreased plasma HDL-cholesterol concentrations.

VLDL and LDL consist of distinct, physicochemically heterogenic subclasses". A practical characterization of the LDL profile divides it into two major phenotypes: patten $A$. characterized by a preponderance of large, buoyant particles, with peak particle diameter $(\mathrm{PPD}) \geq 258 \AA$, and pattern $\mathrm{B}$, chanacterized by predominance of sd LDL particles, with $\mathrm{PPD}$ $<258 \AA$. In the population, sd LDL phenotype and the concurrent metabolic abnormalities (relative hypertriglyceridemia and low HDL-cholesterol) have been designated the Atherogenic Lipoprotein Phenotype ${ }^{9}$, consistent with its association with an increased risk of $\mathrm{CAD}^{9}{ }^{10}$. Furthermore, pattern B LDL has been recognized as a feature of the metabolic syndrome ${ }^{11}$ and is characteristic for insulin resistant states, such as type $2 \mathrm{DM}^{12}$. It has been reported that presence of $s d \mathrm{LDL}$ is an inherent component of the dyslipidemia in FCHL $\mathrm{L}^{6.13-15}$ and shares genetic determinants with the expression of $\mathrm{FCHL}^{6,13,14}$.

The aim of the present study was to investigate in detail the sd LDL phenomenon in FCHL. Kinetic studies have shown a metabolic relationship between hepatic VLDL1 production and the appearance of sd LDL in plasma, ${ }^{8,16,17}$. It has been showim that FCHL subjects exhibit a higher production rate of VLDL-Apo B than controls ${ }^{18}$, but no distinction has been made so far between the VLDL1 or VLDL2 subclasses overproduced. We examined whether specific metabolic phenotypes are associated with pattem $A$ or pattern $B$ LDL in hyperlipidemic FCHL relatives, whether a relationship exists with the phenomenon of multiple lipoprotein phenotypes", and if specific VLDL subclasses are involved. Therefore, VLDLI and VLDL2 subclasses have been analyzed with regard to lipid and apolipoprotein 
composition in carriers of pattern A, B and AB LDL subspecies. This is the first study, to our knowledge, that addresses this issue in FCHL.

\section{Methods}

\section{Subjects}

Hyperlipidemic FCHL rellatives ( $\mathrm{n}=72,36$ men and 36 women) were recruited at the Lipid Clinic of the Maastricht University Hospital. FCHL families $(n=27)$ were ascertained as previously described ${ }^{4}$. Briefly, FCHL probands had a primary hyperlipidemia with varying phenotypic expression, including fasting plasma cholesterol (TC) $>6.5 \mathrm{mmol} / \mathrm{l}$ and/or fasting plasma triglyceride (TG) concentration $>2.3 \mathrm{mmol} / \mathrm{l}$, and a positive family history of premature CAD, i.e. before the age of 60 . In addition, FCHL probands had no tendon xanthomas, no Apo E2/E2 genotype, and normal thyroid-stimulating hormone concentrations. Obesity (BMI $>30 \mathrm{~kg} / \mathrm{m}^{2}$ ) or diabetes was an exclusion critterion for the ascertainment of a FCHL proband. The hyperlipidemic FCHL subjects, who were included in the present study, had been ascertained as an affected relative in a FCHL family, which contained at least one other first-degree relative with a different lipoprotein phenotype ${ }^{1}$. In the present study, 38 subjects exhibited Fredrickson Ila lipoprotein phenotype, 17 type Itb and 17 type IV. The Human Investigation Review Committee of the Academic Hospital Maastricht approved the study protocol and all subjects gave informed consent.

\section{Methods}

Subjects were studied after an overnight fast $(12-14 \mathrm{~h})$ and at least 3 days without alcohol consumption. Any lipid lowering medication was stopped for two weeks before blood samples were collected. Venous blood was collected in prencooled EDTA ( $1 \mathrm{mg} / \mathrm{mL}$ ) containing tubes; anthropometric measurements, calculation of waist-to-hip ratio (WHR) and

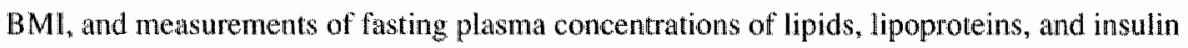
were performed as described $d^{4}$. Analyses of LDL subclass distributions, calculation of LDL. peak particle diameter, and assignment of qualitative LDL subclass pattern were done in by means of nondenaturing gradient gel electrophoresis in the Lawrence Berkeley National 
Laboratory, University of Califormin, Berkeley, USA, as described elsewhere ${ }^{8.14}$. VLDLI and VLDL2 subfractions were separated by density gradient ultracentrifugation, as described by Zhao et al. ${ }^{19}$, with minor modifications, which represent ultracentrifugation at $160000 \mathrm{~g}$ for $2.5 \mathrm{~h}$ at $4^{\circ} \mathrm{C}$ in a SW40 Ti rotor, and collection of fractions starting from the top of the tube, where the upper $1.5 \mathrm{ml}$ represents VLDL1 and the lower $5 \mathrm{ml}$ represent VLDL2. In the VLDL1 and VLDL2 subfractions, concentrations of cholesterol and triglyceride were determined in triplicate by standard laboratory techniques, and apolipoproteins (Apo) Al, All, B, CII, CIII and E in duplicate by commercial immunoassay Human Apolipoprotein Lincoplex Kit (Cat. APO-62K, Linco Research, Inc., Missouri, USA).

\section{Statistical analyses}

A $t$ test was used to analyze differences between the groups. Log transformed values of triglycerides, $\mathrm{BMI}$, insulin were used in the analyses, because these variables did not follow the normal distribution. Kolmogorov-Smirnov statistics was used to test nomality of LDL size distribution. Pearson correlation coefficient (r) was used to describe relation between plasma and VLDL subfraction triglycerides in univariate analysis. Mann-Whitney test was used in the VLDL subclass analysis because of the sample size $(n=15)$. In all analyses the statistical package SSPS 11.0 (SSPS Inc) was used. 


\section{Results}

Distribution of LDL size and associated metabolic phenotypes in hyperlipidemic FCHL subjects

The histogram of LDL particle size in hyperlipidemic FCHL subjects showed a clear, bimodal distribution ( $p=0.001$, Figure 1 ). The mean diameter of pattern A LDL was $268.1 \AA$, and the mean of pattern $B$ LDL was $250.9 \AA$. Seven subjects showed an intermediate LDL phenotype (pattern $A B$ ), with average LDL particle diameter of $261.5 \AA_{0}$ indicating the presence of large, buoyant LDL and sd LDL.

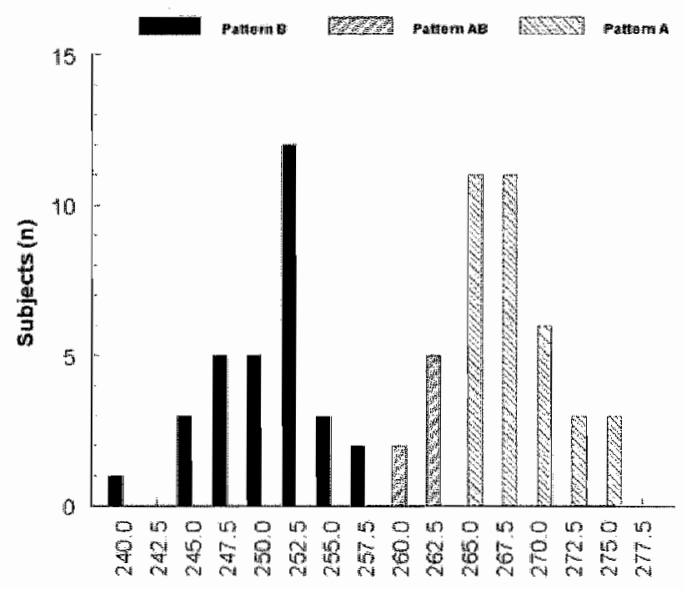

Figure 1.

Frequency distribution of LDL size LDL size (A) in hyperlipidemic FCHL relatives.

Potential differences in metabolic phenotypes between carriers of pattern A versus $B$ LDL were evaluated. "The hyperlipidemic carriers of pattern B LDL $(n=31)$ showed significantly higher triglycerides ( $\mathrm{TG}=2.8 \mathrm{mmol} / \mathrm{l}$ vs. $1.5 \mathrm{mmol} / \mathrm{l}$ in carriers of pattern $\mathrm{A}$ LDL, p<0.001), but, remarkably, significantly lower total, LDL and HDL cholesterol, Apo B and Apo Al in comparison with pattern A LDL carriers (Table 1). Of the pattem B LDL carriers, $51.6 \%$ ( 16 of 31$)$ had total cholesterol $<6.5 \mathrm{mmol} / \mathrm{l}$. Thus, pattern B LDL associated phenotype is consistent with either Fredrickson phenotypes IIb (plasma TG $>2.3 \mathrm{mmol} / \mathrm{L}$ and LDL-chol $>4.1 \mathrm{mmol} / \mathrm{L})$, or IV (TG $>2.3 \mathrm{mmol} / \mathrm{L}$ and LDL-chol $<4.1 \mathrm{mmol} / \mathrm{L}$ ). 
Furthermore, subjects with pattern B LDL showed statistically significant higher plasma insulin concentrations. By contrast, nearly all ( 30 of 34 , or $88 \%$ ) hyperlipidemic pattern $A$ LDL subjects showed plasma TC $>6.5 \mathrm{mmol} / \mathrm{i}$ in combination with nomal TG $<2.3$ mmol/ $)$, representing hypercholesterolemia per se. Thus, the patrem A associated phenotype resembles the classical Fredrickson phenotype Ila (LDL cholesterol $>4.1 \mathrm{mmol} / \mathrm{L}$ and TG $<$ $2.3 \mathrm{mmol} / \mathrm{L}$ ). Carriers of pattern AB LDL showed intermediate phenotype, which differed significantly from the pattern $B$ associated metabolic phenotype only in plasma TG. Of the pattern AB subjects, 6 exhibited Fredrickson IIa and 1 subject IIb phenotype.

Table 1. Comparison of metabolic phenotypes between subjects with pattern A, pattern $B$ or pattern AB LDL among hyperlipidemic FCHL subjects ( $n=72)$.

\section{Pattern A LDL Pattern B LDL}

No., M/F

Age, yrs

$T \mathrm{~T}, \mathrm{mmol} / \mathrm{h}$

$\mathrm{TC}_{1} \mathrm{mmol} / \mathrm{H}$

LDL-chol, $\mathrm{mmol} / /$

HDL-chol, $\mathrm{mmol} / \mathrm{h}, \mathrm{M} / \mathrm{F}$

Apo A1, g/l

Apo B, g/l

LDL PPD, А

Insulin, $\mu \mathrm{U} / \mathrm{ml}$

BMII

WHR
$34(17 / 17)$

$51.9 \pm 13.6$

$1.5 \pm 0.5$

$7.4 \pm 0.9$

$5.8 \pm 0.9$

$0.9 \pm 0.2 / 1.1 \pm 0.2$

$$
1.5 \pm 0.2
$$

$1.5 \pm 0.2$

$268.1 \pm 3.2$

$7.4 \pm 4.2$

$26.8 \pm 3.6$

$0.94 \pm 0.07$
$31(17 / 14)$

$51.5 \pm 10.0$

$2.8 \pm 0.6^{* * * *}$

$6.4 \pm 1.4^{* * *}$

$4.5 \pm 1.4$

$0.7 \pm 0.2^{*} / 0.8 \pm 0.2^{* * *}$

$1.3 \pm 0.2^{* *}$

$1.4 \pm 0.2^{*}$

$251.0 \pm 3.9^{* 4 *}$

$10.6 \pm 6.4^{*}$

$28.1 \pm 4.6$

$0.95 \pm 0.07$
Pattern AB LDL

$$
7(2 / 5)
$$

$43.0 \pm 17.7$

$1.7 \pm 0.4111$

$7.3 \pm 0.8$

$5.6 \pm 0.8$

$0.8 \pm 0.3 / 0.9 \pm 0.2$

$1.4 \pm 0.2$

$1.6 \pm 0.3$

$261.5 \div 2.114+.4$

$9.9 \pm 7.0$

$28.5 \pm 5.0$

$0.91+0.11$

\section{Values are Mearn 4 SD}

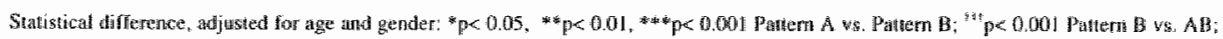

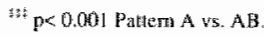

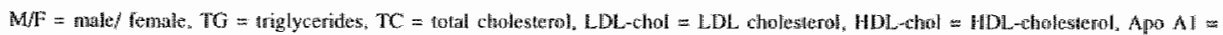

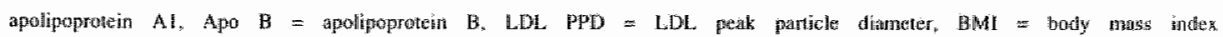




\section{Relationship between Plasma and VLDL subfraction triglycerides}

Subsequently, the lipid composition of VLDL1 and VLDL2 subclasses were analyzed in typical carriers of pattern $A(n=15)$, pattern $B(n=15)$ ar $A B$ LDL $(n=6)$.

In subjects with pattern B LDL, a statistically significant relationship was found between plasma triglycerides and VLDL1-TG $(r=.61 ; p=.015)$, but not VLDL2. Subjects with pattern $\mathrm{AB}(\mathrm{n}=6)$ ) showed a similar relationship between plasma triglycerides and VLDL $1-T G$, as observed in subjects with pattern $B$, although it did not reach statistical significance $(r=.70 ; p=.12)$. In contrast, in subjects with pattern $A L D L$, a statistically significant relationship was found between plasma triglycerides and VLDL2-TG $(r=.52, p=$. 047). The relationship with VLDLI-TG approached statistical significance( $r=47 ; p=.08)$. Therefore, the largest contribution to hypertriglyceridemia in pattern $B$ carriers (and probably in pattern AB carriers) comes from VLDL1-TG (Figure 2). In pattern A, VLDL2-TG and, to a lesser extent, VLDL1-TG contribute to plasma TG concentrations. Noteworthy, there was a statistically significant positive relationship between VLDL1-TG and plasma insulin levels in pattern $B$ subjects $(r=.64, p=01)$, but not in pattern A (Figure 3 ).

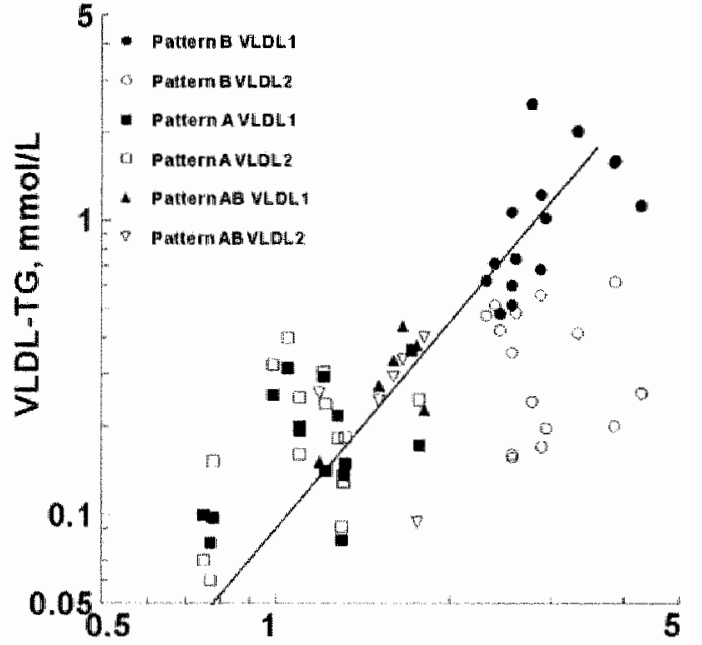

Plasma TG, $\mathrm{mmol} / \mathrm{L}$

\section{Figure 2.}

Plasma TG concentrations in all hyperlipidemic FCHL subjects were correlated with an increase in VLDL1-TG $(r=.91 ; \mathrm{p}<0.001$, regression line shown) and VLDL2-TG $(r=0.47 ; \mathrm{P}<0.01)$, which reflected increased particle number rather than particle size (as shown in Table 2). 


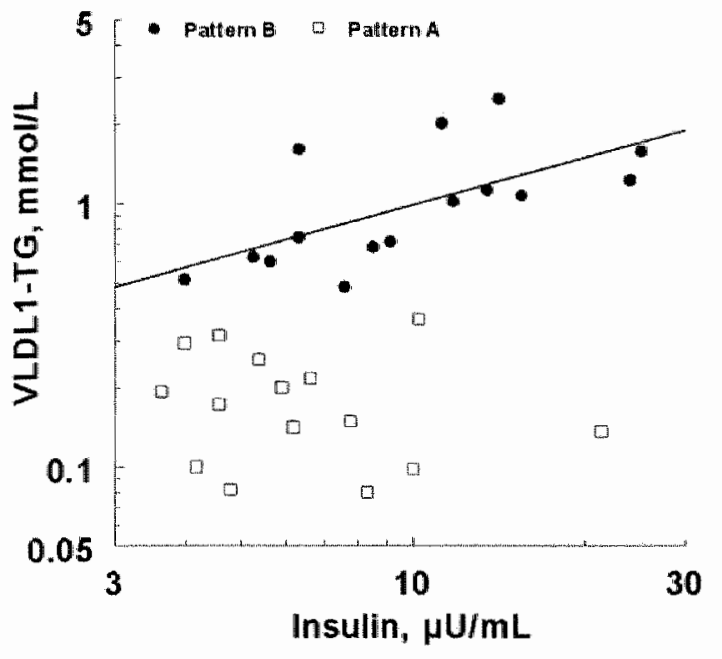

Figure 3.

There was a statistically significant positive nelationship between VLDL 1-TG and plasma insulin levels in pattern B subjects $(r=64$, $\mathrm{p}=.01$ ), but not in pattern A. Data are presented on a log scale.

\section{Lipid profile of VLDL1 and VLDL2 in patterns $A, A B$ or $B$.}

Because there is only one Apo B molecule per VLDL particle, the concentration of Apo B (nmol/L) provides information about the number of VLDL particles in plasma. Pattern $B$ subjects showed a 10 -fold higher concentration of Apo B in both VLDL subfractions than pattern A subjects (Table 2), and therefore had around 10 times more VLDL1 and VLDL2 particles in plasma, despite the fact that their mean plasma Apo B concentration was slightly lower (Table 1). Pattern AB subjects showed the most striking increase in their VLDL2 particle number, which was statistically significant when compared to pattern A subjects.

In pattern B subjects, VLDLI subfraction had a 4-fold higher TG content than the corresponding VLDL2 subfraction (Table 2). By comparison, in carriers of pattern A LDL. this ratio was 0.95 ( $\mathrm{p}<0.001)$. Also, VLDLI in pattern $B$ subjects contained $73 \%$ of all VLDL triglycerides and $65 \%$ of VLDL. cholesterol. By contrast, VLDLI in pattern A subjects contained about half of all VLDL TG, i.e $48 \%$, and only $37 \%$ of VLDL cholesterol. With regard to particle lipid composition, pattern $A$ and pattern $B$ subjects showed variations in VLDL1 particle triglyceride and cholesterol content, but on the average there was no statistical difference in lipid composition of the VLDL1 particles between the three groups. (Table 2). Therefore the higher triglyceride content of the VLDLI sublraction in pattern B 
subjects was due to a higher VLDL1 particle number, but not to the presence of triglyceridericher VLDLI (larger) particles. Interestingly, pattern A subjects seemed to have triglycerideand chollesterol-enriched VLDL2, i.e. relatively larger particles, compared to pattern $B$ and pattern $A B$ subjects. Pattern $B$ and pattem $A B$ carriers showed remarkable similarity in VLDL2 lipid content, i.e. reflecting relatively smaller VLDL2 particles.

Table 2. Lipoprotein profiles of VLDL $\mathbb{l}$ and 2 in hyperlipidemic FCHL subjects

\begin{tabular}{|c|c|c|c|c|}
\hline & Pattern A LDL. & Pattern B LDL & P Value & Pattern AB \\
\hline No. & 15 & 15 & & 6 \\
\hline \multicolumn{5}{|l|}{ VLDL1 } \\
\hline Apo Bł, nmol/L & $0.4 \pm 0.3$ & $4.6 \pm 3.8$ & 0.04 & $1.0 \pm 0.5$ \\
\hline TG per Apo B $\neq$, mmol/mg & $0.7 \pm 0.3$ & $1.2 \pm 1.6$ & NS & $0.6 \pm 0.3$ \\
\hline Total TG (mmoll) & $0.19 \pm 0.09$ & $1.10 \pm 0.59$ & $p<0.001$ & $0.30 \pm 0.10^{w k *}$ \\
\hline$\%$ of total VLDL-TG & $47.9 \pm 6.6$ & $73.1 \pm 14.3$ & & $52.6 \pm 16.1$ \\
\hline Chol per Apo B mmol/mg & $0.2 \pm 0.1$ & $0.56 \pm 0.70$ & NS & $0.30 \pm 0.50$ \\
\hline Total Cholesterol (mmol/l) & $0.06(0.03-0.08)$ & $0.43(0.22-0.67)$ & $p<0.001$ & $0.15 \pm 0.04^{k \text { knkn }}+1$ \\
\hline$\%$ of total VLDL-Chol & $37.0 \pm 12.5$ & $65.1 \pm 18.0$ & & $40.3 \pm 16.0$ \\
\hline \multicolumn{5}{|l|}{ VLDL2 } \\
\hline Apo B⿺辶, $\mathrm{nmol} / \mathrm{L}$ & $1.2 \pm 1.4$ & $11.4 \pm 1.8$ & 0.004 & $8.1 \pm 2.3+t$ \\
\hline TG per Apo B\#, mmoli/mg & $0.7 \pm 0.9$ & $0.09 \pm 0.01$ & 0.095 & $0.08 \pm 0.01 t$ \\
\hline Total TG (mmol/l) & $0.21 \pm 0.11$ & $0.35 \pm 0.16$ & 0.045 & $0.27 \pm 0.10$ \\
\hline$\%$ of total VLDL-TG & $52.1 \pm 6.6$ & $26.9 * 14.3$ & & $47.4 \pm 16.1$ \\
\hline Chol per Apo B mmol/mg & $0.3 \pm 0.1$ & $0.07 \pm 0.02$ & 0.095 & $0.07 \pm 0.01$ \\
\hline Total Cholesterol $(\mathrm{mmol} / \mathrm{l})$ & $0.11 \pm 0.10$ & $0.25 \pm 0.12$ & 0.003 & $0.25 \pm 0.11 \uparrow$ \\
\hline$\%$ of total VLDL-Chol & $63.0 \pm 12.5$ & $34.9 \pm 18.0$ & & $59.7 \pm 16.0$ \\
\hline VLDL1-TG/VLDL2-TG & $0.95 \pm 0.26$ & $4.06 \div 2.91$ & $p<0.001$ & $1.13(0.57-1.98)^{*}$ \\
\hline VLDL1-CholNLDL,2-Chol & $0.65 \pm 0.35$ & $2.20(0.95-4.67)$ & $p<0.001$ & $0.64(0.35-1.14)^{*}$ \\
\hline
\end{tabular}

Vuhes are Mean 4 SD, Median (interquatile range), VLDL-TG $=$ triglycerides in VLDL, VLDL-Chol = cholesterol in VLDL, NS = not significant. Patten Bi vs. pattern AB: " $p<0,001$ " " $p<0.01 . " p<0.05 ;$ Pattern A vs. pattem $A B:+H_{0}=0.01, t p<0.05$. Pattern $A, n=6 ;$ Pattern $B, n=6 ;$ Pattem $A B, n=5$ in these analyses. 


\section{Apolipoprotein profiles of VLDL1 and VLDL2}

Concentrations of apolipoproteins (Apo) AI, Apo All, Apo CII, Apo CIII, Apo E and Apo $B$ were measured in VLDLI and VLDL2 subfractions in representative subjects of the FCHL patients described in Table 2. Figure 4 represents the apolipoprotein content of the VLDL1 particles in pattern $A(n=6), B(n=6)$, or $A B(n=5)$ subjects, expressed as percent of total apolipoproteins (set at 100 \%) calculated per Apo B (i.e. per VLDL particle).

Noteworthy, VLDLIl particles of subjects with pattern A exhibited significantly higher Apo Al content in comparison to subjects with pattern $B$ and $A B$, and significantly higher $A p o E$ than pattern B. A similar tendency was observed for VLDL2 particles, although it did not reach statistical significance. Of note, the Apo E genotype of the subjects studied was analyzed and there was no explanation of the present results by the distribution of alleles.

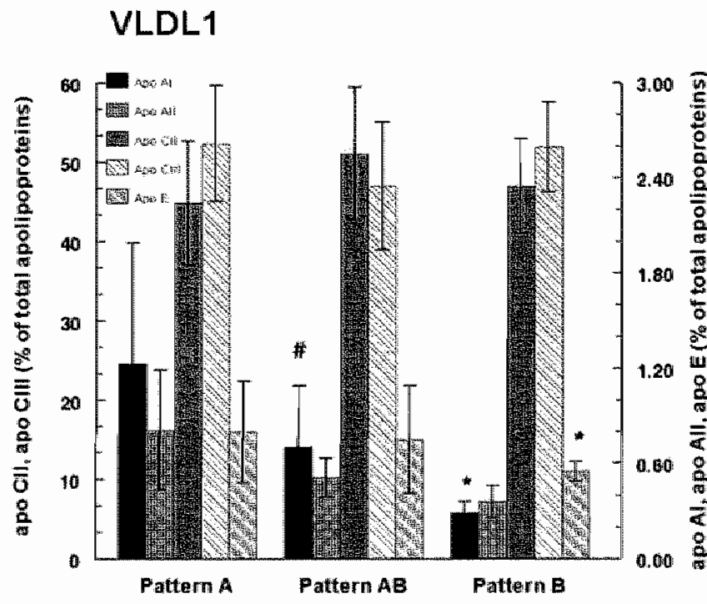

Figure 4.

Apolipoprotein profile of VLDLI particles in hyperlipidemic FCHL carriers of pattem $A, A B$ or $B L D L$ subspecies. ${ }^{*} \mathrm{P}<0.05$ pattern $\mathrm{B}$ \%s. pattern $A$. \# $P<0.05$ Pattern $A B$ vs. A. Data are expressed as percent of total apolipoproteins (mol) per Apo $B$ (mol). 


\section{Discussion}

The present findings showed that hyperlipidenic relatives from well-defined FCHL families exhibited a bimodal distribution of LDL size, in turn associated with two metabolically distinct phenotypes. Carriers of small dense LDL showed a hypertriglyceridemic, low HDL-cholesterol phenotype, quite similar to the Atherogenic Lipoprotein Phenotype, with moderately elevated plasma concentrations of Apo B, total, and LDL cholesterol. Therefore, carriers of sd LDL, also named pattern B LDL in this study, expressed hypertriglyceridemia either as Fredrickson IIb phenotype, considered classical for FCHL, or hypertriglyceridemia per se (type IV). Carriers of large and buoyant LDL particles, named pattern A LDL in this study, were characterized by a hypercholesterolemic phenotype with high Apo B and high $\mathrm{LDL}$ cholesterol in combination with near normal plasma TG concentrations. Thus, pattern A LDL is associated with Fredrickson type Ila. The existence of distinct metabolic phenotypes appears to be related to VLDL particle metabolism. This was reflected by a 10-fold higher number of VLDL1 particles, with reduced Apo AI and Apo E content, and in addition, smaller size of VLDL2 in plasma of FCHL pattern B subjects compared to pattern $A$. The present findings are consistent with the original observation of bimodality of plasma TG in FCHL and offer further insight of the pathophysiology behind the multiple lipoprotein phenotypes in FCHL.

A mechanism that is relevant to VLDL metabolism in insulin resistance ${ }^{20}$ and which appears to be important in FCHL as well is hepatic secretion of heterogeneous VLDL subspecies $^{16}$. It has been shown that FCHL subjects exhibit a 2.7 -fold overproduction ${ }^{18}$ of VLDL-Apo B. However, no distinction has been made thus far between the VLDL subclasses overproduced and their heterogeneous catabolism ${ }^{8,21}$. A novel finding in the present study is the 10-fold increased number of VLDL1 particles in pattern B subjects compared to pattern A subjects. Moreover, the VLDLL-TG content was the main contributor to the hypertriglyceridemia in pattern B FCHL subjects. In addition, pattern B subjects showed higher plasma insulin concentrations, a surrogate marker of insulin resistance, and a statistically significant positive relationship between the latter and VLDL1-TG, whereas such a relationship was not found in pattern $A$ subjects. Increased VLDL 1 secretion in insulin resistance has been formally demonstrated by stable isotope methodology in patients with 
type $2 \mathrm{DM}^{22}$. Such a kinetic study has not been performed in FCHL to date, but altogether it is plausible that a similar mechanism is operational in hypertriglyceridemic FCHL subjects as well. VLDL2 secretion, in contrast to VLDL1, is not regulated by insulin ${ }^{20}$, but is dependent; at least in part, on cholesterol availability in the liver ${ }^{*}$ VLDL2 are preferentially metabolized to large, buoyant $\mathrm{LDL}^{8}$. Accordingly, a relatively increased production rate of VLDL2-Apo $B$, or direct synthesis of LDL-ApoB ${ }^{8,16}$ can lead to the phenotypic expression of isolated. hypercholesterolemia with normal LDL size in pattern A subjects. Therefore, we suggest that differences in liver insulin sensitivity in FCHL cause differences in secretion of VLDLI and VLDL2 that can explain, at least in part, the present findings. Moreover, a similar mechanism can undertie the change of lipid phenotype observed in FCHL subjects ${ }^{23,24}$.

The present observations underscore the biological importance of the VLDL-TG metabolic pathway in FCHL, especially when put in the perspective of reported linkage and association studies. In FCHL, linkage and assaciation have been described with several genes encoding for apolipoproteins that are part of VLDL lipoproteins: the Apo AI-CHII-AIV-AV gene cluster, Apo All gene, and plasma concentrations of Apo CIII ${ }^{6,25}$, Apo All ${ }^{26}$ and Apo $B^{27}$. Moreover, linkage between Apo AI-CIII-AIV-AV gene cluster and the presence of sd LDL has been reported ${ }^{6}$. The effect of different apolipoproteins on VLDL catabolism is well known. For instance, Apo $\mathrm{E}$ affects the hepatic uptake of VLDL subfractions; Apo $\mathbb{E}_{4}$ Apo CII and Apo CIII affect lipolysis, and finally, VLDL apolipoproteins modulate lipid and protein exchange with other lipoproteins. In the present study, Apo AI and Apo E were less abundlant on VLDL particles in pattern B FCHL subjects (Figure 4) than in pattern A. Therefore, oun" data suggest that changes in VLDL apolipoprotein content may be relevant for VLDL metabolism by lipolysis and hepatic clearance, which can contribute to the accumulation of VLDL. (and VLDL2) lipoproteins observed in plasma in FCHL. subjects with sd LDL. Furthermore, it has been shown that accumulation of VLDLI in plasma, and modifier genes that affect VLDL1 catabolism, such as CETP, hepatic lipase and lipoprotein lipase, affect sd LDL frequency in FCHL $6,8,14,28,29$.

The cross-sectional design of this study prevents a definitive conclusion on the metabolical pathways involved in the phenotypic expression of FCHL. It is worth mentioning, however, that our present findings are consistent with a recent publication by Ayyobi et. al ${ }^{30}$, which associates the difference in lipoprotein phenotypes in FCHL with changes in VLDL 
and large, buoyant LDL levels. This study ${ }^{30}$ and the present abservations reflect long-term adaptation changes in FCHL and are therefore difficult to interpret in a simple manner. Further insight in the relative contribution of VLDL subclasses secretion and catabolism to the FCHL phenotype will require stable isotope studies.

In summary, a nowel finding in the present study is that hyperlipidemic FCHL subjects showed bimodal distribution of LDL size, and each peak of LDL subclasses corresponded to a distinet phenotype. Subjects with predominance of large buoyant LDL showed a hypercholesterolemic phenotype (Fredrickson type IIa), and the highest Apo B levels. Subjects with predominance of sd LDL presented with a hypertriglyceridemic, low HDLcholesterol phenotype moderately elevated Apo B levels, total and LDL cholesterol (type IIb and $(V)$, and in addition, were characterized by 10 -times higher number of VLDL particles of lower Apo AI and Apo E content (VLDL1) and smaller size (VLDL2) in plasma, compared to pattern A. The present observations underscore the importance of the VLDL-TG metabolic pathway in FCHL as an important determinant of the phenotypic heterogeneity of the disorder.

\section{Acknowledgements}

We thank E.T.P. Keulen and J. van Lin for the recruitment of FCHL patients; P.M.H. Eurlings for discussions, and S.J.R. Meex for statistical adwice. A.M. Georgiewa was supported by a Marie Curie Fellowship of the European Community program 'Quality of Life and Management of Liwing Resources" under contract number QLK5-CT-2000-60007. T.W.A. de Bruin was supported by al grant of the Netherlands Organization for Seientific Research (no. 900-95-297). LDL particle size analysis was supported by HL-18574 (R.M. Krauss, principal investigator). This reseanch was also supported by CARIM, Cardiovascular Research Institute Maastricht and the Academic Hospital Matastricht. 


\section{References}

1. Goldstein JL, Schrott HG, Hazzard WR, et al. Hyperlipidemia in coronary heart disease. II. Genetic analysis of lipid levels in 176 fannilies and delineation of a new inherited disorder "combined hyperlipidemia. J Clin Invest. Jul 1973;52(7):1544-1568.

2. Voors-Pette $C_{4}$ de Bruin TW. Excess coronary heart disease in Familial Conbined Hyperlipidemia, in relation to genetic factors and central abesity. Atherosalerosis. Aug 2001;157(2):481-489.

3. Ascaso JF, Sales J, Merchante A, et al. Influence of obesity on plasma lipoproteins, glycaemia and insulinaemia in patients with familial combined hyperlipidaemia. In J Obes Relat Metab Disord. May $1997 ; 21(5): 360-366$.

4. Keulen ET, Voors-Pette C, de Bruin TW. Familial dyslipidemic hypertension syndrome: famillial conbined hyperlipidemia, and the role of abdominal fat mass. Am J Hypertens. Apr 2001;14(4 Pt 1):357-363.

5. van der Kallen $C$, Voors-Pette $C$, Bouwman $F_{2}$ et al. Evidence of insulin resistant lipid metabolism in adipose tissue in familial combined hyperlipidemia, but not type 2 diabetes mellitus. Atheroscleroxis. Oct 2002;164(2):337.

6. Allayee $\mathrm{H}_{2}$ Aouizerat $\mathrm{BE}$, Cantor RM, et al. Families with familial combined hyperlipidemia and families enriched for coronary artery disease share genetic determinants for the atherogenic lipoprotein phenotype. Am J Hum Genet. Aug 1998;63(2):577-585.

7. Purnell JQ, Kahn SE, Schwartz RS, ef al. Relationship of insulin sensitivity and ApoB levels to intraabdominal fat in subjects with farnilial combined thyperlipidemia. Arterioscler Thromb Vasc Biol. Apr $2001: 21(4): 567-572$.

8. Berneis KK, Krauss RM. Metabolic origins and clinical significance of LDL heterogeneity. $J$ Lipid Res. Sep 2002;43(9):1363-1379.

9. Austin MA, King MC, Vranizan KM, el al, Atherogenic lipoprotein phenotype A proposed genetic marker for coronary heart disease risk. Circulation. Aug 1990;82(2):495-506.

10. Lamarche B, Tchernof A, Moorjani S, et al. Small, dense low-densily lipoprotein particles as a predictor of the risk of ischemic heart disease in men. Prospective results from the Quebec Cardiovascular Study. Circulation. Jan 7 1997:95(1):69-75.

11. Reaven G. Metabolic syndrone: pathophysiology and implications for natnagement of cardiovascular disease. Circulation. Jul 16 2002;1066(3):286-288.

12. Snidenan AD, Scantlebury T, Cianflone K. Hypertriglyceridenic hyperapob: the unappreciated atherogenic dyslipoproteinemia in type 2 diabetes mellitus. Anm Imern Med. Sep 18 2001:135(6):447. 459 .

13. Austin MA, Brunzell JD, Fitch WL, et al. Inheritance of low density lipoprotein subclass patterns in familial combined hyperlipidemia. Arterio.scherosis. Jul-Aug 1990;10(4):520-530. 


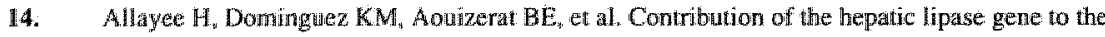
atherogenic lipoprotein phenotype in familial combined hyperlipidemia. I Lojpid Res. Feb $2000 \div 41(2): 245-252$

15. Hokanson JE, Krauss RM, Allbers II et at. LDL physical and chemical propenties in familal combined Hyperlipidemia. Arterioscler Throwb Wasc Bial. Apr $1995 ; 15(4) 452-459$.

16. Packand CJ, shepherd J. Lipoprotein heterogeneity and apolipoprotein B metabolism. Anterioscler Throwb Wasc Biol. Det 1997;17(12):3542-3556.

17. Millar J5, Packard CI. Heterogene ity of apolipoprotein B-100-containing lipoproteins: what we have leami from kinetic studies. Curr Opik Lipidol Jun 1998;9(3):197-202.

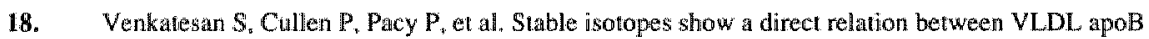
overproduction and serum triglyceride levels and indicate a metabolically and biochenically coherent busis for fam

19. Zha SP, Bastiaanse EM, Hau MF, et all. Separation of VLDL subfractions by density gradient ulltracentrifugation ILAb Clin Med. May 1995;125(5):641-649.

20. Malmstrom $R$, Packiard $C J$, Casiake $M$, et al. Effects of insulin and acipimox on VLDLI and VLDL2 apolippoprotein B production in normal subjects. Diaberes. May 1998,47(5):779-787.

21. Aguilar-Salinas CA, Hugh P, Barrett $R_{4}$ et al. A familial combined hyperlipidemic kindred with umpraired apolipoprotein B catabolism. Kinetics of apolipoprotein B during placebo and pravastatin thempy. Arterioscler Thromb Vasc Biol. Jan 1997;17(1):72-82.

22. Malmstrom R, Packand CI, Caslake $\mathrm{M}$, al Defective regulation of triglyceride metabolism by insulin in the liver in NIDDM. Diaberologia. Apr 1997:40(4):454-462.

23. Kissebah AH, Alfarsi S, Evans DJ. Low density lipoprote in metabolism in familial combined hyperlipidemia. Mechanism of the multiple lipoprote in phenotypic expression. Arteriosclerosis. NovDec $1984: 4(6): 614 \cdot 624$.

24. Brumell JD, Albers JJ, Chail A, ex al.. Plasma lipoprotens in familial combined fyperlipidemia and monogenic Tamilial bypertriglyceridemia. J Lipid Res. Feb 1983"24(2), 147-155.

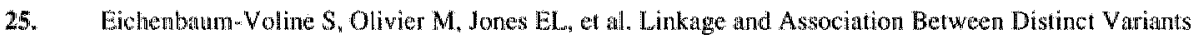
of the APOA 1/C3/A/4/A5 Gene Chster and Fanilial Combined Hyperlipidemia. Arterioscler Thomb Vase biol Od 92003 .

26. Allayee $\mathrm{H}_{n}$ Castallani LW, Cantor RM et al. Biochenical and genetic association of plasma apolipoprotein A-II levels with furnilial combined hyperlipidemia. Cre Res. Jun $132003 ; 92(11): 1262$. 1267.

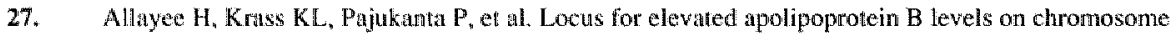

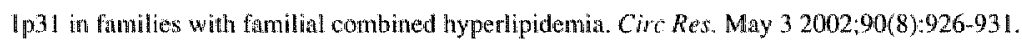

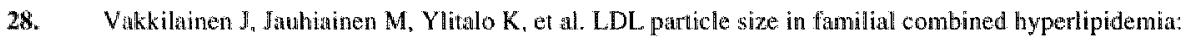
effects of serum lipids, lipoprotein-modifying enzymes, and lipid transfer protens. $J$ Lipid Res. Apr $2002: 43(4): 598-603$. 
29. Talmud PJ, Edwards KL, Tumer CM, et al. Linkage of the cholesteryl ester turasfer protein (CETP) gene to LDL particle size: use of a nowel tetranucleotide repeat within the CETP pronoter. Circulation. May $302000 ; 101121) 2461-2466$.

30. Ayyobi AF, McGladdery SH, MoNeely MJ, er al. Small dense LDL and elevated apolipoprotein B are the common characteristics for the three najor lipid phenotypes of familial combined hyperlipidemia. Arterioscler Thromb Vasc Brol Jul $12003 ; 23(7): 1289-1294$. 
Chapter 3

\section{Radiological Evidence of Nonalcoholic Fatty Liver Disease in Familial Combined Hyperlipidemia.}

Tjerk W.A. de Bruin, Anna M. Georgieva, Martijn C.G.J. Brouwers, Martijn V. Heitink,

Carla J.H. van der Kallen, Marleen M.J. van Greevenbroek

American Journal of Medicine 2004, 116:847-9 
Chapter 3 


\section{Introduction}

Familial combined hyperlipidemia is a genetic dyslipidemia (Mendelian Inheritance in Man, MIM 144250) associated with hepatic lipoprotein overproduction, insulin resistance and increased plasma concentrations of free fatty acids'. Similar alterations in metabolism are known to contribute to nonalcoholic fatty liver disease ${ }^{2}$. Nonalcoholic fatty liver disease comprises both nonalcoholic fatty liver and nonalcoholic steatohepatitis. The definitive diagnosis of the different stages of nonalcoholic fatty liver disease requires a biopsy, but ultrasonography is regarded as a highly sensitive $(89 \%)$ and specific $(93 \%)$ method to assess steatosis ${ }^{3}$. The present study evaluated the prevalence of nonalcoholic fatty liver disease in familial combined hyperlipidemia.

\section{Methods}

We evaluated seventeen patients with familial combined hyperlipidemia by upper abdomen ultrasonography, between July 2000 and July 2002. These patients were consecutively referred to the Lipid Clinic of the Mastricht University Hospital, and therefore unselected. Each patient represented a separate familial combined hyperlipidemia family. Diagnostic characteristics of familial combined hyperlipidemia families are premature myocardial infarction (before age 60), and a combination of multiple fredrickson phenotypes (IIb, IIa, or IV) among first-degree relatives. The patients with familial combined hyperlipidemia, included in this study, had a premature myocardial infarction, or a family history of such a premature myocardial infarction in a parent, uncle or atut. In addition, these patients showed a primary hyperlipidemia, usually Fredrickson type IIb and IV. Cut-off points are plasma cholesterol $>6.5 \mathrm{mmol} / \mathrm{L}(250 \mathrm{mg} / \mathrm{dL})$ and $\mathrm{TG}>2.3 \mathrm{mmol} / \mathrm{L}(200 \mathrm{mg} / \mathrm{dL})$. Known causes of nonalcoholic fatty liwer disease such as morbid obesily, type 2 diabetes, viral and toxic hepatitis, and ingestion of more than 2 alcoholic beverages per day $(20 \mathrm{~g}$ of alcohol) were excluded ${ }^{2}$. Sixteen of these seventeen patients were receiving statins; one patient without steatosis was treated with a ribrate. The lipid-lowering medication was not discontinued during the study. The control patients $(n=792)$ represented an unselected sample 
of consecutive patients who presented for upper abdominal ultrasonography in the period January 2001 to March 2001 at the University Hospital Maastricht, excluding patients with familial combined hyperlipidemia. No other clinical data was available. The study was performed with the approval of the Human Investigation Review Committee of the Academic Hospital Maastricht.

\section{Ultrasound}

Ultrasonography of the liver was done at the Department of Radiology using an ATL HDI 5000 system (Bothell, Washington, USA) with a C 5-2 transducer by independent radiologists. Conventional criteria for nonalcoholic fatty liver disease are increased echogenicity of liver compared to kidney parenchyma, decreased visualization of intrahepatic blood vessels, and posterior beam attenuation ${ }^{3}$.

\section{Measurements}

Anthropometric measurements and measurements of fasting plasma cholesterol, triglycerides and apolipoprotein B were done as described ${ }^{4}$. Serum alanine and aspartate aminotransferase, and gamma-glutamyl transferase levels were assessed in the Clinical Chemistry Laboratory of the Maastricht University Hospital.

\section{Statistical analyses}

Mann-Whitney nonparametric test was used to analyze differences between the groups in Table 1, and Fisher"s exact test to compare the prevalence of nonalcoholic fatty liver disease between familial combined hyperlipidemia patients and the control sample. Statistical analyses were performed using the Statistical Package for Social Sciences software (SSPS 11.0 for Windows, SSPS Inc, Chicago, Illinois).

\section{Results}

Nonalcoholic fatty liver disease was found in $76 \%(13$ of 17$)$ of patients with familial combined hyperlipidemia ( $95 \% \mathrm{Cl}: 56 \%$ to $96 \%$ ). By contrast, in the control sample, $10 \%$ (80 
of $792 ; 95 \% \mathrm{CI}: 8 \%$ to $12 \%$ ) were diagnosed with nonalcoholic faty liver disease $(\mathrm{P}<$ 0.001 ). Notably, $92 \%$ (12 of 13 ) of the patients with familial combined hyperlipidemia and nonalcoholic fatty liver disease exhibited hypertriglyceridemia (Table 1), either as combined hyperlipidemia (Fredrickson type IIb; $\mathrm{n}=6$ ), or as isolated hypertriglyceridemia (Fredrickson type IV; $n=6$ ).

Table 1. Characteristics of FCHL patients with or without radiological evidence of nonalcoholic fatty liver disease.

\begin{tabular}{|c|c|c|c|}
\hline & $\begin{array}{l}\text { Steatosis present } \\
\qquad(\mathrm{n}=13)\end{array}$ & $\begin{array}{l}\text { Steatosis absent } \\
\qquad(n=4)\end{array}$ & P Value \\
\hline Male sex & $10(77)$ & $2(50)$ & \\
\hline Age, yrs & $51 \pm 11$ & $65 \pm 10$ & 0.01 \\
\hline \multicolumn{4}{|l|}{ FCHL markers: } \\
\hline Waist-to-hip ratio & $0.94 \pm 0.06$ & $0.94 \pm 0.06$ & 0.95 \\
\hline Body mass index, $\mathrm{kg} / \mathrm{m}^{2}$ & $27 \pm 3$ & $26 \pm 4$ & 0.35 \\
\hline Total cholesterol, $\mathrm{mmol} / \mathrm{L}^{*}$ & $6.5 \pm 1.5$ & $5.5 \pm 0.9$ & 0.20 \\
\hline Triglycerides, $\mathrm{mmol} / \mathrm{L}+$ & $3.3(2.6-7.0)$ & $1.6 \pm 0.5$ & 0.002 \\
\hline Apolipoproteim B, g/L & $1.3 \pm 0.3$ & $1.1 \pm 0.2$ & 0.91 \\
\hline \multicolumn{4}{|l|}{ Liver biochemistry tests: } \\
\hline Aspartate aminotransferase, $\mathrm{U} / \mathrm{L}$ & $24 \pm 7$ & $29 \pm 9$ & 0.10 \\
\hline Alanine aminotransferase, $\mathrm{U} / \mathrm{L}$ & $35 \pm 15$ & $23 \pm 11$ & 0.32 \\
\hline Gamma glutamyltransferase, $U / L$ & $37 \pm 25$ & $29 \pm 22$ & 0.96 \\
\hline
\end{tabular}

Vahes are number (\%), mean $\pm \mathrm{SD}$, or median (range).

*For $\mathrm{mg} / \mathrm{dL}$ divide by $0,026,+$ For $\mathrm{mg} / \mathrm{dL}$ divide by 0.011 .

One of these 13 subjects exhibited a hypercholesterolemic phenotype (type Ila) with high normal plasma triglycerides of $1.9 \mathrm{mmol} / \mathrm{L}$. Concentrations of serum aminotransferases and gamma glutamyltransferase were not different between familial combined hyperlipidemia subjects with nonalcoholic fatry liver disease and those without. Four subjects with steatosis presented with slightly elevated alanine aminotransferase ( $>40 \mathrm{U} / \mathrm{L}$ ), probably reflecting the 
presence of nonalcoholic steatohepatitis. However, ultrasonograplay cannot make a distinction between nonalcoholic steatohepatitis and nonalcoholic fatty liver.

\section{Discussion}

This report established that nonalcoholic fatty liwer disease was present in a considerable fraction ( $95 \% \mathrm{Cl}: 56 \%$ to $96 \%$ ) of unselected subjects with familial combined hyperlipidemia and, specifically, in each of the hypertriglyceridemic subjects. The reported prevalence of nonalcoholic fatty liver disease in the general population varies between $10 \%$ and $24 \%{ }^{2}$, sinnilar to the frequency of $10 \%(95 \% \mathrm{Cl}: 8 \%$ to $12 \%)$ in our control patient sample. Given an estimated population frequency of up to $2.0 \%$ for familial combined hyperlipidemias, it can be concluded that it represents a quantitatively important contribution to the prevalence of nonalcoholic fatty liver disease, potentially representing one in five to ten cases of nonalcoholic fatty liver clisease in the population.

Familial combined hyperlipidemia is characterized by excess supply of free fatty acids, lipoproteins and lipoprotein remnants to the liver ${ }^{6}$, most likely a consequence of insulin resistance and impaired adipose tissue activity in the postprandial state ${ }^{\prime}$. Therefore, nonalcoholic fatty liver disease may reflect lipid re-distribution, where fatty acids are deposited over time as triglycerides in liver and visceral fat ${ }^{4.7}$. In addition, genetic mechanisms specific for familial combined hypertipidemia can contribute to abnormal hepatic lipid metabolism and steatosis. We recently identified Peroxisome-Proliferator-Activated Receptor-alpha and-gamma genes as modifier genes in familial combined hyperlipidemia ${ }^{8.9}$. Peroxisome-Proliferator-Activated Receptor-alpha as well as -gamma activity can affect hepatic lipid content, as shown recently ${ }^{10,11}$. The presence of steatosis in subjects with familial combined hyperlipidemia can contribute to increased production of triglyceride carrying very low-density lipoproteins, which results in hypertriglyceridemia. Elevated plasma triglyceride levels were found in $92 \%$ of the patients with familial combined hyperlipidemia and nonalcoholic fatty liver disease. Thus, the present observation suggests a direct relationship between steatosis and hepatic lipoprotein overproduction in familial combined llyperlipidemia. This finding is in line with previous publications that 
hypertriglyceridemia is a common finding in patients with nonalcoholic fatty liver disease and insulin resistance ${ }^{2,12}$.

In summary, there is a high prewalence of nonalcoholic fatty liver disease in familial combined hyperlipidemia, which suggests that familial combined hyperlipidemia potentially underlies one in five to ten population cases with nonalcoholic fatty liver disease. The present data suggest that hepatic steatosis is part of familial combined hyperlipidemia, specifically the hypertriglyceridemic phenotype. Further studies may provide insight in the mechanisms leading to nonalcoholic fatty liver disease in familial combined hyperlipidemia, and contribute to measures to prevent steatosis.

\section{Acknowledgements}

T.W.A. de Bruin, MD, PuD, has been supported by a grant of the Netherlands Organization for Scientific Research (no. 900-95-297). A.M.Georgiewa, MD has been supported by a Marie Curie Fellowship of the European Community program 'Quality of Life and Management of Living Resources' under contract number QLK5-CT-2000-60007, and a Job Cohen Grant of the Maastricht University. 


\section{References}

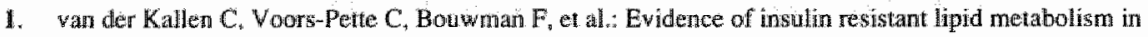
aclipose tissue in familial combined byperlipidemia, but not type 2 diabetes mellitus. Atherosclerosis 2002; 164: $337-46$.

2. Angulo P: Nonalcoholic farty liver disease. $\mathbb{N}$ Fingl J Med 2002; 346: 1221-31.

3. Joseph AE, Sawerymurtu SH, al-Sam S et al.: Comparison of liwer histology with ultrasonography in alssessing diffuse parenchymal liver disease. Clin Radiol 1991; 43:26-31.

4. Keulen ET, Voors-Pette C, de Bruin TWA: Familial dyslipidemic hypertension syndrome: familial combined hyperlipidemia, and the role of abdominal fat mass. Am J Hypertens 2001; 14: $357-63$.

5. Grundy SM CA, Brunzell JD: Familial Combined Hyperlipidemia Workshop. Arteriosclerosis 1987; 7: 203207.

6. Verseyden $C_{v}$ Meijssen $S$, Castro Cabezas M: Postprandial changes of apoB-100 and apoB-48 in TG rich lipoproteins in familial combined hyperipidemia. J Lipid Res 2002; 43: 274-80.

7. Lewis GF, Carpentier A, Adeli K, Giacca A: Disordered fat storage and mobilization in the pathogenesis of insulin nesistance and type 2 diabetes. Endocr Rev 2002; 23:201-29.

8. Eurlings PM, van der Kallen CJ, de Bruin TWA et al.: Identification of the PPARA locus on chromosone 22q13.3 as a modifier gene an familial combined hyperlipidernia Mol Genet Metab 2002; 77: 274-81.

9. Eurlings PM, yan der Kallen Cf, Vermeulen VMM-J, de Bruin TWA: Variants in the PPARY gene affect fatty acid and glycerol metabolism in familial combined hyperlipidemia. Mol Genet Metab 2003; 77: 296301.

10. Sugden MC, Bulner K, Gubbons GF, et al.: Peroxisome-proliferator-activated receptor-alpha (PPARalpha) deficiency leads to dysregulation of hepatic lipid and carbohydrate metabolism by fatty acids and insulin. Binchem I 2002: 364: 361.R.

11. Neuschwander-Tetri BA, Brunt EM, Bacon BR, et al.: Improved nonalcoholic steatohepatitis after 48 weeks of treatment with the PPAR-gamma ligand rosigtitazone. Hepatology 2003; 38:1008-17.

12. Haque M, Stanyal AJ: The metabolic abnomalities assaciated with nonalcoholic fatty liwer disease. Best Pract Res Clin Gastroenterol 2002; 16: 709-31. 
Prothrombotic Markers in Familial Combined Hyperlipidemia. Evidence of Endothelial Cell Activation and Relation to Metabolic Syndrome.

Anna M. Georgieva', Hugo ten Cate ${ }^{2}$, Eric T. P. Keulen', René van Oerle²; José W.P. GoversRiemslag ${ }^{2}$, Karly Hamulyák ${ }^{2}$, Carla J.H. van der Kallen ${ }^{1}$, Marleen M.J. van Greevenbroek", Tjerk W.A. de Bruin'

Atherosclerosis $2004,175: 345-51$

Laboratory of Molecular Metabolism and Endocrinology ${ }^{\prime}$

Laboratory of Clinical Thrombosis and Hemostasis ${ }^{2}$

Department of Internal Medicine, University of Maastricht 


\section{Abstract}

Familial combined hyperlipidemia is characterized by a varied combination of hypertiglyceridemia and hypercholesterolemia within a family, and a high risk of premature coronary artery disease. The present study evaluated a number of potential prothrombotic markers in familial combined hyperlipidemia, and studied their relationship to the hypercholesterolemic (Fredrickson type Ila) and hypertriglyceridemic (IIb and IV) phenotypes. Selected prothrombotic markers were studied in 68 subjects: 34 hyperlipidemic subjects with familial combined hyperlipidemia and 34 controls. FCHL patients exhibited significantly higher Thrombin-Antithrombin complex (TAT), activated coagulation factor XII (F XHa), von Willebrand Factor ( $\mathrm{WWF}$ ), Plasminogen Activator Inhibitor-1 (PAI-1) and tissue derived Plasminogen Activator ( $t$-PA) values in comparison to controls. Within the subgroup of familial combined hyperlipidemia subjects, elevated PAI 1 activity and soluble Thrombomodulin levels were particularly associated with features of the metabolic syndrome, including hyperinsulinemia, hypertriglyceridemia, and predominance of small dense LDL.

A general pattem of activated blood coagulation and endothelial activation is present in all hyperlipidemic subjects studied, independent of metabolic phenotype. In those familial combined hyperlipidemia subjects with features of the metabolic syndrome, impaired fibrinolysis can provide an additional cardiovascular risk factor. 


\section{Introduction}

Familial Combined Hyperlipidemia (FCHL) is a genetic metabolic disorder, associated with a two to five-fold increased risk of premature coronary artery disease $\mathrm{e}^{\mathrm{l}-3}$. Characteristic for FCHL is a primary hyperlipidemia with varied phenotypic expression (Fredrickson type IIa, IIb or IV) among FCHL family members ${ }^{1}$. In addition, FCHL subjects can show features of the metabolic syndrome: elevated plasma insulin concentrations in the presence of insulin resistance, visceral obesity, hypertension, decreased plasma HDL concentrations and predominance of $\mathrm{sd} \mathrm{LDL}^{2,4}$.

Systemic blood coagulation activity contributes importantly to acute coronary events ${ }^{5,}$ ${ }^{6}$. Data from several studies suggest that markers of activated blood coagulation (TAT, factor XIIa, D-dimers), impaired fibrinolysis (PAI-1, t-PA) or endothelial activation (vWF, sTM) have a role as indicators of cardiovascular disease, and predict an increased risk of acute events, especially below 60 years of age $\mathrm{e}^{7-11}$. Furthermore, heterozygous hemophilia carriers show a mild decrease in coagulation activity, but a reduction by $36 \%$ of deaths from $\mathrm{CAD}^{12}$. Systemic hypercoagulability ${ }^{5,6}$ or endothelial abnormalities ${ }^{13}$ may contribute to the higher incidence of coronary artery disease in FCHL as well. The aim of this study was to explore the activity profile of the blood coagulation system, in combination with fibrinolytic markers in patients with FCHL. For this purpose we examined, in a cross-sectional way, typical markers of coagulation and fibrinolysis activity, coupled to markers of endothelial cell activation. Some of these markers (PAI-1, TAT, vWF), have been previously associated with specific lipoprotein phenotypes ${ }^{14-16}$. Here we investigated whether the multiple lipoprotein phenotypes in FCHL (Fredrickson type lla, IIb and IV) share a common procoagulant profile.

\section{Materials and Methods}

In the framework of genetic and metabolic studies 34 hyperlipidemic FCHL subjects and 34 healthy spouses (controls) were recruited at the Lipid Clinic of the Maastricht University Hospital. The FCHL subjects represented 16 FCHL families, ascertained as previously described ${ }^{17}$. Briefly, FCHL subjects had: 1) primary hyperlipidemia with varying 
phenotypic expression, including (untreated) fasting plasma cholesterol $>6.5 \mathrm{mmol} / \mathrm{l}$ and/or fasting plasma triglyceride concentration $>2.3 \mathrm{mmol} / \mathrm{s} ; 2$ ) they were known with premature $\mathrm{CAD}$ (before the age of 60 ), or had a positive family history of $\mathrm{CAD} ; 3$ ) had at least one other first-degree relative with a different lipoprotein phenotype. Exclusion criteria for diagnosing FCHL in a family were diabetes mellitus type 2, causes of secondary lipidemia (nephrotic syndrome, hypothyroidism, morbid obesity), E2/E2 genotype and tendon xanthomas of the proband. The Human Investigation Review Committee of the Academic Hospital Maastricht approved the study protocol. All subjects gave informed consent.

Subjects were studied in the moming (8.00-11.00h) after an overnight fast (12-14 h) and at least 3 days without alcohol consumption. Any lipid-lowering medication was stopped for two weeks before blood samples were collected. Venous blood was collected in precooled EDTA ( $1 \mathrm{mg} / \mathrm{mL}$ ) containing tubes for measurement of plasma lipids and insulin, and in $3.8 \%$ sodium citrate tubes (dilution 1:10), with limited occlusion of the arm by a tourniquet, for measurements of coagulation proteins TAT, vWF, XIla, D-dimers, TF, PAI-1 and sTM. Blood collected in Biopool. stabilyte $^{\text {TM }}$ tubes was used to measure t-PA. The concentration of the TAT complex was measured by commercial ELISA techniques (Enzygnost ${ }^{\text {(i) }}$ Behring Germany); Free factor XIla by F XIIa ELISA kit, Shield Diagnostics, Ltd, UK; soluble Tissue Factor by Imubind ${ }^{*}$ TFELISA kit, American Diagnostica Inc.; PAI-1 activity was measured by Spectrolyse $(\mathrm{pl}$ ), and t-PA by TintElize / Biopool, Kordia, NL; D Dimers by VIDAS D-Dimer ${ }^{\text {, }}$, Biomerieux, USA; sTM by Trombomodulin Elisa ${ }^{\circledR}$, Roche Diagnostics, Switzerland. wWf was measured by a home made ELISA, where Dakopatt capture and conjugated rabbit anti-human $v$ Wf polyclonal antibodies were used (Dakopatt Denmark).

Anthropometric measurements, calculation of WHR and BMI, and measurements of blood pressure, plasma concentrations of lipids, lipoproteins and insulin were performed exactly as described ${ }^{17}$. Analyses of LDL subclass distributions, and calculation of LDL peak particle diameter were done in fasting plasma samples by means of nondenaturating gradient gel electrophoresis in the Lawrence Berkeley National Laboratory, University of California,

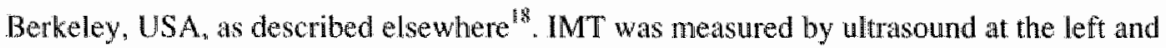
right carotid artery in 17 FCHL subjects as described before ${ }^{17}$. The presence of metabolic 
syndrome in FCHL subjects was assessed according to the World Health Organization (WHO) criteria ${ }^{19}$.

\section{Statistical analysis}

Two-sample $t$ test, adjusted for age and gender was used to analyze differences between FCHL subjects and controls. Log transformed values for triglycerides, insulin, TAT, PAI-1, t-PA and vWF were used in the analyses because these variables showed skewed distribution. Differences in frequencies between groups were tested by chi-square test. The age and gender adjusted Mann-Whitney nonparametric test was used to compare subjects with different lipoprotein phenotypes within the FCHL group, because of small sample sizes $(\mathrm{n}<30)$. Pearson partial correlations controlled for age and gender were used to assess relationship between coagulation factors and metabolic variables in univariate analysis, whereas Spearman rank conrelation was used to describe the relationship between vWF and IMT because of smaller sample size $(n=17)$. In all analyses the statistical package SSPS 11.0 (SSPS Inc., Chicago, Ill.) was used. Statistical significance was defined as $\mathrm{P}<0.05$.

\section{Results}

\section{Coagulation factors}

Anthropometric, metabolic and coagulation system chatacteristics of FCHL subjects and controls are summarized in Table 1 . The hyperlipidlemic FClIL relatives differed significantly from controls in the following age and sex adjusted metabolic variables: higher plasma levels of total cholesterol, triglycerides, LDL-cholesterol, Apo B, insulin, lower HDLcholesterol and predominance of sd LDL particles. FCHL subjects and controls had similat" BMI, but differed significantly in WHR. With regard to the activity profile of the coagutation system and fibrinolytic markers, FCHL subjects showed significantly higher plasma levels of TAT, wWF antigen, F XIIa, PAI- 1 activity and $t$ PA. Plasma levels of D-dimers, STF (with large variations), and sTM did not show significant differences between the groups. There were no group differences in confounding variables (blood pressure, prevalence of CAD, smoking, acetylsalicylate or contraceptive use). 
Table 1. Characteristics of the subjects studied

\begin{tabular}{|c|c|c|c|}
\hline & FCHL Hyperllipidemic Subjects & Controlis & P value \\
\hline No. $(\mathrm{M} / \mathrm{F})$ & $34(15 / 19)$ & $34(18 / 16)$ & \\
\hline Age, yrs & $50.1 \pm 10.9$ & $48.6 \pm 13.0$ & NS \\
\hline TC, mmoll & $7.3 \pm 1.2$ & $5.1 \pm 0.8$ & $p<0.001$ \\
\hline TG*, mmollin & $2.0(1.3-2.5)$ & $1.1(0.8-1.4)$ & $p<0.001$ \\
\hline $\mathrm{HDL}, \mathrm{mmol} / \mathrm{l}$ & $0.9 \pm 0.3$ & $1.0 \pm 0.2$ & 0.015 \\
\hline$L D L$, mmoll & $5.3 \pm 1.2$ & $3.6 \pm 0.7$ & $p<0.001$ \\
\hline LDL size, $\AA$ & $259.6 \pm 8.2$ & $267.1 \pm 5.9$ & $p<0.001$ \\
\hline Apo $\mathrm{B}, \mathrm{g} / \mathrm{I}$ & $1.5 \pm 0.3$ & $1.0 \pm 0.2$ & $p<0.001$ \\
\hline Insulin*, $\mu \mathrm{U} / \mathrm{m} l$ & $7.7(4.3-11.2)$ & $3.4(2-6.4)$ & $p<0.001$ \\
\hline WHA & $0.93 \pm 0.07$ & $0.89 \pm 0.1$ & 0.002 \\
\hline $\mathrm{BM} H, \mathrm{~kg} / \mathrm{m}^{2}$ & $27.3 \pm 4.4$ & $26.0 \pm 3.2$ & NS \\
\hline $\mathrm{SBP}, \mathrm{mm} \mathrm{Hg}$ & $142.9 \pm 15.2$ & $136.8 \pm 23.4$ & NS \\
\hline $\mathrm{DBP}, \mathrm{mm} \mathrm{Hg}$ & $89.0 \pm 9.3$ & $86.5 \pm 12.3$ & NS \\
\hline Prevalence of $C A D^{\dagger}$ & $8(23.5)$ & $3(8.8)$ & NS \\
\hline Statins & $17(50)$ & $2(34)$ & $p<0.001$ \\
\hline Smoking $^{r}$ & $9(26.5)$ & $9(26.5)$ & NS \\
\hline Acetylsalicylate ${ }^{\dagger}$ & $7(20.6)$ & $4(11.8)$ & NS \\
\hline Oral contraceptives ${ }^{\dagger}$ & $1(2.9)$ & $4(11.8)$ & NS \\
\hline \multicolumn{4}{|l|}{ Coagulation Factors: } \\
\hline TAT: $\mu \mathrm{g} / \mathrm{L}$ & $2.6(2.1-2.9)$ & $2.2(2.1-2.3)$ & 0.004 \\
\hline WWF:\% & $109.5(87.8-134.8)$ & $84.0(60.5-123.0)$ & 0.03 \\
\hline FXHa, ng/mL & $1.6 \pm 0.7$ & $1.2 \pm 0.5$ & 0.008 \\
\hline $\mathrm{sTF}, \mathrm{pg} / \mathrm{mL}$ & $53.0 \pm 99.7$ & $53.1 * 118.7$ & NS \\
\hline D-dimers (ng/mL) & $306.3 \pm 164.4$ & $271.7 \pm 180.1$ & NS \\
\hline PAl-1*, U/mL & $16.0(11.8-25.0)$ & $11.0(6.5-15.0)$ & 0.001 \\
\hline $\mathrm{t}-\mathrm{PA} *$ *, ng/mL & $10.7(8.1-13.2)$ & $7.9(4.7-10.9)$ & $p<0.001$ \\
\hline sTM, ng/mL & $31.0 \pm 6.9$ & $30.7 \pm 10.4$ & NS \\
\hline
\end{tabular}

Values are mean $\pm \mathrm{SD}$, *median (interquartile range) and "number ( $\%$ ).

'Two-sample 8 lest, adjusted for age and gender. 


\section{Relation to metabolic syndrome parameters}

We have recently shown that elevation of plasma triglycerides in FCHL is associated with insulin resistance and a number of lipid abnormalities (increased number of VLDL particles, low HDL-cholesterol, preponderance of sd LDL) ${ }^{20}$. Therefore, the present study compared coagulation variables between hyperlipidemic FCHL subjects with elevated plasma ariglycerides ( $\mathrm{TG}>2.3 \mathrm{mmol} / \mathrm{L}$ ) and those with isolated hypercholesterolemia $(\mathrm{TG}<2.3$ mmol/L, Fredrickson type IIa, $n=18$ ). Hypertriglyceridemic FCHL subjects $(n=16)$ represented 9 subjects with combined hyperlipidemia (Fredrickson type ITb), and 7 subjects with isolated hypertriglyceridemia (Fredrickson IV). Hypertriglyceridemic FCHL subjects had significantly higher insulin levels and predoninance of sd LDL particles in comparison to subjects with hypercholesterolemia (Table 2), compatible with presence of features of insulin resistance. This corresponded to significantly higher prevalence of the metabolic syndrome $(62.5 \%)$ among FCHL subjects with hypertriglyceridemia. In addition to these metabolic characteristics, hypertriglyceridemic FCHL subjects had significantly higher PAI-1 activity and STM plasma concentrations. In contrast, no differences were observed in ellevated TAT, F XIIa, TF, D-dimers, t-PA plasma concentrations, and WWF antigen between hypertriglyceridemic and hypercholesterolemic FCHL relatives. Furthermore, one-way ANOVA analysis, with Bonferroni correction as a post hoc test, did not show any statistically significant difference in coagulation markers between hypertriglyceridemic FCHL subjects of type IIb or IV (data not shown). These findings suggested that two different patterns of prothrombotic abnormalities were present in FCHL. One pattern with an increased rate of thrombin generation (TAT), coupled to endothelial activation (vWF, H.PA), was present in all FCHL subjects, independent of phenotype. A different pattern, suggesting impaired fibrinolysis and involving PAI-1 and STM, was predominantly associated to features of the metabolic syndrome, with hypertriglyceridemia as its hallmark in FCHL. To evaluate this possibility further, uniwariate correlations (controlled for age and gender) between metabolic variables and coagulation factors were evaluated in hyperlipidemic FCHL subjects (Table 3). Notably, PAI-1, t-PA, and sTM were linked to variables of the metabolic syndrome by showing a significantly positive relationship to $\mathrm{TG}$ and insulin, and significantly negative correlation with LDL particle size. Furthermore, t-PA correlated positively with WHR. F XIlla was positively correlated with insulin and Apo B. TAT levels and VWF antigen did not show 
Table 2. Comparison between FCHL subjects with a hypercholesterolemic phenotype and FCHE subjects with a hypertriglyceridemic phenotype (IIb, IV)

\begin{tabular}{|c|c|c|c|}
\hline & $\begin{array}{c}\text { Hypercholesterolemic } \\
\text { Phenotype }\end{array}$ & $\begin{array}{l}\text { Hypertriglyceridemic } \\
\text { Phenotype }\end{array}$ & P Value * \\
\hline No. (M/F) & $18(6 / 12)$ & $16(9 / 7)$ & \\
\hline Agle, yrs & $51.3 \pm 11.8$ & $48.7 \pm 10.0$ & NS \\
\hline $\mathrm{TG}^{*}, \mathrm{mmol} / \mathrm{L}$ & $1.4(1.1-1.7)$ & $2.5(2.4-2.9)$ & NA \\
\hline $\mathrm{HDL}, \mathrm{mmol} / \mathrm{L}(\mathrm{M} / \mathrm{F})^{8}$ & $0.8 \pm 0.1 / 1.1 \pm 0.2$ & $0.8 \pm 0.3 / 0.9 \pm 0.2$ & NS \\
\hline LDL, mmol/L & $5.7 \pm 0.8$ & $4.9 \pm 1.4$ & 0.039 \\
\hline LDL size ${ }^{\ddagger}, \AA$ & $265.7 \pm 5.3$ & $254.0 \pm 6.7$ & 0.001 \\
\hline Insiulin ${ }^{*}, \mu \mathrm{U} / \mathrm{ml}$ & $5.4 .(3.6-8.4)$ & $9.3(5.5-14.3)$ & 0.019 \\
\hline WHA & $0.92 \pm 0.07$ & $0.95 \pm 0.05$ & NS \\
\hline$B M 1, \mathrm{~kg} / \mathrm{m}^{2}$ & $26.0 \pm 3.4$ & $27.7 \pm 4.9$ & NS \\
\hline $\mathrm{SBP}_{8} \mathrm{~mm} \mathrm{Hg}$ & $142.1 \pm 16.7$ & $143.7 \pm 13.8$ & NS \\
\hline $\mathrm{DBP}_{\mathrm{m}} \mathrm{mm} \mathrm{Hg}$ & $87.4 \pm 8.8$ & $90.9 \pm 9.8$ & NS \\
\hline Prevalence of $\mathrm{CAD}^{\dagger}$ & $4(22.2)$ & $4(25,0)$ & NS \\
\hline Metabolic syndrome $^{p}$ & $4(22,2)$ & $10(62.5)$ & 0.024 \\
\hline Statins $^{\dagger}$ & $12(66.7)$ & $5(31.6)$ & 0.039 \\
\hline Smoking ${ }^{\dagger}$ & $4(22.2)$ & $5(31.3)$ & NS \\
\hline Acetylsalicylate ${ }^{\dagger}$ & $3(16.7)$ & $4(25.0)$ & NS \\
\hline \multicolumn{4}{|l|}{ Coagulation Factors } \\
\hline TAT $^{*}, \mu g / L$ & $2.5(2.1-3.2)$ & $2.6(2.1-2.8)$ & NS \\
\hline WWF" $\%$ & $107.0(92.0-132.5)$ & $109.5(84.0-146.0)$ & NS \\
\hline $\mathrm{FXlla}, \mathrm{ng} / \mathrm{mL}$ & $1.7=0.9$ & $1.6 \div 0.6$ & NS \\
\hline PAI.1* U/ML & $14.0(9.0-18.0)$ & $24.5(14.3-33.0)$ & 0.002 \\
\hline$\|-P A_{1}{ }^{*} n g / m L$ & $8.5(7.7-13.6)$ & $12.3(10.4-13.2)$ & NS \\
\hline sTM" ${ }^{*} \mathrm{ng} / \mathrm{mL}$ & $28.4 \pm 5.6$ & $33.9 \pm 7.3$ & 0.046 \\
\hline
\end{tabular}

Values are miean is SD. *median (interquhartile range) and tnomber (w).

$\mathrm{NS}=$ not sigmificant. $\mathrm{NA}=\mathrm{nol}$ applicable, because criteria for selection of cases is based on these parameters.

*LDL size below 256 A designates presence of sd LDL; ${ }^{8} \mathrm{HDL}$ cholesterol data ane shown for men and women separate

"Mann Whin 
a significant correlation with any of the tested metabolic traits, but interestingly there was a positive correlation between wWF antigen levels and IMT (rho $=55, \mathrm{p}<0.05$ ), (Figure 1). When coagulation factors were correlated with each other, there was a significant positive correlation between $v$ WF and t-PA $(r=.54, p<0.01)$, and between vWF and TAT $(r=47$, ). The expected correlation between PAI-1 and $\mathrm{BA}$ was present, although it did not reach statistical significance $(r=.39, p=0.07)$.

Table 3. Pearson Correlation Coefficients between coagulation factors in plasma and metabolic and anthropometric variables in hyperlipidemic FCHL subjects.

\begin{tabular}{lcccccccc}
\hline $\begin{array}{l}\text { Coagulation } \\
\text { Factors }\end{array}$ & TC & LDL & ApoB & TG & LDL size & Insullin & BMI & WHR \\
\hline TAT & - & - & - & - & - & - & - & - \\
VWF & - & - & - & - & - & - & - & - \\
FXIla & - & - & $.41^{*}$ & - & - & $.42^{*}$ & $.33^{\dagger}$ & - \\
PAIl-1 & - & - & - & $.62^{\star * *}$ & $-.58^{* *}$ & $.46^{*}$ & $.34^{*}$ & - \\
t-PA & - & - & - & $.61^{* *}$ & $-.46^{*}$ & $.58^{* *}$ & - & $.43^{*}$ \\
sTM & - & - & - & $.46^{*}$ & $-.36^{\S}$ & $.35^{*}$ & $.32^{\S}$ & - \\
\hline
\end{tabular}

* $\mathrm{p}<0.05, * \mathrm{p}<0.01, * * \mathrm{p}<0.001$, age and gender adjusted

$\dagger \mathrm{P}=0.09,+\mathrm{P}=0.07,8 \mathrm{P}=0.06{ }^{\circ 6}{ }^{4+}=$ Not statistically signilicant

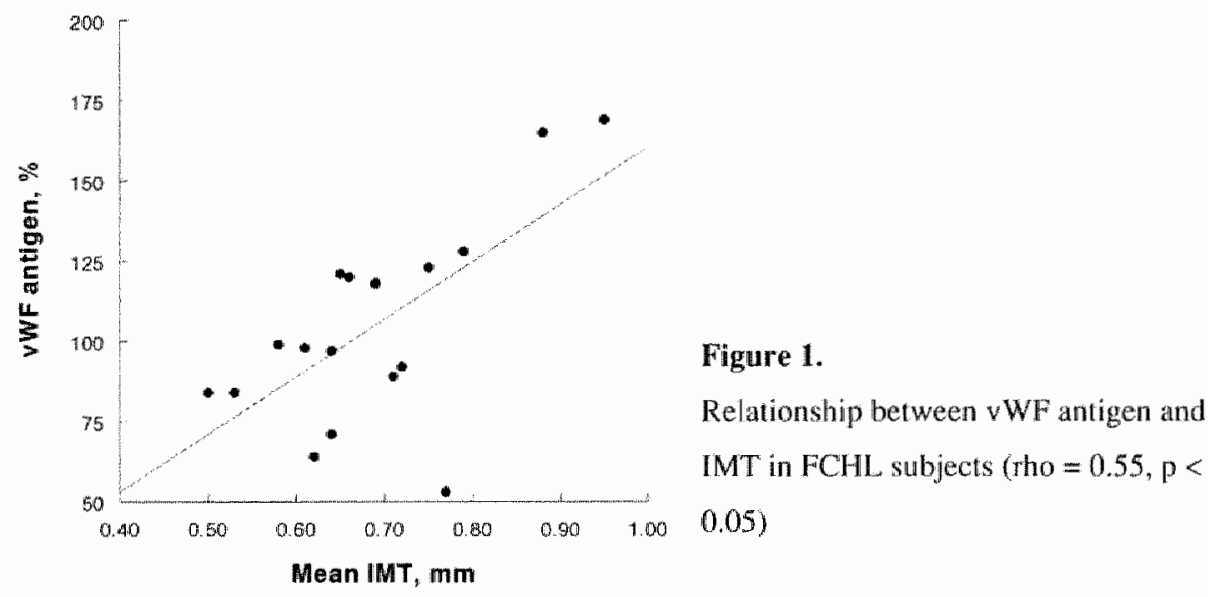




\section{Discussion}

The present study is the first to evaluate several components of the blood coagulation system in hyperlipidemic FCHL subjects, reported to be al increased risk of premature myocardial infarction ${ }^{1-3}$. Hyperlipidemic FCHL subjects exhibited a prothrombotic phenotype, indicated by significantly higher plasma concentrations of TAT, F XIIa, vWF, and t-PA, in comparison to controls. These results are interpreted as increased coagulation activity coupled to activation of vascular endothelium. Within the subgroup of FCHL subjects with a hypertriglyceridemic phenotype, elevated PAI-I activity and sTM levels were particularly associated with features of the metabolic syndrome (hyperinsullinemia, hypertriglyceridemia, predominance of sd LDL). Therefore, two different patterns of procoagulant abnormalities in FCHL emerged from the present findings. The limited number of cases with FCHL, however, warrants some caution in interpretation of the results.

The observed increased prothrombotic activity in FCHL subjects can be explained by endothelial activation, or more advanced atherosclerotic lesions. We showed previously that the carotid artery intima-media thickness, a validated surrogate marker of atherosclerosis, is increased in FCHL compared to control subjects ${ }^{17}$. Therefore, it is plausible that the increased prothrombotic activity in hyperlipidemic FCHL subjects reflects a process of active atherothrombosis. According to current insights, the process of atherosclerotic plaque progression is often accompanied by episodes of thrombosis triggered by nupture or erosion, but many of these thrombi remain mural rather than occlusive ${ }^{6,21}$. Such a mechanism can underlie the increased basal coagulation activity in plasma of hyperlipidemic (both with elevated TG or cholesterol) FCHL, subjects, as suggested by significantly elevated TAT plasma levels. TAT complex formation results from thrombin generation and subsequent binding to its inhibitor antithrombin. Enhanced generation of thrombin may have different origins, some of which may be increased exposure of tissue factor on circulating cells or microparticle fragments, or by plaques ${ }^{21}$. However, it is not known at present whether such a mechanism of TAT activation occurs in stable or vulnerable plaques ${ }^{17}$, or both. Alternatively, increased TAT plasna concentrations may be explained by increased platelet activation. This altemative explanation is supported by the significantly positive correlation between TAT concentrations and WWF antigen, which is known to play a major role in platelet adhesion, 
and perhaps, in the initiation of atherosclerosis. VWF behaves like an acute phase protein that can be released from endothelial cells in response to different types of cell injury. Endothelial dysfunction with reduced NO generating activity has been previously documented in $\mathrm{FCHL}^{13}$. Furthermore, vWF antigen showed positive relationship with IMT in this study. Therefore, the present data suggest that activated endothelium ${ }^{13.22}$, plaque development and possibly platelet activation, underlie increased TAT and VWF plasma levels in FCHL.

The specific significance of F XIIa in FCHL is still unknown. Recent cardiovascular studies suggest it to be a marker of increased risk for coronary complications but the pathophysiological significance of this contact protein for fibrin formation is disputed ${ }^{8}$. The origin of increased factor XIIa activation is generally unknown but the present data suggest that an increased number of lipoprotein particles, measured as elevated plasma Apo B in FCHL, may be involved, probably by providing a surface mediated activation of the zymogen F XII. An unexpected association between F XIla and the metabolic factors (BMI, insulin levels; Table 3) may suggest an additional role of adipocytes or other cells in this regard.

Hyperlipidemic FCHL subjects showed elevated PAI-1 activity in the present study. Specifically, hypertriglyceridemic FCHL subjects showed a highly significant 2-fold elevated PAI-1 activity, in combination with mild elevation of sTM levels, compared to hypercholesterolemic FCHL subjects. This finding is of interest because recent studies have shown that cardiovascular risk in FCHL is strongly related to the presence of metabolic dyslipidemia $^{4}$, and the excess of atherosclerosis and impaired fibrinolysis respectively ${ }^{28}$. Whereas the association of STM with the metabolic syndrome has not been studied, increased PAI-1 activity is linked to underlying metabolic abnormalities such as hyperinsulinemia, hypertriglyceridemia, and increased plasma $\mathrm{FFA}^{23.24}$. In type $2 \mathrm{DM}$, another insulin resistant state, adipocytes have been shown to be important factor in the production of PAI- ${ }^{25}$. However, in FCHL an additional mechanism may be operational because a major difference in PAI-1 activity existed between the hypercholesterolemic and hypertriglyceridemic FCHL. subjects, but BMI and WHR were not significantly different (Table 2). Recent studies provided evidence of important contribution of the liver, specifically fatty liver, to plasma PAI-1 levels $\mathrm{s}^{26,27}$, and the high prevalence of nonalloholic fatty liver disease in hypertriglyceridemic FCHL subjects ${ }^{28}$ is a potential explanation of elevated PAI-1 activity. Furthermore, FCHL is linked and associated with the gene encoding upstream transcription 
factor 1 (USFI), especially in subjects with high triglycerides ${ }^{29}$. Interestingly, PAI-1 gene activity can be regulated by $\mathrm{USF}^{2} \mathrm{I}^{30}$, and therefore $\mathrm{PAI}-1$ is a potential marker, that reflects USF-1 activity in FCHL. Thus, elevated activity of PAI-1 in hypertriglyceridemic FCHL subjects can be particularly inherent to the nature of FCHL.

In summary, the present findings are suggestive of two types of prothrombotic abnormalities in hyperlipidemic FCHL subjects. A general pattern of activated blood coagulation and endothelial activation, which may relate to plaque burden, is present in all. hyperlipidemic subjects, independent of metabolic phenotype. In those FCHL subjects with features of the metabolic syndrome, specifically the hypertriglyceridemic phenotype, inhibited fibrinolytic mechanisms may provide an additional cardiovascular risk factor. The present findings advance our understanding of the complex relationship between lipoprotein, metabolic phenotypes, and haemostatic characteristics of plasma in FCHL, and may have implications for therapy and prevention of acute coronary events in hyperlipidemic and insulin resistant disorders.

\section{Acknowledgements}

We thank Mrs. Josepline wan Lin, research nurse, for the recruitment of FCHL patienis; Martijn C.G.J.

Brouwers for discussions, and Steven $\mathbf{J}$. Meex for statistical adwice. Anna M. Georgieva has been supported by a Marie Curie Fellowship of the Europeam Community program 'Quality of Life and Management of Living Resoures" under contract mumiber QLKS-CT-2000-60007, and a Job Cohen Grant of the Maastricht University. Hugo ten Catte is a Clinical Established Investigator fron the Netherlands Heart Foundation. Tjerk W.A. de Bruin has been supported by a gran of the Netherlands Organization for Scientific Research (no. 900-95-297), This research was atso supported by CARIM, Candiovascular Research Institute Maastricht and the Academic Hospitin Menstuctit. 


\section{References}

1. Goldstein JL, Schrott HG, Hazzard WR, et al. Hyperlipidenia in coronary heart disease. II. Genetic analysis of lipid levels in 176 families and delineation of a new intherited disorder, combined hyperlipidemia. J Clin Imvest. Jul 1973;52(7):1544-1568.

2. Voors-Pette C, de Bruin TW. Excess coronary heart disease in Familial Combined Hyperlipidemia, in relation to genetic factors and central obesity. Atherosclerosis. Aug 2001:157(2):481-489.

3. Hopkins PN, Heiss G, Ellison RC, et al Coronary Artery Disease Risk in Familial Combined Hyperlipidemia and Familial Hypertriglyceridemia. A Case-Control Comparison From the National Heart, Lung, and Blood Institute Family Heart Study. Circalation. Jul 72003.

4. vary der Kallen CJ, Voors-Pette C. Bouwman FG, et al. Evidence of insulin resistant lipid metabolism in adipose tissue in fanilial combined thyperlipidemin, but not type 2 diabetes mellitus. Atherosclerosis. Oct 2002;164(2):337-346.

5. Maseri A, Fuster V. Is there a vulnerable plaque? Circulation. Apr 29 2003; 107(16):2068-2071.

6. Kereiakes DJ. The Emperor's clothes: in search of the vulnerable plaque. Circulation. Apr 29 2003:107(16):2076-2077.

7. Ridker PM. Evaluating nowel cardiovascular risk factors: can we better predict heart attacks? Am Intern Med. Jun I1999; 130(11);933-937.

8. Zito $F$, Drummond $F$, Bujac SR, et al. Epidemiological and genetic associations of activated factor XII concentration with factor VII activity, fibrinopeptide A concentration, and risk of coronary hetru disease in men. Circulation. Oct 24 2000;102(17):2058-2062.

9. Meigs JB, Mittleman MA, Nathan DM, et al. Hyperinsulinemia, hyperglycernia, and impaired thenostasis: the Framingham Offspring Study. Jama. Jan 12 2000;283(2):221-228.

10. Thogersen AM. Jansson JH. Boman K, et al. High plasminogen actiwatov inhibitor and tissue plasminogen activator levels in plasma precede a first acute myocardial infarction in botll then and women: evidence for the fibrinolytic system as an independent primary risk factor. Circulation. Nov 24 1998;98(21):2241-2247.

11. Anand SS, Yi Q, Gerstein H, et al. Relationship of metabolic syndrome and fibrinolytic dysfunction to cardiovascular disease. Circulation. Jul 29 2003;108(4):420-425.

12. Sramek A, Kriek M, Rosendaal FR. Decreased mortality of ischaemic heart disease annong carriers of thaemophilia Lancer. Aug 2 2003:362(9381):351-354.

13. Stroes $\mathrm{E}$, de Bruin $\mathrm{T}$, de Valk $\mathrm{H}$, et al. NO activity in familial combined thyperlipidemilat potential role of cholesterol remnants. Cardiovasc Res. Dec 1997:36(3):445-452.

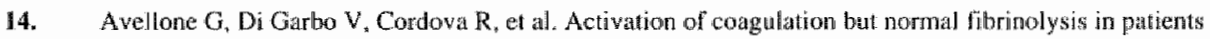
with type IIA hyperlipoproteinemia. Thromb Res. Jan 15 1996;81(2):277-282.

15. Hiraga T, Shimada M, Tsukada T, et al. Hypertriglyceridemia, but not hypercholesterolemia, is associated with the alterations of fibrinolytic system. Morm Metab Res. Nov 1996;28(11):603-606. 
16. Lundman P, Eriksson MJ, Silweira A, et all, Relation of hypertriglyoeridemia to plasma concentrations of biochemical markers of inflammation and endotheliat activation ( $C$-reactive protein, interleukin- 6 , soluble adhesion molecules, von Willebrand factor, and endothelin-1). Am I Cardiol. May 1 2003:91(9):1128-1131.

17. Ketlen ET, Kruigsshoop M, Schaper NC, et al. Increased intima-media thickness in familial combined hyperlipidemia associated with apolipoprotein B. Arterioseler Thromb Vasc Biol. Feb 1 $2002 ; 22(2): 283-288$.

18. Allayee $H$, Domingue $\mathrm{KM}$, Aouizerat $\mathrm{BE}$, et al. Contribution of the hepatic lipase gene to the atherogenic lipoprotein phencotype in familial combined hyperlipidemia. J Lipid Res. Feb $2000 ; 41(2): 245-252$

19. Lakka HM, Laaksonen DE, Lakka TA, et al. The metabolic syndrome and total and cardiovascular disease mortality in middle-aged men. Jama. Dec 4 2002;288(21):2709-2716.

20. Georgieva AM, Van Greevenbroek MM, Krauss RM, et al. Subclasses of Low-Density Lipoprotein and Very Low-Density Lipoprotein in Familial Combined Hyperlipidemia: Relationship to Multiple Lipoprotein Phenotype. Arterioscler Thromb Vasc Biol. Jan 292004.

21. Fuster V, Fayad ZA, Badimon JJ. Acute coronary syndromes: biology. Lancet. Jun 1999;353 Suppl 2.suis-9.

22. Bonetti PO, Lerman LO, Lerman A. Endothelial dysfunction: a marker of atherosclerotic risk. Arteriosicter Thromb Vasc Biol. Feb 1 2003;23(2):168-175.

23. Kohler HP, Grant PJ. Plasminogen-activator inhibitor type 1 and coronary artery disease. N EnglJ Med. Jun $1.52000 ; 342(24): 1792-1801$.

24. Reaven G. Metabolic syndrome: pathophysiology and implications for management of cardiovascular disease Circulation. Jul 16 2002;106(3):286-288.

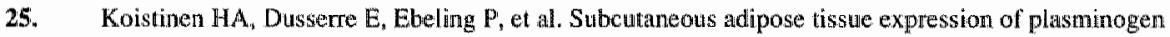
activator inhibitor 1 (PAI-1) in nondiabetic and Type 2 diabetic subjects. Diabetes Metab Res Rev. SepOet 2000; $16(5): 364-369$.

26. Yki-Jarvinen H, Sutinen J, Silweira A, et al. Regulation of Plasma PAI-1 Concentrations in HAARTAssociated Lipodystrophy During Rosiglitazone Therapy. Arterioscles Thromb Vase Biol. Apr 1 $2003: 23(4): 688.694$

27. Alessi MC, Bastelica D, Mavri A, al Plasma PAI-1 Levels Are More Strongly Related to Liver Steatosis Than to Adipose Tissue Accunulation. Arterioscler Thromb Vasc Biol. Jul 1 $2003 ; 23(7) \div 1262-1268$.

28. de Bruin T GA, Brouwers M, Heitink M, van cler Kallen C, Greevenbroek M. Radiological evidence of nonalcoholic faty liver disease in famihial combined hyperlipidemia. The Annerican Joumal of Medicine in press.

29. Pajukanta P, Lilja HE. Sinsheimer JS, et al. Familial combined hyperlipidenia is associated with upstream iranscription factor \(USFI). Nar Genet. Feb 292004. 
30. Zietz B. Drobnik W, Herfarth H, et all. Plasminogen activator unhibitor-1 promoter activity in adipocytes is not influenced by the $4 \mathrm{G} / 5 \mathrm{G}$ promoter polymorphism and is negulated by a USF-1/2 binding site immediately preceding the polymorphic region. J Mol Endacrinol. Feb 2004:32(1):155-163. 
Chapter 5

\section{Very Low-Density Lipoprotein Subclass Distribution in}

\section{Familial Combined Hyperlipidemia; Relationship to}

\section{Hypertriglyceridemia and Metabolic Syndrome.}

Anna M. Georgieva, Marleen M.J. wan Greevenbroek, Martijn C.G.J. Brouwers, Carla J.H. wan der Kallen, Tjerk W.A. de Bruin

Submitted for publication 


\section{Abstract}

The objective of the present study was to get more insight on the lipoprotein abnormalities underlying hypertriglyceridemia in FCHL. We examined the relationship between VLDL subclasses and plasma triglycerides in FCHL and controls, and evaluated a potential association between the VLDL heterogeneity and the presence of metabolic syndrome in FCHL. VLDL1 and VLDL2 fractions were isolated from 44 hyperlipidemic FCHL subjects and 18 normolipidemic controls by means of density gradient ultracentrifugation and biochemically characterized. In FCHL subjects a redistribution of plasma triglyoerides (TG) towards lipoprotein fractions of lower density, with significantly higher VLDL1-Apo B levels was observed compared to controls $(p<0.01)$. Assessment of TG to Apo B ratios indicated that VLDLI lipoproteins were of normal size in FCHL. VLDL1-TG explained more than $80 \%$ of the variability of total plasma TG in FCHL study population. FCHL subjects with elevated plasma VLDL1-TG ( $>0.5 \mathrm{mmol} / \mathrm{L}$ ) were more likely to have an extended form of metabolic syndrome, defined as the presence of 3 or more ATPII criteria in addition to elevated plasma TG $(p<0.01)$. In conclusion, hypertriglyceridemia in FCHL is associated with overproduction and/or delayed elimination of VLDL1 lipoproteins of normal size. Numerical expansion of plasma VLDL1 lipoproteins is a biochemical characteristic of FCHL subjects with full-blown metabollic syndrome. 


\section{Introduction}

Familial Combined Hyperlipidemia (FCHL) was originally identified as a genetic disorder of lipid metabolism, associated with an increased risk of premature coronary artery disease $(C A D)^{\Downarrow}$. However, the pathogenesis of FCHL can extend beyond lipid metabolism. Affected FCHL subjects often exhibit a complex insulin resistant phenotype with a high prevalence of metabolic syndrome components, such as abdominal obesity, hypertriglyceridemia and hypertension ${ }^{2,3}$. A recent study identified the presence of metabolic syndrome, as defined by National Cholesterol Education Program Adult treatment panel III (ATP III), in the majority of FCHL subjects $(65 \%)$ compared to $19 \%$ in controls ${ }^{4}$.

Hypertriglyceridemia in FCHL is known to be associated with the presence of small dense LDL, and often low HDL cholesterol ${ }^{5}$. This phenotype has been previously designated as the Atherogenic Lipoprotein Phenotype (ALP), because of its association with an increased risk for $\mathrm{CAD}^{6}$. Such a cluster of lipid abnormalities, which probably results from altered metabolism of VLDL1 particles against a background of insulin resistance ${ }^{5}$, is often present in the metabolic syndrome as well ${ }^{7}$. A specific genetic susceptibility for the ALP has been reported in FCHL 6 .

Hypersecretion of hepatic Apo B-containing lipoproteins, VLDL-Apo B in particular, can represent a common pathological background of hypertriglyceridemia in FCHL and the metabolic syndrome ${ }^{8,9}$. Apo-B containing lipoproteins are synthesized in the hepatocyte, where neutral lipid is combined with Apo B protein in a multi-step process, regulated, at least in part, by insulin ${ }^{10,4}$. The amount of lipid added during lipoprotein assembly determines the size (density range) of VLDL lipoproteins that are secreted by the liver, i.e. VLDL1 (Sf 60 to 400 ) or VLDL2 (Sf $20-60)^{12}$. Enhanced production rate of VLDL1, but not VLDL2 have been associated with insulin resistance and accounts for the dyslipidemia in type 2 DM and the metabolic syndrome ${ }^{13}, 14$. In order to get more insight on the lipoprotein abnormalities underlying hypertriglyceridemia in FCHL, we characterized VLDLI and VLDL2 subclasses in FCHL subjects and controls with regard to lipid and apolipoprotein content. The rellationship between VLDL subclasses and plasma triglycerides was examined. In addition, association between the presence of the metabolic syndrome and VLDL heterogeneity in FCHL was evaluated. 


\section{Subjects and Methods}

FCHL probands and controls were recruited through the Lipid Clinic of the Maastricht University Hospital in the framework of genetic and metabolic studies. A total of 44 hyperlipidemic FCHL subjects and 18 normolipidemic controls were included. The subjects represented FCHL families ( $n=19$ ) ascertained as previously described ${ }^{3}$. Briefly, FCHL. probands had: 1) primary hyperlipidemia with varying phenotypic expression, including (untreated) fasting plasma cholesterol $>6.5 \mathrm{mmol} / 1$ and/or fasting plasma triglyceride concentration > $2.3 \mathrm{mmol} / \mathrm{l} ; 2$ ) they were known with premature $\mathrm{CAD}$ (before the age of 60 ), or had a positive family history of such; 3) had at least one first-degree relative with a different lipoprotein phenotype. Exclusion criteria for diagnosing FCHL in a family were diabetes mellitus type 2, obesity (BMI $>30$ ), causes of secondary hyperlipidemia (nephrotic syndrome, hypothyroidism, morbid obesity), E2/E2 genotype or tendon xanthomas of the proband.

Presence or absence of metabolic syndrome was identified in all subjects according to ATP III definition as three or more of the following criteria ${ }^{7}$ : 1) abdominal obesity (waist circumference: $>102 \mathrm{~cm}$ in men, and $>88 \mathrm{~cm}$ in women); 2) hypertriglyceridemia ( $\geq 1.7$ $\mathrm{mmol} / \mathrm{l})$ : 3 ) low HDL cholesterol ( $<1.00 \mathrm{mmol} / \mathrm{l}$ in men, and $<1.29 \mathrm{mmol} / \mathrm{l}$ in women); 4) hypertension ( $\geq 130 / \geq 85 \mathrm{mmHg}$ ) or medication; 5) impaired whole blood fasting glucose $(\geq$ $5.6 \mathrm{mmol} / 1)$

Subjects were studied after an overnight fast (12-14 h) and 3 days without alcohol consunption. Any lipid lowering medication was stopped for two weeks before blood samples were collected. The Human Investigation Review Committee of the Academic Hospital Maastricht approved the study protocol. All subjects gave informed consent.

Venous blood was collected in pre-cooled EDTA ( $1 \mathrm{mg} / \mathrm{mL})$ containing tubes. Anthropometric measurements (calculation of BMI), and measurements of fasting plasma concentrations of lipids, lipoproteins, and insulin were done as described ${ }^{3}$. Total plasma VLDL $\left(<1.006 \mathrm{~g} / \mathrm{ml}\right.$ ) was isolated according to the method of Redgrave et al. ${ }^{15}$ and subsequently fractionated into VLDL1 and VLDL2 by density gradient ultracentrifugation at $160000 \mathrm{~g}$ for 2.5 hours at $4^{\circ} \mathrm{C}$ in SW40 Ti rotor as described ${ }^{16}$. Collection of fractions started from the top of the tube, where the upper $1.5 \mathrm{ml}$ represented VLDL1 and the lower $5 \mathrm{ml}$ 
VLDL2. Measurements of triglycerides and cholesterol in the subfractions were done by standard laboratory techniques. Concentration of Apo B in VLDL1 and VLDL2 fractions was measured by gel electrophoresis according to the method of Karpe et al ${ }^{17}$. Apo B concentration in a density fraction reflects the number of lipoproteins, because there is only one Apo B molecule per lipoprotein particle.

\section{Statistical analyses}

Log transformation was used for values of triglycerides (TG), insulin, VLDL $1-$ TG, VLDL2-TG, VLDL1-TG/VLDL2-TG ratio, and TG/Cholesterol ratio, because of skewed distribution of these variables. General linear model was used to examine differences between controls and FCHL subjects, and differences in VLDL subclasses, after correction for age, gender and BMI. This correction was done because gender, age and BMI have been shown to affect VLDL metabolism and plasma triglycerides ${ }^{16,18}$. Mann-Whitney nonparametric test was used for the Apo B (Table 2) and the subgroup analyses (Table 4) because of small sample sizes. Statistical significance was defined at $\mathrm{P}<0.05$. Multiple regression analysis was used to define predictors of plasma TG, and VLDL1/VLDL2-TG ratio "BMI, age, gender, insulin and FCHL status (yes/no) were entered as independent wariables in all models. Variables were eliminated by stepwise backwards regression analysis until all remaining variables had a significance level of $P<0.05$. In all analyses, the statistical package SSPS 11.0 (SSPS Inc., Chicago, IL) was used.

\section{Results}

Characteristics of the study groups are presented in Table 1. By definition, hyperlipidemic FCHL subjects $(n=44)$ showed significant differences with normolipidemic controls $(n=18)$ in plasma lipid profile (corrected for age, gender, and BMI). Of the hyperlipidemic FCHL subjects, $68.2 \%$ had 3 or more metabolic syndrome features, and therefore fulfilled the ATP III definition of the metabolic syndrome, compared to $27.8 \%$ of the normolipidemic controls $\left(\chi^{2}, p<0.01\right)$. 
Table 1. Characteristics of study groups

\begin{tabular}{lccc}
\hline & Controls & FCHL & P value \\
\hline No. (M/F) & $18(9 / 9)$ & $44(20 / 24)$ & \\
Age, $y r s$ & $53.5 \pm 14.7$ & $52.4 \pm 12.2$ & NS \\
BMl, $\mathrm{kg} / \mathrm{m}^{2}$ & $26.2 \pm 3.8$ & $27.7 \pm 4.5$ & NS \\
Waist, cm & $92.9 \pm 12.0$ & $96.7 \pm 11.3$ & NS \\
TC, mmol/L & $5.1 \pm 0.4$ & $6.8 \pm 1.2$ & .0001 \\
Apo B, g/L & $1.0 \pm 0.2$ & $1.4 \pm 0.3$ & .0001 \\
TG, mmol/L & $1.0 \pm 0.3$ & $2.1 \pm 0.9$ & .0001 \\
HDL-chol, mmol/L & $1.1 \pm 0.2$ & $0.9 \pm 0.3$ & .02 \\
Glucose, $\mathrm{mmol} / \mathrm{L}$ & $4.9 \pm 0.7$ & $5.1 \pm 0.8$ & NS \\
Insulin, $\mu \mathrm{U} / \mathrm{ml}$ & $3.4(2.0-5.4)$ & $7.1(5.1-10.4)$ & .001 \\
SBP, mmHg & $132 \pm 15$ & $141 \pm 20$ & NS \\
DBP, mmHg & $84 \pm 7$ & $88 \pm 11$ & NS \\
Prevalence of MetS & $5(27.8)$ & $30(68.2)$ & .002 \\
\hline
\end{tabular}

* $P$ values after correction for age, gender, and BMI

Values are mean $\pm \mathrm{SD}$, median (interquartile range), and number ( $(\%)$

Abbreviations: $\mathrm{M} / \mathrm{F}=$ male/ female, $\mathrm{BMI}=$ body mass index, $\mathrm{TC}=$ total cholesterol, $\mathrm{Apo} \mathrm{B}=$ apolipoprotein $\mathrm{B}$, TG= triglycerides. HDL-chol= high density lipoprotein cholesterol, $\mathrm{SBP}=$ systolic blood pressure, $\mathrm{DBP}=$ diastolic blood pressure, MetS = metabolic syndrome, NS = not significant

\section{Characteristics of VLDL1 and VLDL2 in hyperlipidemic FCHL subjects and normolipidemic controls.}

FCFHL, subjects had significantly higher amount of triglycerides in VLDL1 (3-fold increase. $p<0.001$ ) and VLDL2 fractions $(1.5$-fold, $p<0.02)$ compared to normolipidemic controls (Table 2), as well as more cholesterol in both VLDL 1 ( 5 -fold increase, $p<0.001$ ) and VLDL2 (2-fold, p<0.02). Moreover, FCHL subjects had a higher number of VLDL1 and VLDL2 lipoproteins than controls, measured as concentration of Apo B, with a major, 5-fold increase in VLDL1-Apo $B(p<0.01)$, and a modest increase in VLDL2 $(1.5$-fold, $p<0.05)$. 
Altogether, the increase in lipid and Apo B was more distinct in VLDL1 in FCHL subjects. This resulted in the significantly 2-fold increased ratio of VLDL1/VLDL2 TG in FCHL compared to controls. These observations combined indicated a shift in distribution of plasma triglyceride towards VLDL lipoproteins of lower density (VLDL1).

Triglyceride content per particle (TG/Apo B ratio) in VLDL subfractions did not differ between FCHL subjects and controls (Table 2). Of note, the size of the VLDLI particle is largely determined by its triglyceride content, because triglycerides account for more than 65 \% of the total lipid content of VLDLI lipoproteins ${ }^{19}$. Thus, our analysis suggested that VLDL1 lipoproteins in FCHL have similar size $(25 \pm 9 \mu \mathrm{mol} / \mathrm{mg})$ as in controls $(33 \pm 8$ $\mu \mathrm{mol} / \mathrm{mg}$, NS). The cholesterol content per particle (Chol/Apo B ratio) however was increased in VLDL1 of FCHL subjects $(9 \pm 4 \mu \mathrm{mol} / \mathrm{mg})$ compared to controls $(6 \pm 1$ $\mu \mathrm{mol} / \mathrm{mg}$, NS), and VLDL2 (9 $\pm 3 \mu \mathrm{mol} / \mathrm{mg} \mathrm{vs} .4 \pm 2 \mu \mathrm{mol} / \mathrm{mg}, \mathrm{p}<0.01)$. Moreover, the TG/Chol molar ratio for both VLDL subfractions was significantly lower in FCHL subjects than in controls. This additional finding supported the observation that VLDL lipoproteins in FCHL were relatively enriched in cholesterol. 
Table 2. Characteristics of VLDLI and VLDL2 in hyperlipidemic FCHL subjects and normolipidenic controls

\begin{tabular}{|c|c|c|c|}
\hline & Controls & $\mathrm{FCH} \mathbb{L}$ & P value \\
\hline N & 18 & 44 & \\
\hline \multicolumn{4}{|l|}{ VLDL 1} \\
\hline$T G_{i}$ mmoll & $0.22 \pm 0.17$ & $0.66 \pm 0.58$ & .0001 \\
\hline Chol, minoliL & $0.06=0.05$ & $0.31 \pm 0.39$ & .0001 \\
\hline Apo $B_{w} \mu \mathrm{g} /\left.\mathrm{m}\right|^{\| \prime}$ & $9.4 \pm 8.4$ & $42.8 \pm 25.7$ & .002 \\
\hline TGIApo B, $\mu \mathrm{m}$ ol/mg" & $33 \pm 8$ & $25 \div 9$ & NS \\
\hline Chall Apo B, molimg $^{\prime \prime}$ & $6 \pm 1$ & $9 \pm 4$ & NS \\
\hline TG/ Chol & $3.9(2.6-5.4)$ & $2.6(1.9-3.1)$ & .03 \\
\hline
\end{tabular}

VLDL 2

TG, mmol/L

$0.21 \pm 0.09$

$0.32 \pm 0.17$

.015

Chol, mmol/L

$0.11 \pm 0.06$

$0.22 * 0.14$

.006

Apo B, $\mu \mathrm{g} /\left.\mathrm{m}\right|^{* t}$

$24.1 \pm 9.6$

$37.2 \pm 7.8$

.026

TG/Apo B, $\mu \mathrm{mol} / \mathrm{mg}^{\mathrm{t}}$

$12 \pm 3$

$12 \pm 3$

.NS

Chol/ Apo B B $_{\text {mol/mg" }}$

$4 \pm 2$

$9 \pm 3$

.005

TG/Chol

$1.9(1.5-3.0)$

$1.5(1.1-2.0)$

.04

VLDL1NLDL2 -TG

$0.7(0.6-1.3)$

$1.4(1.0-2.4)$

.001

pralue, after correction for BMll age and gender

"For the Apolpoprotein B analysis: controls $\mathrm{n}=6$; $\mathrm{FCHL} \mathbf{n}=9$; differences were ewaluated by Mann-Whitney nonparametric test. 


\section{Dissection of determinants of plasma TG concentrations}

We used several models to evaluate which variables predicted plasma TG (Table 3 , Model $A_{3}$ ). VLDL1-TG was the most important predictor of the variation in plasma TG (Table 3. Figure 1), whereas FCHL status contributed only weakly to the model $\left(\mathrm{R}^{2}\right.$ change $=.030$, $\mathrm{p}<0.01$ ). By contrast, the other predictors in the model: VLDL2-TG, plasma insulin, BMI, age and gender did not contribute significantly to variation in plasma TG. This modell suggested that VLDL1-TG explained most of the variation that alternatively would have been carried by "FCHL affected status".

Similarly, when FCHL subjects were analyzed separately, VLDL1-TG predicted $84 \%$ of the variability in plasma $\mathrm{TG}\left(\mathrm{R}^{2}=.835, \mathrm{p}<0.001\right)$. Subsequent analyses showed a highly significant positive relationship between VLDL1-TG concentrations and the amount of VLDL 1-Apo B (rho $=.946, p<0.00 \mathrm{~L}$ ). Combined with the data discussed in the previons paragraph, this analysis confirmed that the increase in plasma triglyceride concentrations in FCHL subjects was associated with an increased number of VLDL1 lipoproteins in plasma, rather than with an increased TG content per VLDL1 particle.

Table 3. Dissection of determinants of plasma TG concentration. Predictors of plasma TG, and VLDL1/VLDL2 TG ratio were evaluated by multiple backwards regression analysis.

\begin{tabular}{|c|c|c|c|c|c|c|}
\hline Varlable & Predictors & $\bar{N}$ & Stand. $\beta$ & $\mathbf{P}$ & $\mathrm{R}^{2}$ & $R^{2}$ Change \\
\hline \multirow[t]{2}{*}{ A: Plasma $T \bar{G}^{T}$} & VLDL1-TG & 62 & .809 & .000 & 820 & \\
\hline & $\mathrm{FCHL}$ & & 203 & .001 & .850 & .030 \\
\hline \multirow[t]{2}{*}{ B: VLDL1/NLDL2-TG ${ }^{2}$} & Plasma TG & 62 & .620 & .000 & .556 & \\
\hline & Insullin & & .244 & .014 & .599 & .044 \\
\hline
\end{tabular}

\footnotetext{
Model A included VLDL1-TG, VLDL2-TG, insulin, BMI, age, gender wind FCHL status.

2 Model B included plasma TG; insulin, BMI, age, gender and FCHL status,
} 


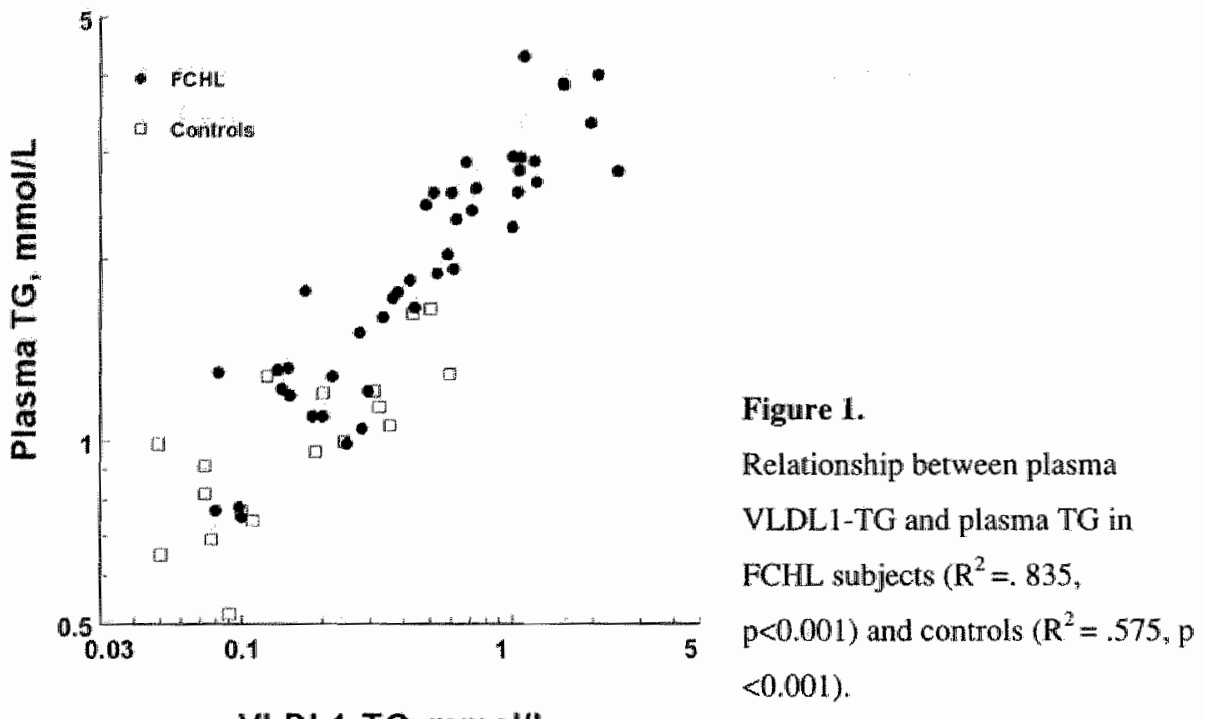

Subsequently, we evaluated the factors that determined the distribution of plasma TO between the VLDL subclasses, i.e. VLDL1/VLDL2-TG ratio (Table 3. Model B). Interestingly, plasma insulin predicted significantly the VLDLL/VLDL2-TG ratio, even after correction for plasma triglycerides. This indicated that the shift in triglyceride distribution towards lipoproteins of lower density occurred in the face of elevated plasma insulin levels.

\section{Presence of metabolic syndrome: relationship to VLDL subclasses}

We examined the effect of the presence of the metabolic syndrome on the VLDL heterogeneity by subgroup analysis. For the purpose of this analysis only healthy controls, i.e. without metabolic syndrome were included.

Characteristics of VLDL1 and VLDL2 in FCHL subjects with and without metabolic syndrome, and of healthy controls are presented in Table 4. Comparison was made between FCFL subjects with and without metabolic syndrome, as well as between FCHL subjects without metabolic syndrome and healthy controls. FCHL subjects with metabolic syndrome had significantly more triglycerides and cholesterol, particularly in VLDLI (TG: $0.87 \pm 0.61$ mmol/l; Chol: $0.41 \pm 0.43 \mathrm{mmol} / \mathrm{l}$ ) than FCHL subjects without the metabolic syndrome (TG: $0.24 \pm 0.13 \mathrm{mmol} / \mathrm{L}$, Chol: $0.1 \pm 0.07 \mathrm{mmol} / \mathrm{h}, \mathrm{p}<0.001$ for both). Moreover, the 
VLDL1/VLDL2 TG ratio was higher in FCHL subjects with metabolic syndrome [1.6(1.2 $3.9)]$ than those without metabolic syndrome [1.1 $(0.8-1.4), p=0.02]$. These obserwations indicated that expansion of the VLDLI lipoproteins in plasma in FCHL was typically associated with the presence of metabolic syndrome. The relationship between plasma VLDL1-TG and plasma TG in FCHL is presented in Figure $2\left(\mathrm{r}^{2}=.835, \mathrm{p}<0.001\right)$.

Table 4. VLDL subclasses in relation to metabolic syndrome. VLDL 1 and VLDL2 were analyzed in controls and FCHL subjects with and without metabolic syndrome.

\begin{tabular}{lccc}
\hline & Controls, Met S (-) & FCHL, Met S $(-)$ & FCHL, Met S (+) \\
\hline N & 13 & 14 & 30 \\
VLDL 1 & & & \\
TG, mmollh & $0.22 \pm 0.11$ & $0.24 \pm 0.13$ & $0.87 \pm 0.61$ \\
Chol, mmol/L & $0.07 \pm 0.06$ & $0.1 \pm 0.07$ & $0.41 \pm 0.43^{*}$ \\
TG/Chol & $3.9(2.6-5.4)^{*}$ & $2.5(1.8-3.2)$ & $2.6(2.0-2.9)$
\end{tabular}

\section{VLDL 2}

TG, mmol/L

$0.2 \pm 0.1$

$0.2 \pm 0.1$

$0.4 \pm 0.2^{*}$

Choll, mmoll

$0.11 \pm 0.06$

$0.15 \pm 0.12$

$0.25 \pm 0.13^{*}$

TG/Chol

$1.8(1.5-2.6)$

$1.7(1.0-2.0)$

$1.4(1.3-2.0)$

VLDL1/VLDL2-TG

$0.7(0.6-11.3)$

$1.1(0.8-1.4)$

$1.6(1.2-3.9)^{*}$

Insulin

$$
3.0 \pm 1.7
$$

$5.3 \pm 1.8$

$10.8 \pm 5.8^{\text {th }}$

${ }^{*} \mathrm{P}<0.05,{ }^{\mathrm{P}} \mathrm{P}<0.01$ and ${ }^{\text {wat }} \mathrm{P}<0.001$ for difference with $\mathrm{FCHL}$ subjects withoul metabolic syndrone (Met $\mathrm{S}$ ) 


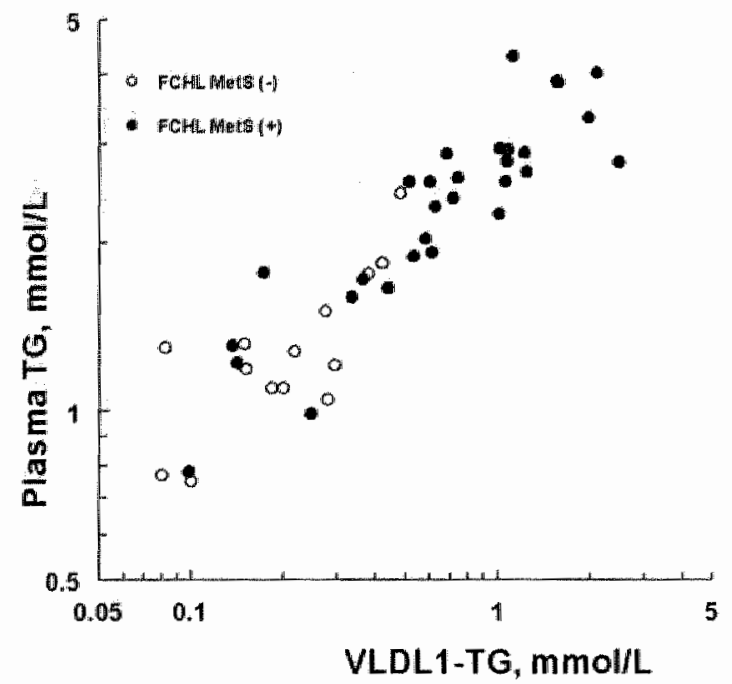

Figure 2.

Relationship between plasma VLDLI-TG and plasma TG in FCHL subjects with or without metabolic syndrome.

Noteworthy, plasma VLDLI-TG concentrations above $0.5 \mathrm{mmol} / \mathrm{t}$ were associated with the presence of metabolic syndrome in all FCHL subjects. Because metabolic syndrome is defined in part by high triglycerides, respectively higher VLDL1-TG levels, we tested the relationship between plasma VLDLL-TG and the presence of ATP III criteria of metabolic syndrome, excluding the criterion hypertriglyceridemia. A positive relationship was observed between VLDLI-TG plasma levels and the presence of other ATP III criteria in FCHL, including increased waist circumference, low HDL cholesterol, and hypertension and impaired fasting glucose (rho $=.39, \mathrm{p}<0.01$, data not presented). In addition, we examined the relationship between VLDL-TG $>0.5 \mathrm{mmol} / \mathrm{L}$, and the presence of at least 3 other ATP III criteria in FCHL, excluding hypertriglyceridemia. Figure 3 shows that FCHL subjects with VLDLL-TG $>0.5$ mmol/L are more likely to have an extended form of the metabolic syndrome $\left(\chi^{2}, p<0.01\right)$. 


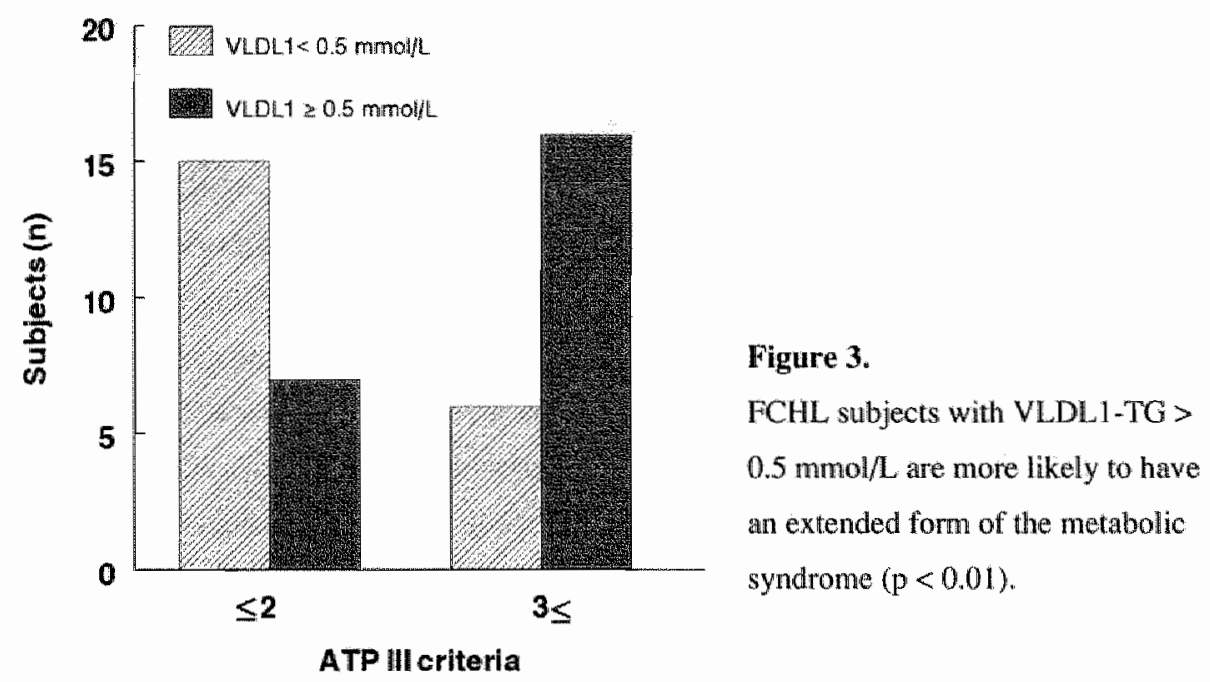

Finally, we evaluated if the relative cholesterol enrichment observed in VLDL in FCHL subjects was related to the presence of metabolic syndrome (Table 4). Significantly lower TG/Chol ratio in VLDL1 was observed in all FCHL subjects, both with $[2.6(2.0-2.9)$, $\mathrm{p}<0.01, \mathrm{p}$ value not shown in Table $]$, and without metabolic syndrome $[2.5(1.8-3.2), \mathrm{p}<$ $0.05]$ compared to controls $[3.9(2.6-5.4)]$. Thus, the presence of low TG/Chol molar ratio, at least in VLDL1, was not related to the presence of the metabolic syndrome, and may thus present an FCHL specific phenomenon.

\section{Discussion}

In the present study, a redistribution of plasma triglycerides towards fractions of lower density (VLDL1) was observed in FCHL subjects compared to controls. This was mainly due to a higher number of VLDLI lipoproteins (VLDLI-Apo B was more than 5-fold higher in FCHL subjects compared to controls, $p<0.01$ ), but not by an increased amount of TG per VLDLI particle, and can thus be regarded as a numerical expansion of plasma VLDLI. lipoproteins in FCHL. Moreover, VLDLI-TG explained more than $80 \%$ of the variability of plasma triglycerides in the study population, whereas the contribution of "FCHL affected 
status" was weak Remarkably, FCHL subjects with expanded VLDLI compartment (VLDL1-TO $>0.5 \mathrm{mmol} / \mathrm{L}$ ) were more likely to thave an extended form of metabolic syndrome, defined as the presence of 3 or more ATP III criteria additional to hypertriglyceridemia.

Hypertriglyceridemia in FCHL was associated with increased numbers of VLDL1 lipoproteins of normal size, rather than VLDL2 lipoproteins, similar to findings in normolipidemic subjects ${ }^{20}$. One explanation for accumulation of VLDLI lipoproteins in plasma in FCHL subjects can be VLDL-Apo B overproduction, as demonstrated in FCHL ${ }^{8}$. Kinetic studies have provided insight on the independent regulation of VLDL1 and VLDL2 production, and their metabolic fate in humans ${ }^{19}$. Hepatic VLDL1 production, but not VLDL2 is inhibited by insulin in healthy subjects ". Thus, increased plasma concentration of VLDL 1 in the face of elevated plasma insulin levels can indicate hepatic insulin resistance. Preferential hepatic production of VLDL1 over VLDL2 can account for the increased ratio of VLDL1-TG over VLDL2-TG in FCHL reported here. Previous studies suggest that alterations in hepatic metabolism contribute to the expression of $\mathrm{FCHL}{ }^{21}$. We have shown that free fatty acid esterification in adipose tissue is insulin resistant in FCHL ${ }^{22}$. This in turn, can lead to an increased flux of fatty acids towards the liver in the postprandial state, contributing to hepatic lipid accumulation. We have reported that fatty liver is present in about $76 \%$ of FCHL subjects ${ }^{16}$. Noteworthy, hepatic lipid accumulation can perturb insulin signaling, i.e. induce hepatic insulin resistance ${ }^{23}$. This may add on to genetic factors that stimulate VLDL1-TG production in FCHL. For instance, propensity to overproduction can relate to a genetic predisposition to lipogenesis de novo in FCHL, mediated by the activity of USF-1, a transcription factor, known to activate fatty acid synthesis ${ }^{24}$. However, the exact biological role of USF-1 remains to be assessed in FCHL.

An alternative explanation for the numerical expansion of plasma VLDL1 lipoproteins may be found in a catabolic defect, independent of overproduction. Decreased activity of the lipoprotein lipase (LPL), which may have a genetic basis ${ }^{6}$ or be the result of insulin resistance ${ }^{25}$, has been reported in about $30 \%$ of FCHL subjects ${ }^{26}$. The VLDL particle itself appears to be a normal substrate for LPL in FCHL, at least in vitro studies ${ }^{27}$. Apparently, overproduction itself can lead to delayed catabolism, because of relative insufficiency of lipolytic pathways ${ }^{28}$. 
Early studies on FCHL report that VLDL lipoproteins tend to be smaller and cholesterol-enriched compared to controls ${ }^{27,29}$. However, in those studies VLDL subclasses were not analyzed separately. In the present study we demonstrated that VLDL1 lipoproteins in FCHL subjects showed normal TG to Apo B ratio, but contained relatively more cholesterol. This finding was independent of the presence or absence of the metabolic syndrome, and can indicate prolonged circulation times ${ }^{30}$, or alternatively, hepatic production of relatively cholesterol enriched VLDL1 lipoproteins. Of interest, Thompson et al. suggest that hepatic VLDL-Apo B production is regulated, at least in part, by cholesterol availability for incorporation in pre-VLDL lipoproteins ${ }^{31}$. Thus, activated cholesterol synthesis in liver can potentially account for production of cholesterol-enriched atherogenic VLDL lipoproteins in FCHL.

Overproduction of VLDL1-TG is known to contribute to increased plasma triglycerides in type 2 DM, yet another disorder with high prevalence of metabolic syndrome 13, 14. However, McEneny et al. reported that VLDL1 lipoproteins in subjects with type 2 DM were particularly larger and lipid rich compared to controls ${ }^{38}$. By contrast, our analyses demonstrated that VLDL1 particle size was not different between subjects with FCHL and controls. Therefore, it is likely that genetic and biochemical differences in hepatic lipid metabolism exist between FCHL and other forms of metabolic syndrome.

FCHL is associated with 2 to 5 -fold increased risk for premature coronary events ${ }^{32}$. The susceptibility to coronary events can be explained at least in part by the high prevalence of metabolic syndrome in $\mathrm{FCHL}^{4}$. The present study identified metabolic syndrome in the majority of hyperlipidemic FCHL subjects (68.2\%) compared to controls ( $27.8 \%$ ), a prevalence quite similar to the reported in literature ${ }^{4}$. Sayle et al. reported that the presence of the metabolic syndrome strongly affected the cardiovascular prognosis among angiographied coronary patients ${ }^{33}$. In subanalysis of the same study, hypertriglyceridemia/low HDL cholesterol significantly predicted the incidence of vascular events in coronary patients with type $2 \mathrm{DM}^{34}$. We showed that particularly FCHL subjects with metabolic syndrome were characterized by high number of plasmaVLDL1 lipoproteins. These observations combined suggest that some of the CAD risk associated with the metabolic syndrome in FCHL can be mediated through VLDLI. 
The present findings can have implications for therapy and prevention of $C A D$ in FCHL. Statins successfully lower plasma lipids, specifically LDL and total cholesterol levels, and are currently the treatment of choice for FCHL. However, Verseyden ef al. reported that VLDLI-Apo B did not lower in FCHL subjects upon statin treatment ${ }^{35}$. Studies of our laboratory showed that although statins lower plasma TG levels (VLDL1-TG), they remained elevated in most PCHL subjects. Furthermore, statin treatment did not ameliorate insulin resistance, as estimated by surrogate markers (Georgieva et al., unpublished observations). Literature suggests that insulin resistance can drive VLDL1 overproduction ${ }^{36}$. This can be the case in our study as well, because the full-blown metabolic syndrome observed in FCHL subjects with bigh plasma VLDL1 suggests a severe degree of insulin resistance ${ }^{33}$. Therefore, targeted treatment of the metabolic syndrome and insulin resistance, e.g. by lifestyle changes, can be beneficial for treatment and CAD prevention at least in some FCHL subjects. It has not been investigated whether pharmacological intervention, i.e. thiazolidinediones can have clinical implications for prevention of $\mathrm{CAD}$ in $\mathrm{FCHL}^{37}$.

In conclusion, hypertriglyceridemia in FCHL is associated with overproduction and/or delayed elimination of VLDL1 lipoproteins of normal size. Numerical expansion of plasma VLDLI lipoproteins is a biochemical characteristic of FCHL subjects with full-blown metabolic syndrome.

\section{Acknowledgements}

We would like to thank the technicians M.G. Robertus-Teunissen and V.M.M.-J. Vermeulen for their excellent work on WLDL subctass separation. Financial support was given by the Deparment of In ternal Medicine. Academic Hospital Mastricht. 


\section{References}

1. Goldstein JL, Hazzard WR, Schrot HG, et al. Hyperlipidemia in coronary heart disease. I. Lipid levels in 500 survivors of myocardial infarction. J Clin Imwest. Jul 1973;52(7):1533-1543.

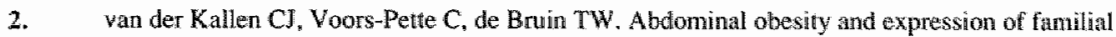
combined hyperlipidemia. Obes Res. Dec 2004;12(12):2054-2061.

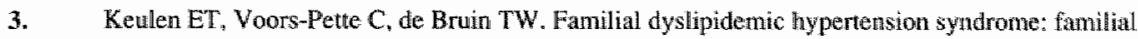
combined hyperlipidemia, and the role of abdominal tat mass. Am J Hypertens. Apr 2001:14(4 Pt 1):357 $\times 363$.

4. Hopkins PN, Heiss G, Ellison RC, et al. Coronary Artery Disease Risk in Familial Combined Hyperlipidemia and Familial Hypertriglyceridemia. A Case-Control Comparison From the National Heart, Lung, and Blood Institute Family Heart Study. Circulation. Jul 72003.

5. Georgieva AM, van Greevenbroek MM, Krauss RM, et al. Subclasses of low-density lipoprofein and very low-density lipoprotein in fanilial combimed lyperlipidemia: relationship to multiple lipoprotein phenotype. Arterioscler Thromb Vasc Biol. Apr 2004;24(4):744-749.

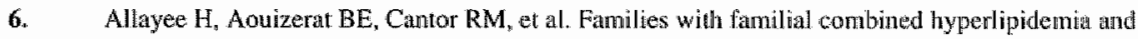
families enriched for coronary artery disease share genetic determinants for the atherogenic lipoprotein phenotype. Am I Hum Genet. Aug 1998;63(2):577-585.

7. Grundy SM, Brewer HB, Jr., Cleeman J, et al. Definition of metabolic syndrome: Report of the National Heart, Lung, and Blood Institute/American Heart Association conference on scientific issues related to definition. Circulation. Jan 27 2004; 109(3):433-438.

8. Venkatesan S, Cullen P, Pacy P, et all. Stable isotopes show a direct relation between VLDL apoB overproduction and serum triglyceride levels and indicate a metabolically and biochemically coherent basis for familial combined hyperlipidemia. Arkerioscler Thromb. Jul 1993; | 13(7):1110-1 11:8.

9. Riches FM, Watts GF, Hua J, et al. Reduction nis wisceral aldipose tissue is associated with improvement in apolipoprotein B- 100 metabolism in obese men. J Clu Endocrinol Meiab. Aug 1999;84(8);28542861.

10. Shelness GS. Sellers JA. Very-low-density lipoprotein assembly and secretion. Carr Opin Lipidol Apr $2001: 12(2): 151-157$.

11. Malmstron R, Packard Cl, Caslake M, ell al. Effects of insul in and acipimox on VLDLL1 and VLDDL2 apolipoprotein B production in normal subjects. Diabetes. May 1998;47(5):779-787.

12. Packard $\mathrm{Cl}$, Shepherd J. Lipoprotein heterogeneigy and apolipoprotein B metabolism. Arferioseder Throust Vase Biol. Dec 1997; 17(12):3542.3556.

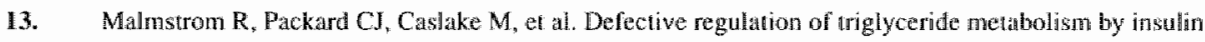
in the liver in NIDDM. Diabetologia. Apr 1997:40(4):454-462.

14. Adiels M, Boren J Caslake MJ, et al. Owerproduction of VLDL1 driven by hyperglycemila is a dominant feature of diabetic dyslipidemia. Arterioscler Thromb Vasc Biol. Atug 2005;25(8): 1697-1703. 
15. Redgrave TO "hobers DC. West CE. Separation of plasma lipoproteins by density-gradient

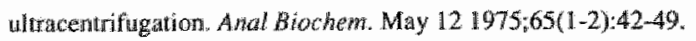

16. de Brum TW, Georgiewa AM, Brouwers MC, et al. Radiological evidence of nonalcobolic fatty liver disease in familial combined byperipidemia. Am I Med. Jun 15 2004; $16(12), 847-849$.

17. Karpe $F$, Hamsten A Detemination of apolipoproteins B-48 and B-100 in triglyceride-rich lipopreteins by alkallycical SDS.PACE J Lipid Res. Jul 1994;35(7):1311-1317.

18. Catpentier A, Patterson BW, Lung N, et al. Sensitwity to acute insulin-mediated stppression of plisma free faty acids is not a determinant of fasting VLDL triglyceride secretion in healthy humans.

Diabetes. Jun 2002;51(6):1867-1875.

19. Bemeis KK. Krtuss RM. Metabolic origins and clinical significance of LDL heterogeneity. $/ 2$ Lipid Res. $\operatorname{Sep} 2002 ; 43(9): 1363-1379$.

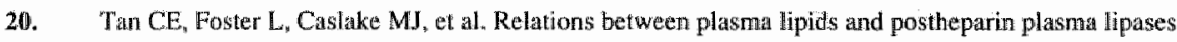
and VLDL and LDL subfracion patherns in normolipemic men and wonen. Arterioscler Thromb Vasc Biol. Nov 1905:15(11):1839-1848.

21. Castro Cabezas $M$, de Bruan TW, de Valk HW, el al. Impared fatly acid netabolism in familial combined hyperlipicienia. A mechanism associating hepatic apolipoprotein $B$ overproduction and insulin tesistance. JChin Irwest. Jull 1993;92(1):160-168.

22. van der Kallen $\mathrm{CJ}$, Voors Pette $\mathrm{C}$, Bouwman $\mathrm{FG}$, et al. Evidence of insulin resistant lipid metabolism in adipose tissue in familial combined hyperlipidemia, but not type 2 dinbetes nellius. Atherasclevesis. Oci $2002 ; 164(2): 337-346$

23. Samuel VT, Liu $Z X, Q u X_{*}$ et al. Mechamism of hepatic insulin resistatuce in non-alcoholic fatry liver disease. J Biol Chem. May 272004.

24. Pajukantio P. Lilja HE, Sinsheimer IS, et all. Familial combined hyperlipidemia is associated with upstream tratrgcription factor 1 (USF1). Nat Genet. Apt 2004;35(4):371-376.

25. Mead JR, Irvine SA, Ramji DP. Lipoprotein lipase: structure, function, regulation, and role in disense. J Mol Med. Dec 2002,80(12):753-769.

26. Babiratk SP, Brown BG, Brunzell JD. Fatmilial combined hypertipidemian and abaormad lipoprotein Lipase. Ancrionclen Thomb. Oct 1992;12(10):1176m:1183.

27. was Barlingen HH, Kock LA, de Man FH, en al, In vitro lipolysis of human VLDL. effect of different VLOL ${ }_{\text {wa }}$ compositions in nomolipidemian familial combined hyperdipidemia and familial hepetriglyceridenia. Arherosderosis. Mar 1996;21(1):75-84.

26. Bjorkegrea $\mathrm{J}$, Packard $C \mathrm{I}$, Hamsten A, et al. Accumulation of large very low density lipoproten in plasma during intravenous infusion of a chylonncron-like triglyceride emul sion reflects competition for a conmon lipolytic pathway. Jipid Res. Jan 1996,37(1):76-86.

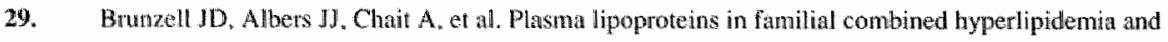
monogentc fambial hypentrigheeridemua. J Lipid Res. Fab 1983;24(2):147-155.

30. Guerin M, Egger P. Soutant C, at at. Cholesteryl ester flux from HDL to VLDL- I is preferentially enhanced in rype IIB hyperlipidema in the postprandial state. J Lipid Res. Oc 2002;43(10):1652-1660. 
31. Thompson GR, Naoumova RP, Watts GF. Role of cholesterol in regulating apolipoprotein B gecretion by the liwer. I Lipid Res. Mar 1996;37(3):439-447.

32. Voors-Pette $C$, de Bruin TW. Excess coronary heart disease in Familial Combined Hyperlipidemial in relation to genetic factors and central obesity. Anderosclerosis. Aug $2001,157(2): 481-489$.

33. Saely $\mathrm{CH}$, Aczel $\mathrm{S}$, Marte $\mathrm{T}$, et al. The Metabolic Syndrome, Insulin Resistance, and Cardiovascular Risk in Diabetic and Nondiabetic Patients. I Clin Endocrinol Metab. Aug 9005.

34. Drexel H, Aczel S, Marte $\mathrm{T}$, et al. Is atherosclerosis in diabetes and impaired fasting glucose driven by elevated LDL cholesterol or by decreased HDL cholesterol? Diaberes Came Jan $2005,28(1): 1014107$.

35. Verseyden $C$, Meijssen S, Cabezas MC. Effects of atorwastatin on fasting plasma and marginated apolipoproteins B48 and B100 in large, triglyceride-rich lipoproteins in familial combined hyperlipidemia. I Chnt Endocrinol Metab. Oct 2004;89(10):5021-5029.

36. Watts GF, Chan DC, Barrett PH, et al. Effect of a statin on hepattic apolipoprotein B-100 secretion and plasma campesterol levells in the metabolic syndrome. Int J Obes Relat Metal Disord. Jul $2003 ; 27(7): 862-865$

37. Yki-Jarvinen $\mathrm{H}$. Thiazolidinediones. N Engl J Med. Sep $92004 ; 351111) 1106-1118$.

38. McEneny J, O'Kane MJ, Moles KW, et al. Very low density lipoprotein subfractions in Type II diabetes mellitus: alterations in composition and susceptibility to oxidation. Diaberologia. Apr $2000 ; 43(4): 485$ 493. 
Chapter 6

Lipitor, Inflammation and Gene Expression in Adipose Tissue (LIGEAT) in Familial Combined Hyperlipidemia.

I. Effect on plasma lipid and lipoprotein profile, and insulin resistance.

Anna M. Georgieva, Martijn C.G.J. Brouwers, Carla J.H. van der Kallen, Marleen M.J. van Greevenbroek, Tjerk W.A. de Bruin 


\section{Summary of Study Design}

Differential gene expression analysis has indicated an inflammatory process in subcutaneous adipose tissue in FCHL subjects compared to controls. This process can represent a response to hyperlipidemia in FCHL. To test this hypothesis, we treated 12 hyperlipidemic FCHL subjects with a statin (atorvastatin). We performed a subcutaneous adipose tissue biopsy in each subject before $(t=0)$ and after 8 weeks of treatment $(t=8)$. Here we present the changes in plasma lipid and lipoprotein profile induced by statin therapy, in combination with plasma inflammatory markers and surrogate markers for insulin resistance. The gene expression analysis can help us identify pathways involved in adipocyte adaptation to hyperlipidemia in FCHL. This latter data however is beyond the scope of this thesis. 


\section{Introduction}

Familial Combined Hyperlipidemia (FCHL) was delineated as a genetic disorder of lipid metabolism almost 3 decades ago'. Affected FCHL subjects show a complex insulin resistant phenotype and show a number of abnormalities in lipid metabolism: hypercholesterolemia (elevated total and LDL cholesterol) and/or hypertriglyceridemia,

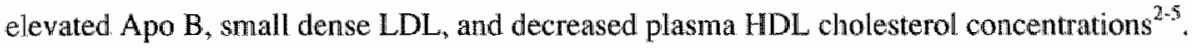
FCHL subjects have a higher risk of premature coronary artery disease (CAD) than the population, which can be related at least in part to the higher prevalence of the metabolic syndrome in FCHL 6.7 .

Recently, our laboratory demonstrated abnormalities in gene expression in subcutaneous adipose tissue from FCHL subjects, imvolving cell cycle genes, insulith resistance genes and inflammatory genes, including TNF-alpha (13 fold elevated vs. controls) and IL- $6^{8}$. Of interest, these adipokines have been associated with systemic insulin resistance and $C A D^{9}$. The observed changes in gene expression can result from a primary genetic defect, or alternatively represent an adaptation to hyperlipidemia, or a combination of both. In the case of adaptation, lipid-lowering with a pharmaceutical compound can result in improved organ function, which in turn can lead to a reduction in systemic inflammation and insulin resistance.

Overproduction of Apo B-containing lipoproteins can explain, at least in part, hyperlipidemia in FCHL ${ }^{10}$. Delayed clearance of VLDL and VLDL remnants contributes to hyperlipidemia as well, as shown in hypenriglyceridennic FCHL subjects" "The majority of hypertriglyceridemic FCHL subjects have elevated plasma insulin, a surrogate marker for insulin resistance $2,12,13$. Therefore, it is possible that insulin resistance induces alteration in the metabolism of Apo B-containing lipoproteins that results in hypertriglyceridemia. Alternatively, insulin resistance can ensue, at least in part, from elevated triglyceride-rich lipoprotein levels. Again, lipid lowering with a medication can help us evaluate whether elevated plasma insulin is secondary to hypertriglyceridemia in FCHL.

This study was designed to assess if lipid lowering with atorvastatin has a beneficial effect on subcutaneous adipose tissue function of FCHL subjects. In addition, changes in concentration and composition of Apo B-containing lipoproteins, in combination with 
surrogate markers for insulin resistance and inflammatory markers have been evaluated. For that purpose, 12 hyperlipidemic FCHL subjects were treated with atorvastatin (Lipitor(B) $40 \mathrm{mg}$ dally, as monotherapy). The following parameters were evaluated before and after 8 weeks of treatment: 1) plasma lipid levels, 2) indirect markers of insulin resistance 3) plasma levels of proinflammatory markers. Here we present in detail the changes in lipoprotein subclasses and metabolic profile, induced by atorvastatin therapy.

\section{Methods}

Twelve unrelated FCHL subjects were recruited through the Lipid Clinic of the Maastricht University Hospital. Briefly, FCHL subjects had: 1) primary hyperlipidemia with varying phenotypic expression, including (untreated) fasting plasma cholesterol $>6.5 \mathrm{mmol} / 1$ and/or fasting plasma triglyceride concentration $>2.3 \mathrm{mmol} / \mathrm{l} ; 2$ ) they were known with premature CAD (before the age of 60), or had a positive family history of such; 3 ) had at least one other first-degree relative with a different lipoprotein phenotype. Nine FCHL subjects represented well-defined FCHL families. Three subjects presented with an FCHL phenotype (primary hyperlipidemia, and a positive history of premature CAD), but the diagnosis could not be established in a family, because of nuclear families. The Human Investigation Review Committee of the Academic Hospital Maastricht approved the study protocol. All subjects gave informed consent.

Subjects were asked to stop any lipid-lowering medication 4 -week prior to study (5 subjects had been treated with a statin, 3 subjects with a combination therapy of a statin and a fibrate). One subject was treated with Avandiat, $4 \mathrm{mg} /$ day and this medication was not discontinued. Before inclusion, after the washout period, 6 subjects presented with combined Iyperlipidemia (Fredrickson phenotype IIB, LDL cholesterol > $4.2 \mathrm{nmol} / \mathrm{L}$, triglycerides $>2.3 \mathrm{mmol} / \mathrm{L}$ ), and 6 subjects with isolated hyperlipidemia (Fredrickson type IV, LDL cholesterol $<4.2 \mathrm{mmol} / \mathrm{L}$, triglycerides $>2.3 \mathrm{mmol} / \mathrm{l}$. Two subjects presented with highly elevated plasma triglycerides of $19.0 \mathrm{mmol} / \mathrm{L}$ and $22.7 \mathrm{mmol} / \mathrm{L}$ (Fredrickson type IV). Atorvastatin $40 \mathrm{mg}$ at bedime was prescribed for 8 weeks as monotherapy. 
At zero and eight weeks after start of treatment $(t=8)$, venous blood was drawn in precooled EDTA ( $1 \mathrm{mg} / \mathrm{ml})$ tubes after an overnight fast $(12-14 \mathrm{~h})$, for the following analyses: 1$)$ lipid analyses - total cholesterol, HDL cholesterol, plasma triglycerides, Apo $B$; 2) surrogate markers of insulin resistance - insulin, glucose, FFA; 3) plasma inflammatory markers. For gene expression analysis, abdominal subcutaneous fat biopsies were obtained before $(t=0)$, and after 8 weeks $(t=8)$, of atorwastatin treatment.

VLDL1 and VLDL2 were separated from plasma VLDL $(<1.006 \mathrm{~g} / \mathrm{ml}$ on a $\| .006$ * $1.250 \mathrm{~g} / \mathrm{ml} \mathrm{KBr}$ density gradient) by density gradient ultracentrifugation at $160000 \mathrm{~g}$ for 2.5 hours at $4^{\circ} \mathrm{C}$ in SW40 Ti rotor, as described ${ }^{2}$. Measurements of triglycerides and cholesterol in the subfractions were done by standard laboratory techniques. Concentrations of VLDLI and VLDL2 Apo B was measured by gel electrophoresis according to the method of Karpe et al. $^{14}$,

\section{Statistical analyses}

Wilcoxon's nonparametric test for two related samples was used to evaluate the effect of treatment. Correlations were assessed by Spearman rank correlation. In all analyses, the statistical package SSPS 11.0 (SSPS Inc.) was used.

\section{Results}

\section{Effect of therapy on plasma lipids and markers of insulin resistance}

All subjects completed the study. One subject completed the study prematurely at week 6 because of complains of insomnia, constant fatigue and headache. Characteristics of the study population and results are presented in Table 1. Of note, all subjects were viscerally obese and fulfilled the criteria for metabolic syndrome, defined according to Adult treatment panel III of the NCEP (ATP III) $)^{15}$.

At 8 weeks of treatment, Atorvastatin, $40 \mathrm{mg} / \mathrm{d}$ as monotherapy induced significant reduction in fasting total cholesterol $(-41 \%)$, triglycerides $(-38 \%)$, Apo B $(-26 \%)$, as well as significant increase in HDL-cholesterol (19\%). The change in triglycerides was significantly correlated with triglycerides at baseline ( $R h o=-63, p=0.03$ ). However, no changes were 
seen in plasma insulin, glucose. FFA. Moreover, metabolic syndrome was still present in all subjects at the end of study:

We did not observe significant changes in ALT with atorvastatin treatment. However, nine patients showed a reduction in ALT levels, suggesting an improved liver function.

Table 1. Characteristics of study group. Effect of 8 weeks of Atorvastatin $40 \mathrm{mg}$ per day, monotherapy

\begin{tabular}{|c|c|c|c|c|}
\hline & Before treatment & After treatment & $P$ & $\%$ Change \\
\hline \multicolumn{5}{|l|}{ Descriptives } \\
\hline Number $(M / F)$ & $12(7 / 5)$ & & & \\
\hline Age & $52(34-65)$ & & & \\
\hline BMI kg/ $/ \mathrm{m}^{2}$ & $29.0(26.9-34.1)$ & & & \\
\hline $\mathrm{SBP}, \mathrm{Hgmm}$ & $136(107-161)$ & $132(107-178)$ & NS & $-3.6 \%$ \\
\hline DBP, Higmm & $85(72-110)$ & $85(66-107)$ & NS & $-1.8 \%$ \\
\hline \multicolumn{5}{|l|}{ LLlpid proflle } \\
\hline $\mathrm{TC}, \mathrm{mmoln}$ & B. $1(5.4-12.1)$ & $4.7(3.4-8.4)$ & .002 & $-41 \%$ \\
\hline Apo B, gh & $1.2(0.8-1.6)$ & $0.8(0.6-1.3)$ & .003 & $-26 \%$ \\
\hline$T G, m m o l / l$ & $3.8(2.3-22.7)$ & $2.7(0.9-5.1)$ & .008 & $-38 \%$ \\
\hline HDL-chol, mmol/m & $0.7(0.4-1.3)$ & $0.8(0.4-1.2)$ & .028 & $+19 \%$ \\
\hline \multicolumn{5}{|l|}{ Markers of Inswlin } \\
\hline \multicolumn{5}{|l|}{ Besilstance } \\
\hline HOMA & $3.0(1.1-9.8)$ & $2.8(0.8-9.6)$ & NS & $+3 \%$ \\
\hline$\|$ nsulin $\mu N / m \mid$ & $11.1(4.7-35.6)$ & $11.7(3.6-34.7)$ & $\mathbb{N S}$ & $+5 \%$ \\
\hline FFA, mmoln & $0.4(0.06-0.9)$ & $0.3(0.06-0.6)$ & NS & $-3 \%$ \\
\hline \multicolumn{5}{|l|}{ Llver Function } \\
\hline ALT U/I & $31.4(11.4-118.5)$ & $26.8(12.5-85.4)$ & NS & $-17.0 \%$ \\
\hline
\end{tabular}

Data are presetved as Median (range)

Abbreviations: $\mathrm{M} / \mathrm{F}=$ male/female; $\mathrm{BMI}=$ body-mass index: $\mathrm{SBP}=$ systotic blood pressure; $\mathrm{DBP}=$ diastolic blood pressure; $T C=$ total cholesterol; HDL-chol $=$ high density lipoprotein cholesterol: HOMA $=$ honeostatic model assessment index; $\mathrm{FFA}=$ free fary acids; $\mathrm{ALT}=$ alanine amino transferase 


\section{Effect of therapy on Apo B-containing lipoproteins}

Changes in Apo B containing lipoprotein fractions are presented in Table 2.

Table 2. Changes in lipoprotein profille induced by atorvastatin monotherapy (40 $\mathrm{mg}$ per day).

\begin{tabular}{|c|c|c|c|c|}
\hline & Before treatment & After treatment & $\mathbf{P}$ & $\% \%$ Change \\
\hline \multicolumn{5}{|l|}{ VLDLI } \\
\hline $\mathrm{TG}_{1} \mathrm{mmol} / \mathrm{l}$ & $1.4(0.6-15.0)$ & $0.8(0.1-2.1)$ & .003 & $-52 \%$ \\
\hline $\mathrm{Chol}, \mathrm{mmol} / \mathrm{I}$ & $1.0(0.3-6.4)$ & $0.5(0.04-1.3)$ & .004 & $-77 \%$ \\
\hline Apo B mg/l & $67.7(16.5-285.7)$ & $27.2(0.8-88.6)$ & .002 & $-58 \%$ \\
\hline \multicolumn{5}{|l|}{ VLDL2 } \\
\hline $\mathrm{TG}, \mathrm{mmol} / \mathrm{l}$ & $0.8(0.4-3.7)$ & $0.7(0.3-1.6)$ & .03 & $-25 \%$ \\
\hline Chol, $\mathrm{mmol} / / \mathrm{l}$ & $0.5(0.2-2.9)$ & $0.4(0.05-1.0)$ & .02 & $-40 \%$ \\
\hline Apo B mg/l & $68.4(42.5-216.1)$ & $47.7(13.3-130.7)$ & .003 & $-26 \%$ \\
\hline VLDL1NLDL? & $1.8(0.9 \cdot 9.6)$ & $1.6(0.3-1.9)$ & .02 & $-38 \%$ \\
\hline \multicolumn{5}{|l|}{ IDL } \\
\hline TG, mmol/ & $0.14(0.1-0.24)$ & $0.12(0.06-0.2)$ & .003 & $-25 \%$ \\
\hline $\mathrm{Chol}_{\text {" }} \mathrm{mmol} / \mathrm{l}$ & $0.55(0.27-0.94)$ & $0.33(0.09-0.85)$ & .004 & $-38 \%$ \\
\hline \multicolumn{5}{|l|}{ LDL } \\
\hline $\mathrm{TG}, \mathrm{mmol} / \mathrm{l}$ & $0.25(0.16-0.39)$ & $0.17(0.1-0.33)$ & .002 & $-29 \%$ \\
\hline $\mathrm{Chol}_{\text {" } \mathrm{mmol} / \mathrm{l}}$ & $3.69(1.34-6.11)$ & $1.90(1.21-5.07)$ & .003 & $-37 \%$ \\
\hline Apo B, gnl & $1.2(0.6-1.6)$ & $0.7(0.6-1.3)$ & .002 & $-37 \%$ \\
\hline
\end{tabular}

Data are presented as Median (ramge)

Significant decrease of TG and cholesterol were observed in all fractions. The major decrease, was observed for VLDLI fraction: VLDL I-TG lowered by $52 \%$, VLDL1 -Chol by $77 \%$, and VLDL1 Apo B by 58\% ( $<<0.01$ for all). For VLDL2, VLDL2-TG lowered by 25 $\%$, VLDL2 Chol by $40 \%(\mathrm{p}<0.05$ for both), and VLDL2 Apo B by $26 \%$ (p<0.01). This resulted in a significantly lower ratio of VLDL I-TG over VLDL2-TG, which indicated 
improvement in fasting plasma TG distribution. IDL-TG lowered by $25 \%$, IDL. Chol by $37 \%$ ( $p<0.01$ for both). A similar reduction was obserwed in the $\mathrm{LDL}$ fraction. In addition, we did not see changes in the LDL/Apo B ratio, an estimation of LDL size.

No relationship was observed between changes in lipoprotein levels and surrogate markers for insulin resistance, or lipid variables, or ALT.

\section{Effect of therapy on proinflammatory markers}

The most dramatic change after 8 weeks of treatment was the significant reduction of plasma PAI-1 levels $(-18 \%, \mathrm{p}<0.05)$ (Table 3 ). The reduction in CRP levels by $21 \%$ approached statistical significance. However, the reduction in other proinflammatory markers, such ICAM-1, TNF- $\alpha$ R1 and TNF- $\alpha$ R2 levels was minimal and did not reach statistical significance.

Again, no relationship was observed between changes in PAI-1 or CRP and surrogate markers for insulin resistance.

Table 3. Changes in prointlammatory markers induced by atorvastatin monotherapy.

\begin{tabular}{lcccc}
\hline & Before treatment & After treatment & $P$ & $\%$ Change \\
\hline CRP, ng/ml & $4.0(1.7-8.7)$ & $3.0(1.2-7.6)$ & .099 & $-21 \%$ \\
PAl-1, ng/ml & $20.1(3.4-36.9)$ & $15.6(4.6-24.9)$ & .012 & $-18 \%$ \\
ICAM-1, ng/ml & $72.5(38.5-126.4)$ & $70.2(36.5-121.8)$ & NS & $-5 \%$ \\
TNF - a R1, ng/ml & $1.6(1.2-2.7)$ & $1.6(1.0-2.2)$ & .087 & $-7 \%$ \\
TNF-a R2, ng/ml & $1.8(1.2-2.7)$ & $2.0(0.7-2.5)$ & NS & $-5 \%$ \\
\hline Data are presented is Median (range) & &
\end{tabular}

Dath are presented as Median (range)

Abbreviations: CRP $=$ C-reactive protein $\mathrm{PAl}-1=$ plasminogen activator inhibitor $\mathbf{I}$ ICAM-I = intracellular adlucsion molecule $1 ;$ TNF-a $R 1=$ tuninor necrosis factor 1 receptor 1 ; TNF $\mathrm{Q}$ R2 $=$ tumor necrosis factor 1 receptor 2 


\section{Discussion}

Treatment with atorvastatin $40 \mathrm{mg}$ per day at bedtime for 8 weeks effectively lowered plasma lipids in hypertriglyceridemic FCHL subjects, without concomitant changes in surrogate markers for insulin resistance (plasma insulin, HOMA or plasma FFA concentrations). The main changes in Apo B-containing lipoprotein fractions were observed in VLDL1. However, plasma triglycerides remained elevated ( $>2 \mathrm{mmol} / \mathrm{L}$ ) on therapy in most subjects.

Statins effectively lowered plasma Apo B-containing lipoproteins. Several mechanisms are known to mediate this effect in hypertriglyceridemic subjects, the most important of all being increased hepatic uptake via the LDL receptor. Statins inhibit cholesterol synthesis, primarily in liver, and thereby induce upregulation of hepatic LDL receptors. Enhanced hepatic clearance of VLDL-Apo B lipoproteins by $58 \%$ has been observed in subjects with the metabolic syndrome ${ }^{16,17}$. In addition, increased activity of L.PL contributes to faster conversion of VLDL1 to VLDL2, and thereby promotes VLDL. clearance ${ }^{17}$. Indeed, we saw an improvement in the VLDL1/NLDL2TG ratio, which can at least in part be related to increased LPL activity. Statins decrease the production rate of VLDL-Apo B in healthy subjects, but this mechanism does not operate in subjects with the metabolic syndrome ${ }^{16}$. Insulin resistance in liver seems to mediate overproduction of VLDLApo $\mathbf{B}$ in the latter subjects ${ }^{16,17}$. In the present study treatment with atorvastatin did not ameliorate insulin resistance in FCHL subjects, as estimated by surrogate markers (HOMA, of fasting insulin). It is likely that overproduction in these FCHL subjects resulted from relative insulin resistance in liver as well, because treatment with statins improved but did not normalize plasma $\mathrm{TG}$ llevels ${ }^{18}$.

An atherogenic cluster of elevated plasma TG, low HDL and sd LDL is a common finding in FCHL ${ }^{2}$. Atorvastatin treatment resulted in $19 \%$ increase in $\mathrm{HDL}$, but values remained low, at least in men ( HDL-cholesterol $<1.00 \mathrm{mmol} / 1)^{15}$. Several groups have reported persistence of sd LDL in FCHL upon statin treatment ${ }^{19,20}$. In agreement, we did not see changes in the LDL/Apo B ratio, which can be used for estimation of LDL size. There are at least two mechanisms that can explain the persistence of the atherogenic prontle on statin therapy. First, statins lower VLDL1 levels, but most likely do not resolve overproduction ${ }^{16}$. 
An expansion of the VLDL 1 lipoproteins in plasma mediates low HDL levels and formation of sd LDL" ${ }^{2 !}$. This process involves neutral lipid exchange via the cholesterol ester transfer protein (CETP) ${ }^{22}$. Second, increased activity of hepatic lipase (HL) can contribute to formation of sd LDL and low HDL cholesterol as well. HL activity is increased in insulin resistance ${ }^{23}$ and has been implicated as a modifier gene in the expression of $\mathrm{FCHL}^{24}$. Moreover, changes in HL activity have not been observed upon atorvastatin therapy in $\mathrm{FCHL}$.

The major changes in Apo B containing proteins affected the VLDLl fraction, where both lipid and apolipoprotein content decreased by more than $50 \%$. This is in contrast to the findings of Castro Cabezas et al., who did not observe changes in VLDL1- TG and VLDL1apo $\mathrm{B}$ in FCHL subjects upon atorvastatin treatment ${ }^{26}$." The reason for this inconsistence can relate to higher plasma TG on start of atorvastatin therapy in our study. Current insight suggests that the response to statin therapy is phenotype dependent: the higher basal triglycerides (above $1.5 \mathrm{mmol} / \mathrm{L}$ ), the better the response ${ }^{17}$.

Elevated leveis of proinflammatory markers and prothrombotic markers, in particular CRP and PAI- 1 indicate an increased risk for $\mathrm{CAD}^{27-29}$. PAI-1 lowered significantly and there was a tendency for reduction in CRP upon statin treatment. Some but not all studies report changes in PAI- 1 levels ${ }^{30,31}$. The reduction in PAI-1 and CRP was not associated with changes in lipid levels, in agreement with previous reports in literature ${ }^{30}$. Because of these pleiotropic actions, statin therapy can be important for prevention of acute cardiac events in FCHL patients. Moreover large studies have demonstrated the antiatherogenic effects of statin therapy in the long term ${ }^{34}$.

The reduction in PAI- $\$$ observed in the study can be related to improved endothelial function $^{31.32}$, or alternatively reduced inflammatory response in subcutaneous fat tissue, or a combination of both. Additional mechanism that can explain reduction of PAI- 1 with statin therapy in FCHL is improvement in liver function. Fatty liver can be an important source of PAI-I in hypertriglyceridemic FCHL subjects ${ }^{33}$. Noteworthy, we observed reduction in ALT levels after 8 weeks of treatment in the majority of the study subjects, indicating an improved liver function. This observation suggests that statin therapy can be potentially beneficial in the treatment of non-alcoholic fatty liver disease in FCHL. However, elevation in liver transaminases is a known side-effect of statin therapy, and therefore caution is required. 
Importantly, the increased risk of CAD in the metabolic syndrome is related to a clustering of metabolic abnormalities, rather than a single factor ${ }^{15}$. Statin treatment did not resolve the insulin resistance, estimated as surrogate markers, and dyslipidemia in FCHL. Therefore, therapies that target insulin resistance are potentially beneficial in FCHL, such as thiazolidinediones (TZD) ${ }^{35}$ or life style changes ${ }^{36}$. However, data on the effect of TZD on CAD is still. not available.

In conclusion, treatment with atorvastatin effectively lowered plasma lipids, but did not resolve insulin resistance and the atherogenic lipoprotein profile of hypertriglyceridemic FCHL patients. Atorvastatin reduced levels of cardiovascular risk markers, particularly PAI-1 and CRP. Noteworthy, no relationship was observed between changes in PAI-1, or CRP and lipid wariables. Because of these pleiotropic effects, statins can be important in treatment of FCHL, especially CAD prevention. Potentially, hypertriglyceridemic FCHL patients might have an additional benefit from therapies that target insulin resistance.

\section{Acknowledgements}

This study was supported by a grant from Pfizer Netherlands. 


\section{References}

1. Goldstein $\mathrm{JL}_{s}$ Schrot HG, Hazzard $\mathrm{WR}_{\mathrm{p}}$ et al. Hyperlipidemia in coronary heart disease. II. Genetic analysis of lipid lewels in 176 fanilies and delineation of a new inherited disorder, combined hyperlipidemia. J Chin Invest. Jul 1973:52(7): 1544-1568.

2. Georgiewa $\mathrm{AM}_{2}$ Van Greevenbroek $\mathrm{MM}_{\mathrm{v}}$ Krauss RM, et al. Subclasses of Low-Density Lipoprotein and Very Low-Density Lipoprotein in Familial Combined Hyperlípidemia: Relationship to Multiple Lipoprotein Phenotype. Arteriascler Thromb Vasc Biol Jan 292004.

3. Wan der Kallen Cl, Voors-Pette C, de Bruin TW. Abdominal obesity and expression of familial combined hyperlipidemia. Obes Res. Dec 2004;12(12):2054-2061.

4. Purnell JQ, Kahn SE, Schwartz RS, et al. Relationship of insulin sensitivity and ApoB levels to intraabdlominal fat in subjects with farnilial combined hyperlipidemia. Arterioscler Thromb Vasc Biol. Apr 2001;21(4):567-572.

5. Aitman TJ, Godsland IF, Farren B, el al. Defects of insulin action on fatty acid and carbohydrate metabolism in familial combined hyperlipidemia. Arterioscler Thromb Vasc Biol. Apr 1997;17(4):748. 754.

6. Hopkins PN, Heiss G, Ellison RC, et al. Coronary Artery Disease Risk in Familial Combined Hyperlipidemia and Familial Hypertriglyceridemia. A Case-Comtrol Comparison From the National Heart, Lung, and Blood Institute Family Heart Study. Circulation. Jul 72003.

7. Voors-Pette $\mathrm{C}_{\mathrm{v}}$ de Bruin TW. Excess coronary heart disease in Familial Combined Hyperlipidemia, in relation to genetic factors and central obesity. Atherosclerosis. Aug 2001:157(2):48 1-489.

8. Eurlings PM, Van Der Kallen CJ, Geurts JM, et al. Identification of differentially expressed genes in subcutaneous adipose tissue from subjects with familial combined hyperlipidemia. J Lipid Res. Jun $2002: 43(6) ; 930-935$.

9. Libby P. Inflammation in atherosclerosis. Nature. Dec 19-26 2002;420(6917)"868-874.

10. Venkatessin $S$, Cullen $P$, Pacy $P$. et al. Stable isotopess show a direct relation between VLDL apoB overproduction and serum triglyceride levels and indicate a metabolically and biochemically coherent basis for fanilial combined hyperlipidemia. Arterioscler Thromb. Jul 1993;13(7):111041118.

11. Castro Cabezas $M$, de Bruin TW, de Walk HW, et al. Impaired fatty acid metabolism in familial combined hyperlipidemia. A mechanissn associating hepatic apolipoprotejs B overproduction and insulin resistance. J Clint Imvest. Jul 1993;92(1):160-168.

12. Pihlajamaki J, Karjalainen L, Karhapa P, et at. Impaared ftee fatty acid suppression during hyperinsulinemia is a characteristic finding in fanilial combined hyperlipidemia, but insulin resistance is observed only in hypertriglyceridemic patients. Arteriowcler Thromb Vaxe Biol. Jan 2000;20(1): 164. 170.

13. Vakkilainen J, Porkka KV. Nuotio $\mathrm{I}$, et al. Glucose intolerance in familial combined hyperlipidaemia. EUFAM study group. Eur J Clin Invest. Jan 1998;28(1):24-32. 
14. Karpe $F$, Hamsten A. Determination of apolipoproteins $B-48$ and $B-100$ in triglyceride rich lipoproteins by analytical SDS-PAGE. I Lipid Res. Jul 1994:3507):1311-1317.

15. Grundy SM, Brewer HB, JT., Cleeman II, et al. Definition of metabolic syndrome: Report of the National Heart, Lung, and Blood Institute/American Heart Association conference on scientific issues related to definition. Circularion. Jan $272004 ; 109(3) 433-438$

16. Watts GF, Chan DC, Barrett. PH, et al. Effect of a statin on hepatic apolipoprotein B-100 secretion and plasma campesterol levels in the metabolic syndrome. In J Obes Relat Mefab Disord. Jil $2003 ; 27(7): 862-865$

17. Caslake MJ, Packard CJ. Phenotypes, genotypes and response to statin therapy. Carr Opin Lipidol. Atig $2004 ; 15(4): 387-392$.

18. Castro Cabezas M, Erkelens DW, Kock LA et al. Postprandial apolipoprotein B 100 and B48 metabolism in familial combined byperlipidaemia before and after reduction of fasting plasm triglycerides. Eur J Clin Imvest. Oci 1994:24(10):669-678.

19. Bredie SI, de Bruin TWV, Demacker PN, et al. Comparison of genfibrozil versus simvalstatin in familial combined hyperlipidemia and effects on apolipoprotein-B-containing lipoproteins, low-density lipoprotein subfraction profile, and low-density lipoprotein oxidizability. Am J Cardial. Feb 15 $1995 ; 75(5): 348-353$.

20. Franceschini $G$, Cassinoti $M$, Vecchio $G$, et al. Pravastatin effectively lowers LDL cholesterol in familial combined hyperlipidemia without changing LDL subclass pattern. Arterioncler Thromb. Oct $1994: 14(10): 1569-1575$.

21. Bemeis KK. Krauss RM. Metabolic origins and clinical significance of LDL heterogeneity. J Liphd Res. Sep 2002:43(9):1363-1379.

22. Guerin M, Lassel TS, Le Goff W, et al. Action of atorvastatin in combined hypertipidemia: preferential reduction of cholesteryl ester transfer from HDL to VLDLL particles. Arterioscler Thromb Vasc Bial. $\operatorname{Jan} 2000 ; 20(1): 189-197$.

23. Gill JM, Brown JC. Bedford D, et al. Hepatic production of VLDLI bat aot VLDL2 is nelated io insulin resulstance in normoglycaemic middle-aged subjects. Aherosclerosis Sep 2004;176(1):49-56.

24. de Bruin TW, Maily F, van Barlingen HH, et al. Lipoprotein lipase gene nulations D9N and N291S in four pedigrees with familial combined hyperlipidaemia. Eur J Clin Invest. Aug 1996;26(8):631-639.

25. Cabezas MC, de Bruira TW, Jansen $H$, el al. Impaired chylomicron remnant clearance in familal combined hyperlipidemian. Arterioscles Thomb. Jun 1993;13(6):804-814.

26. Castro Cabezas M, Verseyden C. Meijsen S, et ail. Faffects of atorvastatim on the clearance of triglyceride-rich lipoproteins in familial combined hyperlipidemia. I Clin Endocrimol Metab. Dec 2004;89(12):5972-5980.

27. Pai $J K$, Pischon $\mathrm{T}_{\text {" }} \mathrm{Ma} \mathrm{J}_{4}$ et al. Inflammatory markers and the risk of coronary heart disease irn men and women. N Engl I Med. Dec 16 2004;351(25);2599-2610.

28. Thogersen AM, Jansson JH, Boman K, et al. High plasminogen activator inhibitor and tissue plasminogen actiwator levels in plasma precede a first acute myocardial infarction in both men and 
woment evidence for the fibrinolytic system as an independent primary risk factor. Circthation. Now 24 $1998 ; 98(21): 2241-2247$

29. Anand SS, YiQ. Gerstein $\mathrm{H}$, et al. Relationship of metabolic syndrome and fibrinolytic dysfunction to cärdiovascular disease. Circulaton. Jul $292003 ; 108(4) 420-425$.

30. Balk EM, Lau J. Coudas LC, et al. Effects of statins on nonlipid serum markers atssociated with cardiovascalar disease: a systenatic review. Ann hntern Med. Oct 21 2003; 139(8):670-682.

31. Sakabe $\mathrm{K}$. Fukuda $N$, Wakayama $K$. et al. Lipid-altering changes and pleiotropic effects of atorvastatin in parkens with hyperchollesterolenina. Am I Cardiol. A tug 15 2004;94(4);497-500.

32. Wolfrum S, Jensen KS, Liao JK. Endohelium-dependent effects of statins, Arterioscler Thromb Vaw Biol. May 1 2003;23(5):729-736.

33. Georgieva AM, Cate HT, Keulen ET, et al. Prothrombotic markers in familial combined hyperlipidemiat evidence of endothelial cell activation and relation to metalbolic syndrome. Atherosclerosis. Aug 2004:175(2):345-351.

34. Ross SD. Allen IE, Connelly JE, et al. Clínical outcomes in statin treatment trials: a meta-analysis. Arch Invern Med. Aug 9-23 1999;159(15):1793-1802.

35. Yki-Jaryissen H. Thiazolidinediones. N Engl J Med. Sep 9 2004:351(11):1106-1118.

36. Riches FM, Watts GF, Hut J, et al. Reduction in visceral adipose tissue is associated with improvement in apolipoprotein B-100 metabolism in obese men. J Clin Entacrinol Metab. Aug 1999;84(8):2854 2861. 
Chapter 7

\section{General Discussion}




\section{Contents}

1. Dyslipidemia as a consequence of ectopic fat accumulation

2. Possible mechanism underlying VLDL-overproduction in FCHL; hepatic substrate availability

3. VLDL heterogeneiry can explain the phenotypes in FCHL

4. FCHL and metabolic syndrome

5. Hepatic lipid metabolism in FCHL - an open field for research

6. Activated coagulation profile can be an important cardiovascular risk factor in FCHL

7. Lipoprotein subclasses as a diagnostic approach in FCHL

8. Lipid-lowering therapy in FCHL

9. Conclusions 
The aim of this thesis was to study the phenotypic heterogeneity in $\mathrm{FCHL}$ with regard to lipoprotein subclasses. We assessed the role of VLDL-TG in the expression of the FCHL phenotype, and hypertriglyceridemia in particular. A numerical expansion of plasma VLDLI lipoproteins can explain, for a large part, major abnormalities in lipid and lipoprotein profile in FCHL subjects, such as hypertriglyceridemia (chapter 5), sd LDL, low HDL (chapter 2), and potentially relate to perturbations in blood coagulation profile such as elevated $\mathrm{PAI}-\mathbb{1}$ levels (chapter 4). The cross-sectional design of our studies prevents us from a definite conclusion on the exact pathogenic mechanisms operating in FCHL. It provides us, however, with enough material, which in combination with evidence in literature, allows us to draw a hypothesis. We believe that alterations in liver lipid metabolism mediate the expression of FCHL, especially in the setting of insulin resistance and hepatic lipid accumulation, both of which are very common in FCHL cases.

Here we will discuss our findings on the metabolic disturbances in FCHL in more detail.

\section{Dyslipidemia as a Consequence of Ectopic Fat Accumulation}

The complex FCHL phenotype results from the interaction of several genes, gene products and the environment ${ }^{1}$. Although their genetic nature is not completely understood, two primary pathogenic mechanisms operate in FCHL: 1) compromised fatty acid handling in response to insulin in adipose tissue, and 2) hepatic overproduction of Apo B 100 -containing lipoprotein particles, mostly as VLDL-Apo B.

Current insight suggests a basic defect in adipose tissue lipid metabolism in FCHL subjects. This defect manifests itself as increased fatty acid fluxes mainly in the postprandial state, and may represent an impaired fatty acid storage as triglycericles in response to insulin ${ }^{2}$. ". Increased FFA fluxes can be redistributed to other organs, and are most likely responsible for the ectopic fat accumulation observed in subjects with FCHL; their abdominal obesity ${ }^{4}$, accumulation of fat in liver (chapter 3), and potentially in muscle. Clinical observations suggest that expression of FCHL is sensitive to fat mass in the body (obesity) ${ }^{5}$, which in turn is responsible for greater fatty acid fluxes ${ }^{6}$. Of note, ectopic accumulation of fatty acids and fatty acid-derived metabolites can result in acquired organ insulin resistance? 
The adverse effects of ectopic fat accumulation on metabolism, including increased insulin resistance and dyslipidemia have become apparent from clinical practice. Conditions associated with disturbances in TG storage, such as lipodystrophies, are characterized by accumulation of fat in the visceral compartment, liver and skeletal muscles ${ }^{8}$. Adipose tissue dysfunction is a knowi side effect of highly active antiretroviral therapy of HIV infected patients. Interestingly, these patients show an abnormal metabolic profile, quite similar to that exhibited by hypertriglyceridemic FCHL patients".

Ectopic fat accumulation appears to mediate the expression of $\mathrm{FCHL}^{4,10,11}$. Our laboratory has reported on a strong relationship between abdominal obesity and hypertriglyceridemia in $\mathrm{FCHL}^{4}$. However, FCHL subjects show higher plasma Apo B, triglycerides and insullin levels for any degree of abdominal obesity compared to controls ${ }^{12}$, suggesting the additional contribution of genetic factors. For instance, the propensity of liver to produce an excess of VLDL particles can relate to genetic predisposition to lipogenesis de novo, mediated by USF-1 ${ }^{13}$. USF-1 is a transcription factor known to activate fatty acid synthesis in response to increased plasma glucose ${ }^{13}$. Noteworthy, an increased flux of glucose to the liver in FCHL patients is plausible, because of insulin resistant glucose utilization in the skeletal muscle of these patients ${ }^{14}$. The USF-1 gene has been recently linked to FCHL, but strong biological evidence that implicates it as an aetiogical factor in FCHL is still missing ${ }^{15}$. Yet, enhanced lipogenesis de novo can be an important additional factor in hepatic fat accumulation in FCHL, especially under conditions of increased stubstrate flux. We were the first to report on the high prevalence of fatty liver in $\mathrm{FCHL}$ : it was present in $76 \%$ of the FCHL probands that we studied, specifically the hypertriglyceridemic subjects ${ }^{16}$ (chapter 3 ). It is of interest that hepatic lipoprotein production is regulated, anong others, by insulin and the intracellular lipid content. These observations combined suggest a potentiat relationship between steatosis and hepatic lipoprotein overproduction in FCHL, manifested as hypertriglyceridemia.

In summary, increased flux of FFA alone, or in a combination with a genetic propensity to lipogenesis de novo, can challenge the storage capacity of liver, compromise intracellular insulin signalling ${ }^{7}$, and result in enhanced VLDL production in FCHL. 


\section{Possible Mechanism Underlying VLDL-Overproduction in FCHL; Hepatic Substrate Availability}

FCHL subjects exhibit overproduction of VLDL-Apo $\mathrm{B}^{\text {I7 }}$. There is a discussion in literature as to what drives the VLDL overproduction: altered substrate flux (discussed in the previous paragraph) or excess of cholesterol, phospholipids or triglyceride in the hepatocyte ${ }^{18}$. For example, there is a strong correlation between markers of activated cholesterol synthesis and VLDL-Apo $B$ production rate in man ${ }^{19}$. In vitro, the rate of $A$ po-B secretion of $H$ HepG2 cells is correlated with the intracellular mass of cholesterol esters, and not with the intracellular triglyceride mass ${ }^{20}$. Hyperinsulinemia stimulates cholesterol synthesis in the hepatocyte via enhanced SREBP activity ${ }^{21}$. Thus, an activated cholesterol synthesis pathway in the liver in the setting of hyperinsulinemia, can potentially account for hypersecretion and the presence of cholesterol-enriched atherogenic VLDL particles in $\mathrm{FCHL}^{22-24}$ (chapter 5).

Other investigators argue that Apo B-lipoprotein secretion is regulated by the rate of the triglyceride biosynthesis and not by the intracellular cholesteryl ester pools, at least in hepatic cell cultures ${ }^{25}$. Availability of phospholipids, particularly phosphatidylcholine, seems to be essential for VL.DL assembly as well ${ }^{26}$. Phospholipids comprise the surface of lipoproteins, and can be important in the initiation of VLDL synthesis by formation of Apo Bphospholipids complexes. Besides, phosphatidylcholine can be hydrolyzed through the activity of phospholipase D (PLD) to diacylglycerol, which in turn can be esterified to triacylglycerol for core lipid incorporation in VLDL, or serve as a precursor of phospholipid synthesis ${ }^{27}$. Accordingly, mice with phospatidylcholine deficiency in liver have reduced plasma VLDL-Apo B and VLDL-TG concentration ${ }^{28}$.

Taken together. VLDL assembly and secretion is a complex process, subjected to intricate regulation. Elucidating the pathways involved in VLDL production can help identify new target enzymes for effective lipid-lowering drug therapy. 


\section{VLDL Heterogeneity Can Explain the Phenotypes in FCHL}

FCHL subjects exhibit elevated plasma triglycerides (VLDL) and/or cholesterol (LDL), giving rise to three separate lipoprotein phenotypes (Fredrickson IIa, IIb, IV). Family members can show different phenotypes and some subjects can change from phenotype over time $^{23}$. This multiple lipoprotein phenotype is considered intrinsic to FCHL, and still puzzles investigators. To our view, the ability of the liver to produce a population of heterogeneous Apo-B 100 containing lipoproteins provides a plausible biologic explanation for this phenomenon in FCHL (discussed in chapter 2). Apo-B 100 containing lipoproteins produced by the liver are predominantly VLDL lipoproteins, but the density can range from LDL to large VLDL particles (VLDL1). Stable isotope studies have shown overproduction of Apo B 100 as VLDL as well as LDL lipoproteins in FCHL ${ }^{17.29}$. It is possible that the type of Apo-B 100 containing lipoproteins, produced by the liver of a particular FCHL subject is largely determined by the hepatic triglyceride availability for incorporation in lipoprotein particles ${ }^{29}$. (Figure 1). Hepatic triglyceride availability, in turn, is a function of genetic variations that affect hepatic fatty acid handling and storage, as well as fluctuations in FFA flux in response to environment. Of note, we found an increased liver TG content, as measured with ultrasound, in the majority of the FCHL probands we studied, in particular the hypertriglyceridemic ones (chapter 3).

Another factor that seems to play an important role in the process of lipoprotein production is hepatic sensitivity to the regulatory role of insulin. Insulin regulates the process of VLDL synthesis and secretion by several mechanisms (see Figure 1 and introduction). Moreover, insulin has a separate regulatory effect on the individual VLDL subclasses. Insulin inhibits hepatic VLDL1 production in healthy subjects, but not VLDL2 production ${ }^{30-32}$. Insufficient suppressive action of insullin on VLDL1 production contributes to increased lipid levels in type $2 \mathrm{DM}^{33}$. Expansion of plasna VLDL1 lipoproteins in FCHL occurs in the face of increased plasma insulin (chapter $\mathbf{2}$ and $\mathbf{5}$ ). Therefore, it is possible that alterations in hepatic lipid metabolism, involving an impaired response to insulin can contribute to excess VLDL1 production at least in some FCHL subjects. 


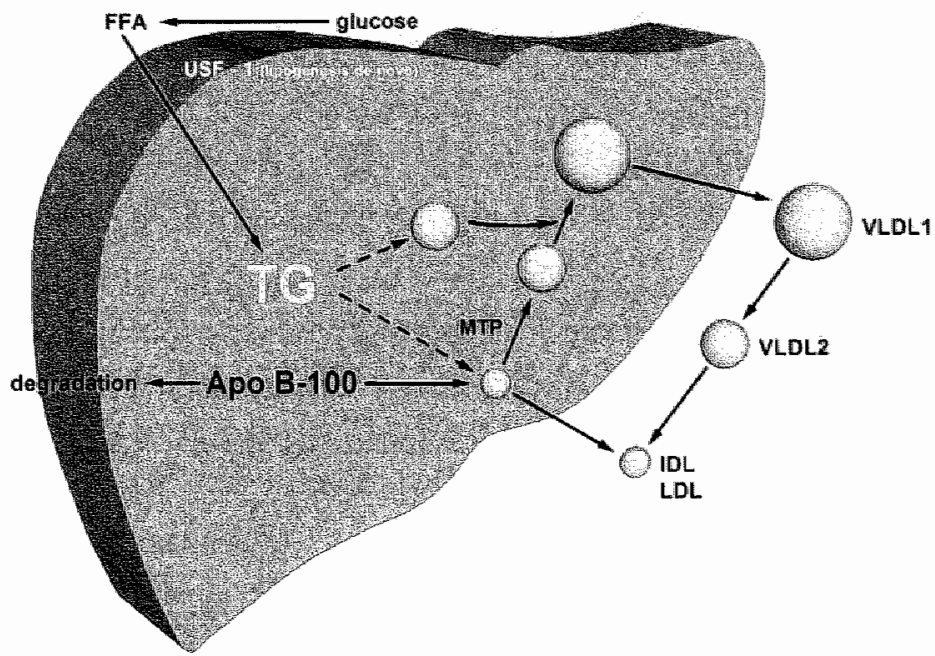

Figure 1. Hepatic production of heterogeneous Apo-B containing lipoproteins can underlie the multiple lipoprotein phenotype in FCHL.

Hepatic synthesis of lipoproteins particles features lipidation of Apo $\mathrm{B} 100$ protein in several steps: 1) Formation of pre-VLDL particle, catalyzed by microsomal transfer protein, MTP; 2) Addition of Apo B-free lipid mass to the pre-VLDL particle; a VLDL2 or VLDL1 particle can be formed depending on the amount of neutral lipid. For example, VLDL I contains 2 to 4-fold times more triglyceride molecules per particle than VLDL2 (15-20000 triglyceride molecules in VLDLI vs. 5-10000 in VLDL2). Whether a VLDL1 or a VLDL2, or in some cases IDL or LDL particle is formed, is dependent, among others, on the inhibitory regulation of insulin; the amount of fat in the liwer; and the substrate flux (glucose, FFA). These fluxes and the size of TG pool can be increased in FCHL.

Legend: dashed line signifies pathways inhibited by insulin 
Importantly, VLDL subclasses appear to have different catabolic fate in plasma: Excess of VLDL1 can induce fomation of sd LDL, and are mostly removed as VLDL remnants, whereas VLDL2 are preferentially catabolized to large, buoyant LDL ${ }^{34}{ }^{35}$ (Figure 2). Reciprocal relationship between VLDL and LDL cholesterol was observed in earlier studies on FCHL, suggesting that these lipoprotein classes can be linked through a reciprocal regulation ${ }^{23}$. We extended these observations with VLDL and LDL subclass analysis. The data showed that FCHL subjects who had different VLDL subclasses, differed in plasma triglycerides, $\mathrm{LDL}$ size, Apo $\mathrm{B}$ concentration and insulin as well, thus giving rise to aggregate metabolic phenotypes (chapter $\mathbf{2}$ and $\mathbf{5}$; the hypertriglyceridemic phenotype is summarized in Figure 2). Altogether, we suggest that alterations in hepatic Apo-B lipoprotein metabolism in terms of VLDL1 or VLDL2 production offer a coherent metabolic explanation of the multiple lipoprotein phenotype in FCHL.

NORMOLIPIDEMIC CONTROLS

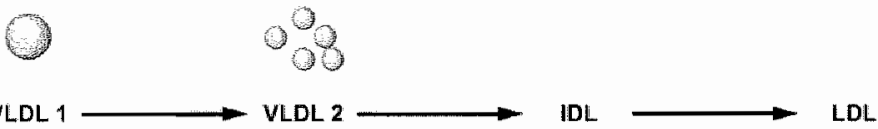

HYPERTRIGLYCERIDEMIC FCHL
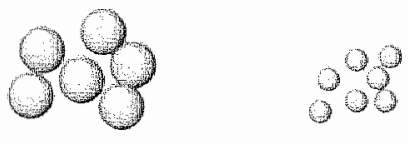

VLOL 1

VLLL 2

IDL sd LDL

Figure 2. Alteration in the VLDL-TG pathway in hypertriglyceridemic FCHL subjects Accumulation of triglyceride-rich particles VLDL $l$ in plasma restllts in the formation of sd LDL particles in FCHL, because of the existing metabolic channeling between VLDL and LDL subclasses. 


\section{FCHL and Metabolic Syndrome}

FCHL subjects exhibit a complex insulin resistant phenotype with a high prevalence of the metabolic syndrome components such as abdominal obesity, hypertriglyceridemia and hypertension (ii.e. dyslipidemic hypertension in FCHL) ${ }^{4111,36}$. A recent study identified the presence of the metabolic syndrome, as defined by National Cholesterol Education Program Adult treatment III panel (ATP III), in the majority of FCHL patients (65\%) compared to $19 \%$ in controls ${ }^{37}$. Our studies showed similar results (chapter 5).

As discussed in the previous paragraph, overproduction of VLDL against a background of insulin resistance can explain hypertriglyceridemia, and the associated changes in HDL and LDL subclasses in FCILL. In addition, VLDL overproduction most likely contributes to the considerable phenotypic overlap between FCHL and the metabolic syndrome (discussed in chapter 5). Propensity to overproduction can relate to a genetic polymorphism on chromosome $1 \mathrm{q}^{15}$. Pajukanta et al. recently proposed USF-1, located on $1 \mathrm{q} 23$ - 31 as a candidate gene in hypertriglyceridemic FCHL men ${ }^{13},{ }^{3}$, Polymorphisms in USF-1 gene can contribute to metabolic alterations and atherosclerosis, because of its regulatory role in lipogenesis, adipose tissue metabolism, systemic blood pressure, and $\mathrm{HDL}$ metabolism $^{15}$. Interestingly, metabolic syndrome was linked to the same genetic locus in an independent study, the Insulin Resistance Atherosclerosis Family Study ${ }^{39}$. Thus, USF-1 activity can mediate, at least in part, expression of the metabolic syndrome in FCHL ${ }^{13}$.

It is of interest that patients with type $2 \mathrm{DM}$, another disorder with high prevalence of the metabolic syndrome, exhibit hepatic production of much larger, lipid enriched VLDLI particles than controls, at least according to some studies ${ }^{40}$. By contrast, WLDL1 particles in FCHL subjects are of normal size (chapter 5). Therefore, it is likely that biochemical and genetic differences in hepatic lipid metabolism exist between FCHL and other forms of the metabolic syndrome or at least subjects with type 2 DM.

FCHL was identified 3 decades ago as genetic disorder of lipid metabolism. Although we have advanced our knowledge on lipid metabolism considerably, the focus on lipid metabolism alone could not help us unravel the genetic nature of FCHL. Numerous studies on FCHL have indicated that the metabolic background of FCHL is much more complex. We believe that the lipid and lipoprotein profiles in FCHL are the consequences of the underlying 
genetic and metabolic disturbances such as impaired adipose tissue function, insulin resistance and abdominal obesity. An expansion of plasma VLDL1 lipoproteins characterizes the metabolic syndrome in FCHL, and develops against a background of insulin resistance and visceral obesity. We propose that FCHL can be regarded as a genetic form of the metabolic syndrome, characterized by a propensity to overproduction of VLDL-Apo B particles.

\section{Hepatic Lipid Metabolism in FCHL - an Open Field for Research}

Hepatic lipid metabolism has not received much attention in FCHL, and one reason is that it is extremely difficult to study. It is difficult to obtain human hepatic tissue for studies, mostly out of ethical reasons. Moreover, a proper animal model for FCHL does not exist, and hepatic culture ceills from FCHL subjects are not available. An interesting approach in this regard, are the experiments of van Greevenbroek et al., who cultivated Hep2 cells with hyperlipidemic plasma of FCHL subjects, and observed a different protein response in comparison to cells cultured with control plasma ${ }^{41}$. An alternative to in vitro studies can be to evaluate substrate fluxes and VLDL production with stable isotopes, especially in the framework of an interventional study with a therapy known to improve adipocyte function and insulin resistance, such as thiazolidinediones (TZD) or life-style changes ${ }^{42}$. Moreover, genes involved in VLDL-TG pathway (Appendix) can be included as candidate genes in linkage and association studies.

Novel mechanisms of hepatic insulin resistance have been proposed recently. Changes in the redox state in the hepatocyte, related to oxidative stress can induce insulin resistance and affect VLDL production ${ }^{43}$. Endoplasmatic reticulum stress has been implicated in obesity and type $2 \mathrm{DM}$ as a mediator of insulin resistance ${ }^{44}$. The potential role of disturbed endloplasmatic reticulum function in hepatic insulin resistance in FCHL remains to be evaluated. 


\section{Activated Coagulation Profile Can Be an Important Cardiovascullar Risk Factor in FCHL}

Hyperlipidemic FCHL subjects exhibited a prothrombotic phenotype (chapter 4). This observation can be interpreted as an activation of the vascular endothelium in response to plaque burden and can relate to the observed susceptibility of premature acute coronary events in FCHL. Moreover, increased PAI-1 activity was specifically observed in hypertriglyceridemic FCHL subjects with features of the metabolic syndrome. An increase in PAl-1 activity indicates impaired fibrinolysis and may provide an additional cardiovascular risk factor in these subjects.

Several mechanisms can explain the increase in PAI-1 activity. Endothelial dysfunction due to the metabolic stress, related to hyperinsulinemia, hypertriglyceridemia or elevated plasma FFA, can lead to increase in PAI-1 levels ${ }^{45}, 46$. Also adipocytes can be an important factor in the production of PAI- 1 in insulin resistant conditions ${ }^{47}$. A part of our study "Lipitor, Inflammation and Gene Expression in Subcutaneous Adipose Tissue in FCHL (LIGEAT)" has been designed to address this issue (chapter 6). A novel mechanism that can contribute to plasma PAI-1 levels in FCHL subjects is the presence of fatty liver ${ }^{9}$. The high prevalence of nonalcoholic fatty liver disease in hypertriglyceridemic FCHL subjects ${ }^{16}$ is a potential explanation for the elevated PAI-1 activity. Interestingly, PAI-1 gene activity can be regulated by USF-1 ${ }^{49}$, which has been implicated in FCHL subjects with elevated plasma triglycerides ${ }^{13}$. Therefore, PAI 1 is a potential marker of USF 1 activity in FCHL, and elevated activity of PAI-1 in hypertriglyceridemic FCHL subjects can be particularly inherent to the nature of FCHL.

\section{Lipoprotein Subclasses as a Diagnostic Approach in FCHL}

Preponderance of Sd LDL is an inherent feature of FCHL. It has been proposed as a diagnostic marker of the disorder ${ }^{50}$, because it captures both pathogenic mechanisms and the increased risk of premature CAD in FCHL. We examined the sd LDL phenomenon in FCHL (chapter 2). We found a close association between sd LDL and the accumulation of 
triglyceride-rich VLDL particles, or VLDL1, which characterize the hypertriglyceridemic phenotype in FCHL. Based on this observation and sound evidence from literature, we conclude that in FCHL subjects (who have a background of insulin resistance and propensity to overproduction) hepatic VLDL1 particles are the precursor of sd LDL in plasma.

Sd LDL is a final step in a pathway; therefore all plasma lipid modifier genes contribute to its expression. Thus, presence of sd LDL reffects predominance of VLDL 1 particles as well as prolonged circulation times, CETP and HL activities, LDL receptor defects, etc. However, sd LDL is predominantly assaciated with the hypertriglyceridemic FCHL phenotype (chapter 2). Is expansion of plasmaVLDL1 lipoproteins a better marker of FCHL, than the presence of sd LDL? More studies are needed to determine how specific this feature is to FCHL. Comparison to other disorders with overproduction with regard to lipid and apolipoprotein composition (e.g. Apo CIII, Apo E) will be required.

The main endpoint in diagnosis and treatment of dyslipidemia is prevention of acute coronary events. Prospective studies have demonstrated that presence of $s d \mathrm{LDL}$ is associated with increased cardiovascular risk, independent of plasma lipid levels ${ }^{51}$. Yet, another study in men with cardiovascular disease showed that those with large VLDL particles were 3 to 4 times more likely to have extensive CAD than those without, independent of plasma triglyceride and $\mathrm{HDL}$ cholesterol ${ }^{52}$. By contrast, adjustment for these variables greatly reduced the relation of $s d \mathrm{LDL}$ to $\mathrm{CAD}^{52}$. Taken together, an implication of these and our studies is that detailed analyses of lipoprotein subclasses can advance our knowledge on dyslipidemia, and potentially help identify those patients with increased risk for acute coronary events. However, analytical ultracentrifugation, which we used for VLDL subclasses analysis in this thesis, is laborious and difficult to use on a large scale, or for clinical purposes. Therefore, development of new methods for faster determination of lipoprotein subclasses can offer new insights and adwance our capability of diagnosing dyslipidemias and cardiovascular disease ${ }^{52}$. 


\section{Lipid-Lowering Therapy in FCHL}

Treatment with statins (atorvastatin, $40 \mathrm{mg}$ daily for 8 weeks) effectively lowered plasma lipiid levels in FCHL (chapter 6). Increased hepatic uptake of Apo B-containing particles is most likely the mechanism responsible for lowering of plasma Apo-B containing lipoproteins ${ }^{53}$. The observed improvement in lipid profile underscores the contribution of a relative clearance defect of Apo B-containing lipoproteins to hyperlipidemia, especially hypercholesterolemia in FCHL.

FCHL often manifests itself with a combination of elevated plasma triglycerides, low HDL cholesterol, and predominance of sd LDL, a lipoprotein phenotype considered to be particularly atherogenic ${ }^{54}$. We found that statin treatment ameliorated but failed to resolve the atherogenic lipoprotein phenotype in most FCHL subjects, in consistence with previous reports in literature ${ }^{55}$. One explanation can be that statins do not resolve overproduction, because this medication cannot counteract insulin resistance, and insulin resistance is most probably the cause of excessive VLDL1 production ${ }^{56}$. Additional factor that can contribute to the persistence of sd LDL and low HDL cholesterol in FCHL subjects is the activity of HL, which is known to be increased in insulin tesistance ${ }^{32,54}$.

Fibrates (PPAR- $\alpha$ agonists) are more efficient than statins in reducing elevated triglyceride levels and increasing $\mathrm{HDL}$ cholesterol. A small, but significant increase in LDL. size have been observed with fibrate monotherapy in FCHL subjects ${ }^{55}$. However, the same study showed that fibrate monotherapy hats little effect on total plasma LDL cholesterol in $\mathrm{FCHL}^{55}$. Therefore, a combination of a fibrate with a statin can be the optimail therapy for FCHL patients, especially those with combined hyperlipidemia. In addition, fibrates affect hepatic fatty acid oxidation through activation of PPAR- $\alpha$, and thus can affect hepatic fat content and poientially improve fatty liver ${ }^{57}$. Fibrate therapy, however, can recuire extra caution, especially in elderly FCHL patients, because of reported increased risk of Thabdomyolysis. It is of interest that large-scale trials designed to evaluate if fibrate treatment is effective with regard to CAD prevention are being conducted at the moment ${ }^{\text {5i⿱ }}$. The results of these studies are eagerly awaited.

Ectopic fat accumulation and insulin resistance can precede or deteriorate the lipoprotein abnormalities observed in subjects with FCHL (discussed in chapter 5). Therefore, 
therapies that aim at ameliorating visceral obesity, fatty liver or insulin resistance are potentially beneficial in FCHL, such as the PPAR-y agonists, TZD, or life style changes ${ }^{59}$. TZD are a new class of drugs, with an insulin sensitizing action ${ }^{42}$. Treatment with TZD promotes fat mobilization from the liver or the visceral area and its redistribution to the subcutaneous fat depot ${ }^{42}$. However, the effect of TZD on the plasma lipoprotein profile in FCHL has not been addressed yet. It still remains to be established if TZD lower the risk of CAD.

In summary, statin therapy is important for prevention of acute events in FCHL patients, due to its pleiotropic effects, such as lipid-lowering, anti-proinflammatory and antiprothrombotic action. The atheroprotective effects of statin therapy have been demonstrated in large prospective studies ${ }^{60}$. However, hypertriglyceridemic FCHL patients might have an additional benefit from therapies that target insulin resistance.

\section{Conclusions}

In conclusion, there is strong evidence that suggests that FCHL should be regarded as a genetic form of the metabolic syndrome, associated with a propensity to overproduction Apo B-100 lipoproteins. Moreover, we argue that alterations in hepatic Apo-B lipoprotein metabolism in terms of VLDL1 or VLDL2 production offer a coherent metabolic explanation of the metabolic phenotypes in FCHL, in combination with altered lipoprotein elimination. The VLDL-TG pathway is central to the plasma lipid and lipoprotein phenotypes observed in FCHL. Recognition of the VLDL-TG pathway in FCHL is important with respect to emerging drug therapies, which target lipid metabolic enzymes, and can potentially manage the increased CAD risk, associated with the disorder. 


\section{References}

1. Eurlings PM, van der Kallen $\mathrm{CJ}$, Geurts IM, et ât. Genetic dissection of familial combined. hyperlipidemia. Mol Genet Metab. Sep-Oct 2001;74(1-2):98-104.

2. Aitman TJ, Godsland IF, Farren B, at al. Defects of insulin action on farty acid and carbohydrate metabolism in familial combined hyperlipidemia. Atterioscler Thromb Vasc Biol. Apr 1997":17(4):748754.

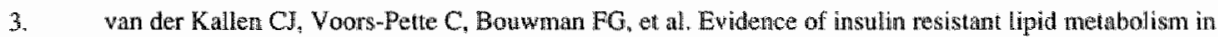
adipose tissue in fomilial combined hyperlipidenaia, but not type 2 diabetes mellitus. Atherosclerosis. Oet $2002 ; 164(2): 337-346$.

4. van der Kallen CJ, Woors-Pette C, de Bnuin TW. Abdominal obesity and expression of familial conbined hyperlipidemia. Obes Res. Dec 2004; 12(12):2054-2061.

5. Ascrso JF, Sales J, Merchante A, at anfluence of obesily on plasna lipoproteins, glycaemait and insulinamia in patients with fanillal combined hyperlipidaemu. Int J Obes Relat Metab Disord. May $1997 ; 21(5): 360-366$.

6. Castro Cabezas M, de Bruin TW, de Valk HW, et al. Impaired fatty acid metabolism in familial combined hyperlipidemia. A mechanism associating hepatic apolipoprotein $\mathbf{B}$ overproduction and insulin resistance. J Clin Invest. Jul 1993;92(1):160-168.

7. Satiel AR, Kahn CR. Insulin signalling and the regulation of glucose and lipid metabolism. Nature. Dec $132001 ; 414(6865): 799-806$.

8. Garg A. Acquired and inher:ted lipodystrophies. N Engl I Med. Mar 18 2004;350(12):1220-1234.

9. Yki-Jarvinen $H$, Sutinen J, Silveira A, et al, Regullation of Plasma PAI-1 Concentrations in FAART. Associated Lipodystrophy During Rosiglitazone Therapy. Auterioscler Thromb Vasc Biol. Apr 1 $2003,23(4): 688-694$

10. Carr MC, Brumzell JD. Abdominal obesiry and dystipidemin in the metabolic syndrome: importance of type 2 diabetes and ramilial conbined hyperlipidemia in coronary artery disease risk. 1 Chin bindocrinol Metab. ILun 2004;89:6):2601-2607.

11. Keulen ET, Voors Pette C, de Bruin TW. Familial dyslipidemic hypertension syndrome: familial combined hyperlipidemia, and the role of abdominal fat mass. Am J Hypentersis Apr 2001; 1.40, Pt 1):357-363.

12. Purmel JQ, Kahn SE, Schwarz RS, et al. Relationship of insulin sensitivity and ApoB levels to intraabdominal fat in subjects with familial combirued hiyperlipidemat. Arterioscler Thromb Vasc Biol. Apr $2001 ; 21(4) ; 567-572$.

13. Pajukanta P, Lilja HE, Sinshemer IS, et al. Fambial combined hypenipidemia is associated with upstream transcription factor 1. (USF1). Nat Gene:. Apr 2004,36(4):371-376. 
14. Karjaiamen L, Piltajamaki J, Karhapal P, et al. Impaired insulin-stimulated glucose oxidation and free fatiy acidsuppression in patients shith lamilial combined hyperlipidemina a precursor defect for dysilipidemia? Arterioscler Thromb Visc Biol. Oet 1998;18(10):1548-1553.

15. Shouhders CC, Nroumova RP. USFl mplicated in the aetiology of familial combined hyperlipidaemiat and the metabolic syndrone. Trends Mol Med. Aug 2004;10(8):362-365.

16. de Bruin TW, Georgeva AM, Brouwers. MC, et all. Radiological evidence of nonalcoholic tatty liver disesse in familat combined hyperlipidemis. Am J Med. Jun 15 2004:116(12):847-849.

17. Venkatesan $S$, Cullen P, Pacy $P$, et all. Stable isotopes show a direct relation botween VLDL apoB overproduction ard serum triglyceride levels and indicate a metabolically and biochemically coherent bysis for familial combined hyperlipidemia. Arterioscler Thromb. Jul 1993;13(7);1110 1118.

18. Thompson GR, Naoumowa RP, Watts GF. Role of cholesterol in regulating apolipoprote in $B$ secretion by the liver. J Lipid Res. Mar" 1996;37(3):439-447.

19. Riches FM, Wats GF, Nacumova RP, et al. Direct association between the hepatic secretion of wery low-density lipoprotein apolipoprotein B-100 and plasna mevalonic acid and lathosterol concentrations in man. Altherosclerosis. Nov 1997;135(1):83-91.

20. Avramoglu $\mathrm{K}_{\mathrm{K}}$, Cicunflone $\mathrm{K}_{\mathrm{a}}$ Snidernam $\mathrm{AD}$. Role of the neutral lipid accessible pool th the regulation of secretion of apoBim 100 lipoprotein particles by HepG2 cells. I Lipid Res. Dec 1995:36(12):25132528.

21. Horton JD, Goldstein JL, Brown MS. SREBPs: activators of the complete program of cholesterol and fatty actid symthesis in the liver. J Clin Invest. May 2002; 109(9):1125-1131.

22. Wan Batlingen HH, Kock LA, de Man HH, et al. In vitro lipolysis of humar VLDL: effect of different VLDL compositions un normolipidemia, familial combined hyperlipidemia and familial hypertriglyceridemia. Atherosclerosis. Mar 1996;121(1):75-84,

23. Brumzell JD, Albers JJ, Chait A, et al, Plasma lipoproteins in familial combined hyperlipidemia and monogenic familian thypertriglyceridemia. J Lipid Res. Feb 1983;24(2):147-155.

24. Cabezas $\mathrm{MC}$, de Brein $\mathrm{TW}$, Janken $\mathrm{H}$, el al. Impaured chylontcron memant clearance in fantial combined lyperlipidemia, Atrerioscler Thromb. Jun 1993;13(6):804-814.

25. Benoist F, Grand-Penct T. ApoB-100 secretion by $\mathrm{HepO2}$ cells is regulated by the rate of triglycaride

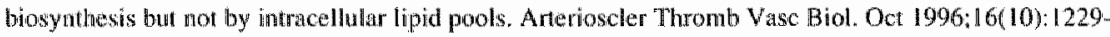
1235 .

26. Asp L, Claesson $C_{\text {. }}$ Boren J, et al. ADPmibosylation factor I and its activation of phospholipase D are inpontant for the assembly of very low density lipoproteins. J Biol Chem. Aug $252000 ; 275(34): 26285$ 26292.

27. Colenan RA, Lee DP. Enzynes of triacylglycarol synthesis and their regulation. Prog Lipid Res. Mar $2004: 432): 134-176$

28. Jacobs RI Devlin C., Tabas I, et al. Targeted deletion of hepatic CTP:phosphocholine cytidylylmansferase alpha in muce decreases plasma high density and very low density lipoproteins. I Biol Chem Nov $52004: 279(45): 47402-47410$. 
29. Kissebah AH, Alfarsi S, Evans DJ. Low density lipoprotein metabolism in familial combined hyperlipidemia. Mechanism of the multiple lipoprotein phenotypic expression. Arterioselerosis. NovDec 1984;46(6):614-624

30. Malmstrom R, Packard CJ, Caskeke M, et al. Effects of insulin and acipimox on WLDL 1 and VLDL2 apolipoprotein B production in normal subjects. Diabetes. May 1998;47(5):779-787.

31. Malmstrom R, Packard CJ, Watson TD, et al. Metabolic basns of hypotriglyceridenic effects of insulin in nomal men. Arterioscler Thromb Vase Biol. Jul 1997; 17(7);1454-1464.

32. Gill JM, Brown JC. Bedford D, et al. Hepatic production of VLDL.1, but not VLDL2 is related to insulin resistance in normoglycaemic middle-aged subjects. Atherosclerosis. Sep 2004:176(1):49\%:56.

33. Malmstrom $\mathbb{R}$, Packard $C J$, Caslake $M$, et al. Defective regulation of triglyceride netabolism by insulin in the liver in NIDDM. Diabetologia. Apr 1997:40(4):454-462.

34. Packard $\mathrm{CJ}$, Shepherd I. Lipoprotein heterogeneity and apolipoprotein B metabolism. Arterioseler Thromb Vasc Biol. Dec 1997;17(12);3542-3556.

35. Bemeis KK. Krauss RM. Metabolic origins and clinical significance of LDL heterogeneity. 3 Lipid Res. Sep 2002;43(9):1363-1379.

36. Voors-Pette C, de Bruin TW. Excess coronary heart disease in Fumilial Combined Hyperlipidemia, in relation to genetic factors and central obesity. Atherosclarosis Aug 2001;157(2);48 1-489.

37. Hopkins PN, Heiss G, Ellison RC, et al. Coronary Arlery Disease Risk in Familial Combined Hyperlipidemia and Familial Hypertriglyceridemia. A Case-Control Comprison From the Nationat Heart, Lung, and Blood Institute Family Heart Study. Circulation. Jul 72003.

38. Shoulders CC, Jones EL, Naumova RP. Genetics of familiat combined hypentipidenia and risk of coronary heart disease. Hurn Mol Genet. Apto 1 2004;13 Spec No 1 R 149-160.

39. Langefeld CD, Wagenknecht LE. Ronter $\mathbb{I}_{\text {s }}$ et al. Linkage of the metabolic syndrome $101 \mathrm{q} 23-\mathrm{q} 31$ in Hispanic families: the Insulin Resistance Atherosclerosis Study Fanily Study. Diabetes. Apr $2004: 53(4): 1170-1174$

40. McEneny J, OKante MJ, Moles KW, et al. Very low density lipoprotein subfrachons in Type ll diabetes mellitws atterations is composition and susceptibility lo oxidation. Diabetologia. Apr 2000,43(4):485. 493.

41. van Greevenbroek MM, Verneulen VM, de Brum TW. Familial combined hyperlipidemian pasma stimulates protein secretion by HepG2 cells: identffication of fibronedin in the differental secretion proteone. J Lipid Res. Nov 2002;43(11):1846*1854.

42. Yki-Jarvinen H. Thizzolidinedioties. N Engl J Med. Sep $92004-35$ )(11):1106-1118.

43. Pan M, Cederbawn Al, Zhang YL, et al. Lipid peroxidation and oxidanat stress regukte bepatic: apolipoprotein $B$ degradation and VLDL production. I Clin Invest. May 2004:113(9):1277-1287.

44. Oran $U, C$ Co Q, Yillmaz E et al. Endoplasmic reiculum stress links obesity, insulin aktion, and type 2 diabetes. Science. Oct 15 2004:3066 5695$), 457-461$.

45. Kohler HP, Grant PJ. Plasminogen activator inhibitor ype I and coronary artery disease. N Engl I Med. Jun $152000 ; 342(24): 1792 \% 1801$. 
46. Reawen 6 . Metabolic syndrome: pathophysiology and implications for management of cardiovascular disease. Circulation: Hal 16 2002;106(3):286-288.

47. Kointinen HA, Duserre E. Ebeling $P_{\text {n }}$ et al. Subcutaneous adipose tissue expression of plasminogen activator inhibitor-1 (PAI-1) in nondiabetic and Type 2 diabetic subjects. Diabetes Metab Res Rev. SepOct $2000 ; 16(5): 364-369$

48. Aless MC, Bastelica D, Mavri A, et al. Plasma PAI-1 Levels Are More Strongly Related to Liver Steatosis Than to Adipose Tissue Accumulation. Arterioscler Thromb Vase Biol. Jul I $2003: 23(7): 1262-1268$

49. Zietz B, Drobnik $W$, Henfarth $\mathrm{H}$, et al: Plasminogen activator inthibitor-1 promoter actiwity in adipocytes is not inflenced by the $40 / 5 \mathrm{G}$ promoter polymorphism and is regulated by a USF-1/2 binding site immediately preceding the polymorphic region. J Mol Endocrinol Feb 2004;32(1):155-163.

50. Veerkamp MI, de Graaf J, Bredie SJ, et al. Diagnosis of familial combined hyperlipidemia based on Ilpid phenotype expression in 32 families: results of a 5-year follow-up study. Arterioscler Thromb Vatso Biol. Feb $12002 ; 22(2): 274-282$.

51. Si-Pierre AC, Ruel IL, Cantin B, et al. Comparison of various electrophorelic characteristics of LDL. particles and their relationship to the risk of ischemic heart disease. Circulation. Now 6 $2001 ; 104(19): 2295-2299$.

52. Freedman DS, Otvos JD, Jeyarajah Ed, et al. Rellation of hipoprotein subclasses as measured by proton nuclear magnetic resonance spectroscopy to coronary artery disense. Arterioscler Thromb Vasc Biol. Jul $1998 ; 18(7): 1046 \% 1053$.

53. Aguilar-Salinas CA, Hugh P. Barrett $\mathbb{R}$, at al. A familial combined hyperlipidemic kindred with impared apolipoprotein B catabolism. Kiretics of apolipoprotein $\mathrm{B}$ during placebo and pravastatin therapy. Arterioscler Turomb Vasc Biol. Jan 1997;17(1):72-82.

54. Allaye H, Aouizerat BE, Cantor RM, et al. Families with familial combined hyperlipidemia and fatmilias entiched for coronary artery disease share genetic deterninants for the atherogenic lipoprote in phenotype. Am J Hum Genet. Aug 1998;63(2):577-585.

55. Brodie St, de Bruin TW Demacker PN, et al. Comparison of gemfibrozil versus simvastatin in Lamilial combined linyperlipidemia and effocts on apolipoprotein-B-containing lipoproteins, low-density lipoprotein subfraction profile, and low-density lipoprotein oxidizability. Am a Cardiol. Feb 15 $1995: 75(5): 348-353$

56. Wats GF ${ }^{2}$ Chan DC, Barrett PH, ef ath. Effect of a statin on hepatic apolipoprotein B-100 secretion and plasma campesterol levels in the metabolic syndrome. Int J Obes Relat Metab Disord. Jut $2003,27(7): 862-865$

57. Sli $Y$, Burin P. Lipid metabolic enzymes: emerging drug targets for the treatument of obesity. Nat Rev Drug Discov. Aug 2004:3(8):695-710.

58. The need for a large-sicale trial of fibrate thetapy in diabetes. the rationale and design of the fenofibrite Intervention and Event Lowering in Diabetes (FIELD) study. [ISRCTN64783481\%. Cardiovasc Diaberol. Dec $12004: 3(1) ; 9$ 
59. Riches FM, Watts GF, Hua J, et all Reduction in visceral adipose tissue is associated with improvement in apolipoprotein B-100 metabolism in obese men. J Cin Endocrinol Metab. Aug 1999;84(8):28542861 .

60. Ross SD, Allen IE, Connelly JE, et al. Clmical outcones in statin mentment trials: a metawanalysis. Arch Interm Med. Aug 9-23, 1999;159(15):1793/1802. 
Appendix

Genetic Factors Implicated in the VLDL-TG pathway 


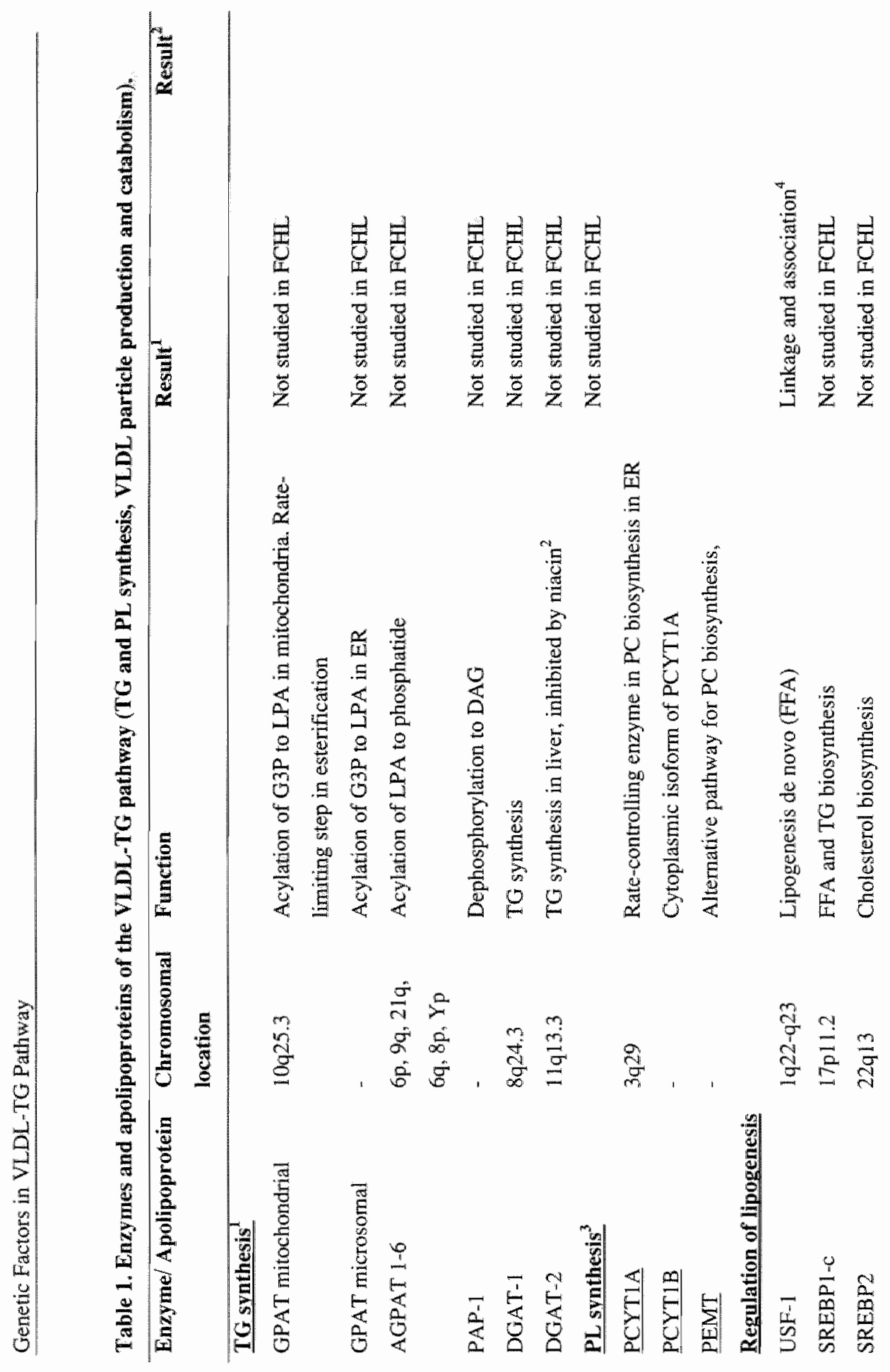




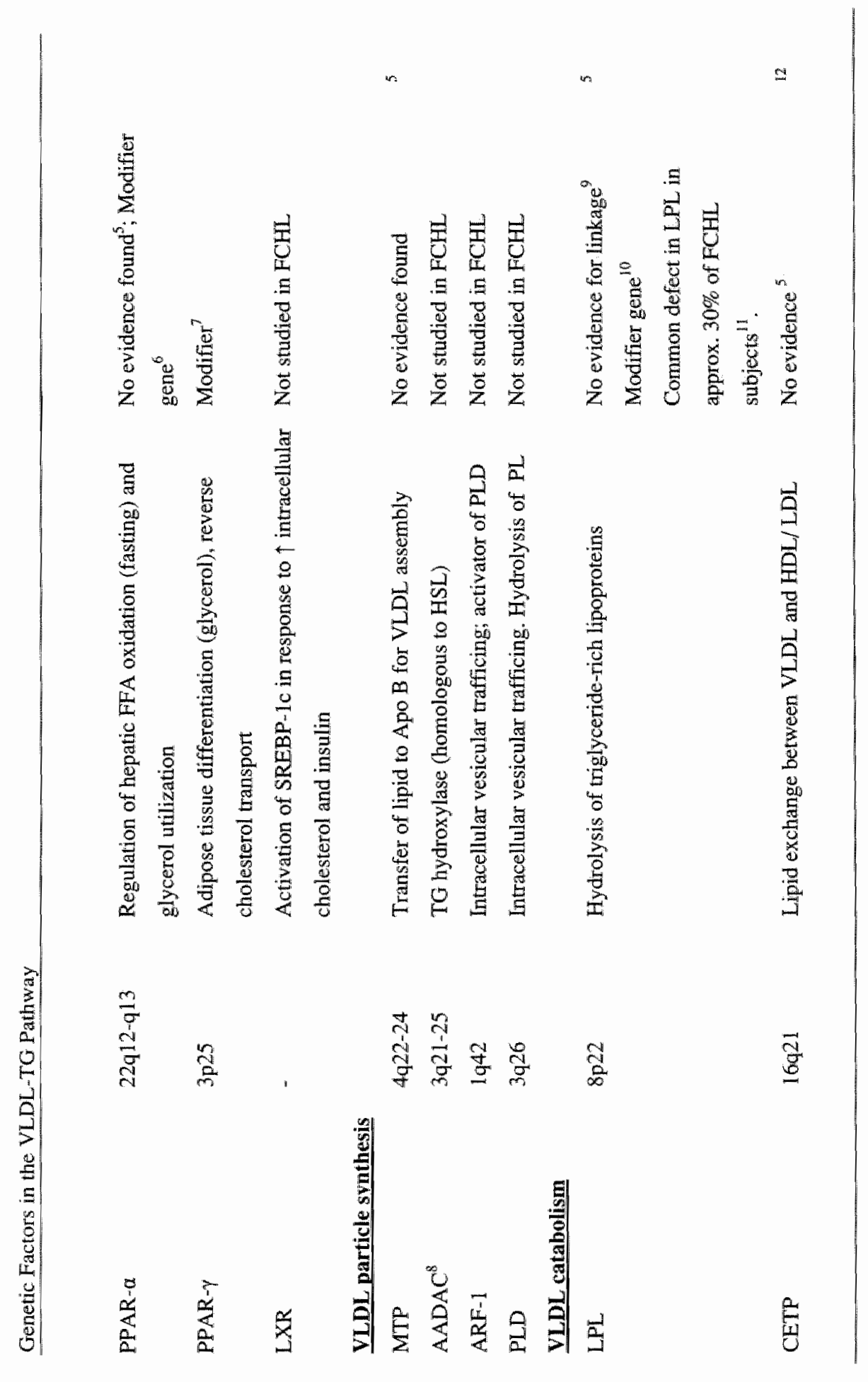




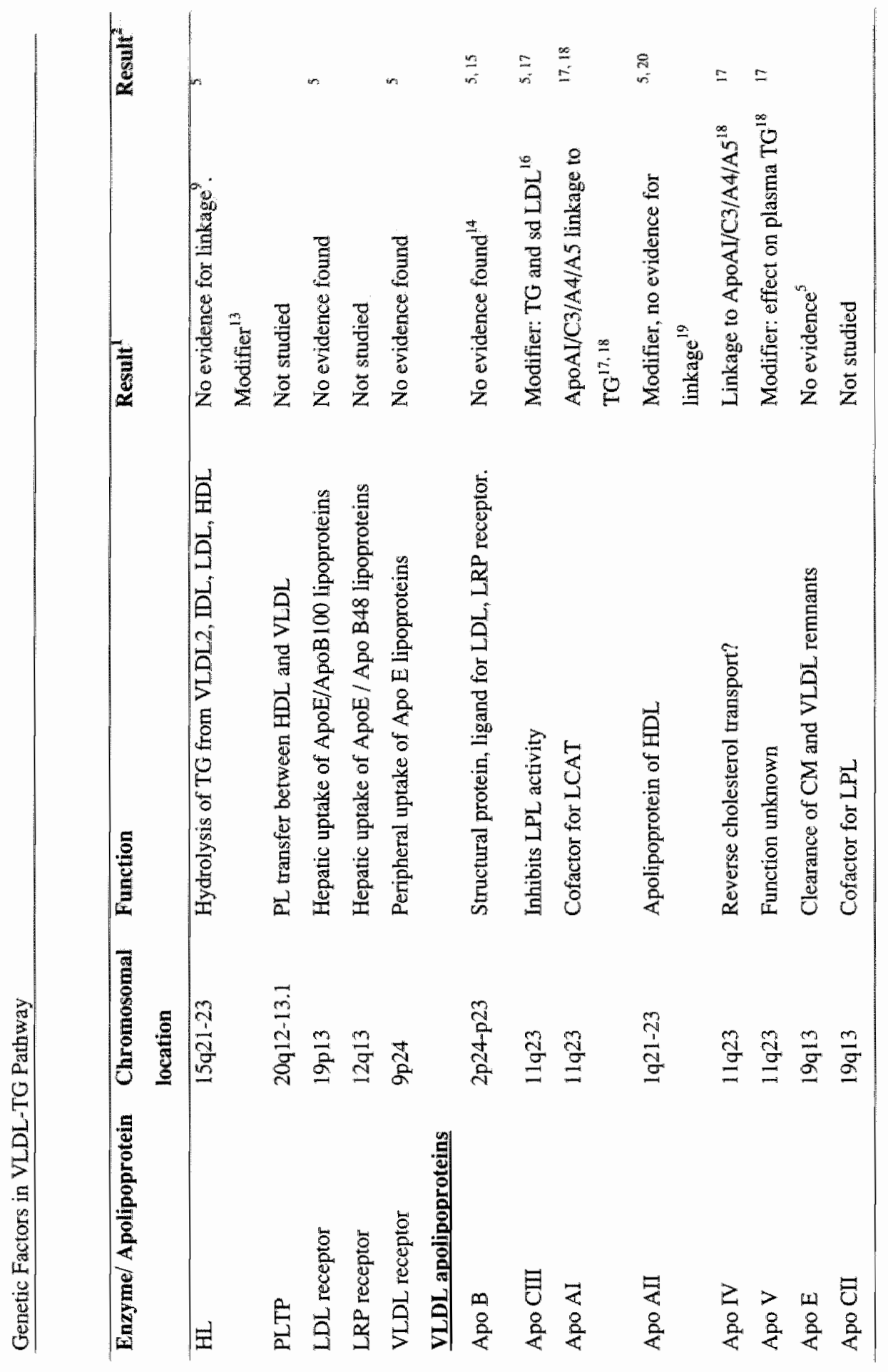




\begin{abstract}
Abbreviations:
GPAT $=$ Glycerol-3-phosphate acyltransferase; GSP $=$ Glycerol-3 phosphate; LPA $=$ Lysophosphatidic acid; ER = Endoplasmatic Reticulum; AGPAT = AGP acyltransferase/ Lysophosphatidic acid acyltransferase; $P A P=$ Phosphatidic acid phosphatase; DAG = Diacylglycerol; DGAT-1 = Diacylglycerol acyltransferase type 1;

DGAT-2 = Diacylglycerol acyltransferase type 2; PL = Phospholipid; PCYT 1A= Phosphate cytidylyltransterase $1 \mathrm{~A} ; \mathrm{PCYT} 1 \mathrm{~B}=$ Phosphate cytidylyltransferase $1 \mathrm{~B} ; \mathrm{PC}=$ Phosphatidylcholine; $\mathrm{PEMT}=$ Phosphatidylethanolamine $N$-methyltransferase: $\mathrm{AADAC}=$ Arylacetamide deacetylase : $\mathrm{ARF}-1=\mathrm{ADP}$ ribosylation factor 1; PLD = Phospholipase D

Result ${ }^{2}=$ study has been confirmed.
\end{abstract}

\title{
References
}

1. Coleman RA, Lee DP. Enzymes of triacylglycerol synthesis and their regulation. Prog Lipid Res. Mar $2004: 43(2): 134-176$.

2. Meyers CD, Kamanna VS, Kashyap ML. Niacin therapy in atherosclerosis. Curr Opin Lipidol. Dec 2004; 15(6):659-665.

3. Noga AA, Zhao Y, Vance DE. An unexpected requirement for phosphatidylethanolamine $N$ methyltransferase in the secretion of wery low density lipoproteins. J Biol Chem. Now I 2002;277(44):42358-42365.

4. Pajukanta P, Lilja HE, Sinsheimer JS, et all. Famillial combined hyperlipidemia is associated with upstream transcription factor 1 (USF1). Nar Genet. Apr 2004;36(4):371-376.

5. Aowizerat BE. Allayee $\mathrm{H}$, Cantor $\mathrm{RM}$, et al. Linkage of a candidate gene locus to familial conibined hyperlipidemia: lecithincholesterol acyltransferase on $16 \mathrm{q}$. Arferioscler Thromb Vasc: Biol. Nov $1999 ; 19(11): 2730-2736$.

6. Eurlings PM, van der Kallen CJ, Geurts $\mathrm{MM}$, et al. Identification of the PPARA locus on chromosiome $22 q 13.3$ as a modifier gene in familial combined hyperlipidernia. Mol Genet Metab. Dec 2002:77(4):274-281.

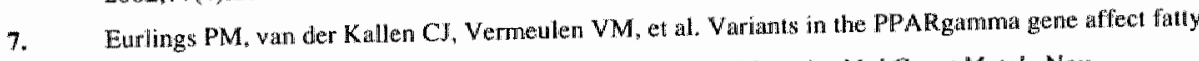
acid and glycerol metabolism in familial conbined hyperlipidemia. Mol Gener Metab. Nov $2003 ; 80(3): 296-301$.

8. Gibbons GF, Wiggins D, Brown AM, et al. Synthesis and function of hepalic very-low-density lipoprotein. Biochem Soct Trans. Feb 2004;32(Pt 1):59-64. 
4. Pajukanta P, Porkka $\mathrm{KV}$, Antikainen $\mathrm{M}$, et al. No evidence of linkatge between familial combined hyperlipudemia and genes encodling lipolytic enzymes in Finnish families. Arterioscler Throwb Vasc Biol. Mayy 1997:17(5):84:-850.

10. Campagna F, Montali $A_{a}$ Baroni MG, et al. Common variants in the lipoprotein lipase gene, but not those in the insulin receptor substratte-1, the beta 3 -adrenergic receptor, and the intestinal fatty acid bind ing protein-2 genes, influence the lipid phenotypic expression in familial combined hyperlipidemiat. Merabolism. Oct 2002;51(10):1298-1305.

11. Babirak SP, Brown BG, Bnuzell JD. Familiall combined hyperlipidemia and abnormal lipoprotei.n lipase. Arterioscler Thromb. Oct 1992;12(10):1176-1183.

12. Shoulders CC, Jones EL, Naoumowa RP. Genetics of familial combined hyperlipidemia and risk of coronary heart disease. Hum Mol Genet. Apr $12004 ; 13$ Spec No 1:R 149-160.

13. Hoffer MI, Snieder H, Bredie SJ, et al. The V73M mutation in the hepatic lipase gene is associated with elewatted cholesterol levels in four Dutch pedigrees with familial combined hyperlipidemia.

Atherosicterosis. Aug 2000;151(2):443-450.

14. Rauh $G$, Schuster H, Muller B, et al. Genetic evidence from 7 farnilies that the apolipoprotein $B$ gene is not involwed in familial combined hyperlipidemia. Acherosclerasis. Jul 1990;83(1):81-87.

15. Austin MA, Wijsman E, Guo SW, et al. Lack of ewidence for limkage between low-densitly lipoprotein subclass phenotypes and the apolipoprotein B locrus in familial combined hyperlipidemia. Cenet Eppidemiol. 1991;8(5):287-297.

16. Ribalta J, La Ville $\mathrm{AE}$, Vallve JC. et al. A wariation in the apolipoprotein C-III gene is associated with an increased number of circulating VLDL and IDL particles in familial combined hyperlipidemia. $J$ Lipid Res. Jun 1997;38(6):1061-1069.

17. Mar $R$, Pajukanta $P$, Allayee $H_{\text {n }}$ et al. Association of the APOLIPOPROTEIN Al/C3/A4/A5 gene cluster with triglyceride levels and LDL particle size in farallial combined hyperlipidemia. Cirtc Res. May 16 2004;94:(7):993-999.

118. Eichenbaum-Voline S, Oliwier $M_{n}$ Jones EL, et al. Linkage and association between distinct varians of the APOA1/C3/A4/As gene cluster and fannilial combined hyperlipidemia. Arterioscter Thromb Vasto Biol. Feb $2004 ; 24(1): 167-174$.

19. Pajukanta P. Nuotio I. Terwilliger JD, ei al. Linkage of familial combined hyperlipidaemia to chromosome 1q21-q23. Nat Genet, Apt 1998;18(4)×369-373.

20. Allayee H, Castellani LW, Cantor RM, et al. Biochenical and genetic association of plasma apolipoprotein A-II levels with farmilial combined hyperlipidemia. Circ Res. Jun $132003,92(11): 1262-$ 1267. 
Summary \& Samenvatting 


\section{Summary}

Familial Combined Hyperlipidemia (FCHL) is a genetic disorder of lipid metabolism, defined as a familial hyperlipidemia with a variable expression, i.e. isolated hypercholesterolemia (Fredrickson IIa), combined hyperlipidemia (IIb), or isolated hypertriglyceridemia (IV), in combination with increased familial prevalence of premature myocardial infarction. Affected FCHL subjects exhibit a complex metabolic phenotype, which can feature elevated plasma insulin concentrations in the presence of insulin resistance, abdominal obesity, and hypertension. A clustered entity of elevated plasma triglycerides, low high-density lipoprotein (HDL) cholesterol and predominance of small dense LDL. commonly described as the atherogenic lipoprotein phenotype, is frequently observed in FCHL subjects. We have studied this adverse phenotype and its metabolic determinanis in more detail.

There is evidence that the complex. FCHL phenotype results, at least in part, from: impaired fatty acid metaboiism, associated with impaired fatty acid trapping for triacylglycerol synthesis in FCHL adipose tissue and increased fatty acid flux towards other organs. In muscle and liver fatty acid and their metabolites can accumulate and induce insulin resistance. For instance, there is evidence that glucose uptake in FCHL skeletal muscle responds insufficiently to insulin stimulation in relation to the rate of fatty acid supply. There is no literature data, however, to suggest that insulin resistance in liver contributes to FCHL expression. It is of interest to investigate this potential mechanism in FCHL, because hepatic lipoprotein production is a function of hepatic insulin sensitivity. For example, insulin normally suppresses hepatic production of A po B-100 lipoproteins, especially the production of triglyceride-rich lipoproteins. Another factor that can affect hepatic lipoprotein production can be accumulation of fat in the liver (fatty liver).

The very-low density lipoprotein-triacylglycerol (VLDL-TG) pathway, which reffects the metabolism of Apo B-100 lipoproteins, carries most of plasma triglycerides in the fasting state. In this thesis, we show that alterations in the VLDL-TG pathway contribute to the varied expression of FCHL. 
Very low-density lipoprotein (VLDL) and low-density lipoprotein (LDL) each consist of physicochemically and metabolically distinct subclasses. Our FCHL study sample showed a clear bimodal distribution of LDL size. About half of the subjects presented with large buoyant $\mathrm{LDL}$, designated as "pattern $\mathrm{A}$ ". The other half had small dense $\mathrm{LDL}$, or "pattern $\mathrm{B}$ " (chapter 2). Subsequently, we assessed whether pattern A or B associate with aggregate metabolic phenotypes. Subjects with predominantly large, buoyant LDL showed a hypercholesterolemic phenotype (Fredrickson Ila) and the highest Apo B levels. Subjects with predominantly small dense LDL showed a hypertriglyceridemic (Fredrickson Ib or IV), low HDL cholesterol phenotype, with moderately elevated Apo B, total cholesterol level, and LDL cholesterol level. The presence of increased numbers of VLDL1 particles accounted for the hypertriglyceridemia, and could explain the formation of sd LDL and the low HDL cholesterol in the latter subjects. Noteworthy, the amount of VLDL1-TG correlated positively with plasma insulin in pattern B FCHL subjects, which indicated that in these subjects hepatic (over)production of VLDL1-TG could result from insulin resistance. Therefore, the existence of distinct metabolic phenotypes in FCHL can relate to VLDL particle metabolism.

Factors that can lead to fat accumulation in liver have been reported in FCHL. These include increased fatty acid fluxes, and enhanced synthesis of fat in liver (lipogenesis de novo) due to activation of transcription factors in response to increased substrate availability and hyperinsulinemia. We examined the prevalence of nonalcoholic fatty liver disease in FCHL patients referred to the outpatient clinics of the Maastricht University Hospital, by ultrasonography (chapter 3). We observed a substantial prevalence of nonalcoholic fatry liver disease in FCHL patients, including all of those with elevated plasma triglyceride levels. Fatty liver can contribute to increased production of triglyceride-rich particles through increased avalability of substrate for incorporation in VLDL.

We hypothesize that plasma lipoprotein phenotypes in FCHL can be determined by differences in metabolic profile between individuals, which can relate to insulin resistance and the presence or absence of fat accumulation in liver (chapter $2,3,4$ and 5). These differences can result from environmental influences or genetic variants, or a combination of both. Future studies are needed to confirm this hypothesis.

Hyperlipidemic conditions have been associated with a prothrombotic state, but the nature of this association has not been well studied in FCHL. Therefore, we studied the 
activity profile of the blood coagutation system, and its relationship to the lipid phenotypes in FCHL. All hyperlipidemic FCHL subjects exhibited a prothrombotic phenotype, including elevated levels of several factors of the coagulation system (chapter 4). This observation can be interpreted as an activation of the vascular endothelium in response to plaque burden and can relate to the observed susceptibility of premature acute coronary events in FCHL. Increased plasminogen activator inhibitor 1 (PAI-1) activity was specifically observed in hypertriglyceridemic FCHL subjects with features of the metabolic syndrome. Increase in PAI- 1 activity indicates impaired fibrinolysis and may provide an additional cardiovascular risk factor in these subjects.

FCHL subjects exhibit a complex phenotype with a high prevalence of the metabolic syndrome. FCHL subjects with the full-blown metabolic syndrome typically showed numeric expansion of the plasma VLDL1 lipoproteins. VLDL1-TG was the major determinant of the variability of plasma triglycerides. (chapter 5). One explanation of VLDLI plasma expansion. in FCHL can be overproduction of VLDLI that occurs against a background of insulin resistance. It is of interest that patients with type $2 \mathrm{DM}$, another disorder with high prevalence of the metabolic syndrome, exhibit hepatic production of much larger, lipid enriched VLDLI particles than controls, as reported elsewhere. By contrast, VLDLI particles in FCHL subjects were of normal size. Therefore, it is likely that biochemical and genetic differences in hepatic lipid metabolism exist between FCHL and other forms of the metabolic syndrome or at least subjects with type 2 DM. The molecular mechanisms of VLDL overproduction in FCHL remain to be evaluated.

Treatment with atorvastatin $40 \mathrm{mg}$ per day, at bedtime, for 8 weeks effectively lowered plasma lipids in hypertriglyceridemic $\mathrm{FCHL}$, without concomitant changes in surrogate markers of insulin resistance, stch as plasma insulin, HOMA or plasma FFA concentrations (chapter 6 ). However, plasma triglycerides remained elevated $(>2 \mathrm{mmol} / \mathrm{L}$ ) upon therapy in most subjects, with no change in LDL size. HDL cholesterol levels improved but remained low on therapy. Atorvastatin reduced levels of cardiovascular risk markers, particularly PAI. 1 and C-reactive protein (CRP). Noteworthy, no relationship was observed between changes in PAI-1, or CRP and lipid variables. Because of these pleiotropic effects, statins can be important in treatment of FCHL, especially coronary artery disease prevention. 
Potentially, hypertiglyceridemic FCHL patients can have an additional benefit from therapies that target insulin resistance. 


\section{Samenvatting}

Familiaire Gecombineerde Hyperlipidemie (FGH) is een genetische aandoening die gekenmerkt wordt door een verstoord vetmetabolisme. Aangedane personen afkonstig van FGH families vertonen én van de volgende karakteristieken: een verhoogd cholesterolgehalte in het bloed, bekendstaand als Fredrickson type IIa hyperlipidemie; hypertriglyceridemie, bekend als type IV hyperlipidemie of een combinatie van verhoogd cholesterol-en hypertriglyceridemie, ofwel Fredrickson type IIb. Het frequente optreden van hart- en vaatziekten op jonge leeftijd (vóór 60 jaar) is kenmerkend voor FGH families. Personen met FGH laten dikwijls een verhoging van de insuline plasmaspiegel zien met tevens insulineresistentie, abdominale obesitas en een verhoogde bloeddruk. Een plasma fenotype met verhoogde triglyceriden, laag high-density cholesterol (HDL chollesterol), in combinatie met de aanwezigheid van small dense LDL (sd LDL) komt vaak voor in personen met FGH en wordt "het atherogene lipoprotein fenotype" genoemd. Wij hebben dit fenotype en de metabole determinanten ervan bestudeerd.

Uit onderzoek blijkt dat FGH een gevolg kan zijn van een verstoord vetzuurmetabolisme. Aangedane personen mel FGH nemen vetzuren langzamer in hun vetweefsel op en mede daardoor blijven vetzuren na een maaltijd langer in de bloedbaan. Vetzuren die lang blijwen circuleren kunnen metabole verstoringen in andere organen veroorzaken. Voorbeelden hiervan zijn de stapeling van vetzuren en hun metabolieten, die in skelletspier en lever tot verninderde gevoeligheid voor de hormonale werking van insuline kunnen leiden. Het is angetoond dat de door insuline gestimuleerde glucoseopmame in de skeletspier in FGH verminderd wordt, afhankelijk wan het aanbod wan wetzuren. Het is nog niet bewezen dat insulineresistentie in de lever betrokken is bij FGH. Dat is belangrijk om te weten want de lipoproteinenproductie (Apo B-100) door de lever is insulline gevoelig. Bij gezonde personen onderdrukt insuline de productie van lever lipoproteïnen, wooral de productie van triglyceridenrijke very low-density lipoproteine (VLDL1). Vetstapeling (vetlever, NASHy in de lever kan ook de lipoproteïnenproductie beïnvloeden. Ook dit werd onderzocht in een FGH patièntenpopulatie, afkomstig uit de polikliniek van het Academisch Ziekenhuis Maastricht. 
De very low-density lipoprotein-triacylglycerol (VLDL-TG) pathway weerspiegelt het metabolisme van plasma Apo-100 lipoproteïne en triglyceriden tijdens de gevaste (nuchtere) toestand. In dit proefschrift hebben we aangetoond dat veranderingen in the VLDL-TG pathway de expressie van FGH beïnvloeden.

Very low-density lipoprotein (VLDL) and low-density lipoprotein (LDL) bestaan uit subklassen, die verschillend zijn in metabool en fysisch-chemisch opzicht. Er was een duidelijk patroon wan LDL subklassen te zien in onze steekproef van FGH families: er waren personen met grote LDL deeltjes, ook wel "patroon A" genoemd, en personen met kleine (small dense, sd) LDL deeljes, ofwel "patroon B" (hoofdstuk 2). Wij onderzochten wat de verschillen waren tussen "patroon $A$ " en "patroon B" patiënten. Personen met patroon $A$ waren hypercholesterolemisch (Fredrickson IIa) en hadden een sterk verhoogd plasma Apo B gehalte. Personen met patroon B waren hypertriglyceridemisch (Fredrickson IIb or IV) en hadden laag HDL cholesterol, in combinatie met matig verhoogde Apo B, totaal cholesterol en LDL cholesterol waarden. Hypertriglyceridemie in deze personen werd veroorzaakt door toename in plasma VLDL1 deeltjes. VLDL 1 wisselt triglyceriden wit met LDL en HDL deeltjes, en een overmatige uitwisseling kan tot gevolg hebben dat er een verlaging van plasma HDL cholesterol en sd LDL stapeling optreedt. Tevens correleerde VLDLI-TG met plasma insuline. Dat kan betekenen dat een (overmatige) VLDL1 productie in personen met patroon B een gewolg kan zijn van insulineresistentie. Bowendien lijkt het VLDL metabolisme de aanwezigheid van aparte fenotypen in FGH te kunnen verklaren.

Een groot aanbod van vrije vetzuren kan bijdragen aan de vetstapeling in de lever. Activatie van transcriptie factoren door hyperinsulinemie speelt ook een belangrijke rol bij de vetstapeling. Wij bestudeerden de prevalentie van vetlever bij FGH patiënten die afkomstig waren van het Academisch Ziekenhuis Maastricht (hoofdstuk 3). Het bleek dat de prevalentie van vetlever bij FGH aanzienlijk groter was dan bij de controlepopulatie, eveneens afkomstig van het Academisch Ziekenhuis Mastricht. Bovendien bleken alle FGH patiënten met een vetlever verhoogde plasma triglyceriden te hebben. Dit kan betekenen dat vetstapeling in de lever bijdratagt aan de overmatige productie van grote triglyceridenrijke VLDL1, door de vergrote beschikbaarheid van substraat voor VLDL synthese. Uiteraard is verder onderzoek in een grotere studie populatie nodig on te begrijpen of deze waarneming bevestigd kan worden. 
Wij veronderstellen dat plasma lipoproteine fenotypen in FGH onstastn door verschillen in het metabole profiel tussen individuen. Omgevingseffecten of genetische variatie, alleen of in combinatie, dragen bij aan het ontstaan van de FGH fenotypen door verschillen in insulineresistentie te veroorzaken, en mogelijk mede vetstapeling in de lever te veroorzaken (hoofdstukken 2,3 en 5 ).

Subklinische activatie van de bloedstolling komt vaak voor in hyperlipidemische omstandigheden, maar deze associatie bleek onvoldoende onderzocht te zijn in $\mathrm{FGH}$. We bestudeerden de activiteit van het bloedstollingssysteem in relatie tot de lipoproteine fenotypen in FGH. Alle aangedane FGH personen bleken een verhoogd plasma gehalte van een of meerdere stollingsfactoren te vertonen (hoofdstuk 4). Deze subklinische activatie van de stollingssysteem kan een belangrijk rol spelen bij het ontstaan van de symptomen van harten vaatziekten in FGH. Een verhoogd plasmagehalte van plasminogen activator inhibitor-1 (PAI-1) was karakteristiek voor hypertriglyceridemische FGH personen met metabool syndroom. Stijging van PAI- 1 activiteit duidt op een verstoorde fibrinolyse en dit kan extra risico op hart- en vaatziekten bij deze personen vormen. Op dit moment is er weinig bekend over een specifieke behandeling hiervan.

Aangedane FGH personen tonen een complex fenotype met verhoogde prevalentie van het metabole syndroom. Een verhoogde VLDL1 plasmaspiegel is kenmerkend voor FGH personen met het metabole syndroom en kan leiden tot hypertriglyceridemie (hoofdstuk 5). Insulineresistentie is waarschijnlijk de oorzaak van overmatige VLDLI productie in personen met FGH en het metabole syndroom. Het is interessant te weten dat personen met type 2 DM, die ook een verhoogd risico op het metabole syndroom hebben, een overmatige leverproductie tonen van VLDL1 deeltjes, die veel groter (triglyceriderijker) zijn dan VLDL1 deeltjes bij gezonde personen. Dit in tegenstelling tot FGH, waar het vetgehalte van de VLDLI. deeltjes bij de FGH patiënten vergelijkbaar was met dat van controles. Die observatie geeft aan dat er verschilten in vetmetabolisme in de lever kunnen bestaan tussen FGH en andere vormen van het metabole syndroom, tenminste met type 2 DM. Deze verschillen moeten nog worden bestudeerd.

Plasmalipiden concentraties, vooral cholesterol en LDL cholesterol, in hypertriglyceridemische FGH personen daalden met atorvastine behandeling ( $40 \mathrm{mg} / \mathrm{d}$ ), na 8 weken monotherapie, maar er was geen verandering in surrogat markers voor 
insulineresistentie zoals plasma insuline, vrije vetzuren en HOMA (hoofdstuk 6). Ook plasma triglyceriden bleven verhoogd ( $>2 \mathrm{mmol} / \mathrm{L}$ ) in de meeste patiënten en de LDL deeltjes bleven klein. HDL cholesterol steeg met therapie, maar bleef laag. Een verklaring hiervoor kan zijn dat plasma triglyceriden, de grootte van LDL deeltjes en HDL-cholesterol nauw verband houden met het onderliggende ziekteproces in FGH. Het plasmagehalte van PAI-1 en Creactive protein (CRP) daalde met atorvastatine behandeling. Er was geen verband tussen de veranderingen in lipiden en PAI-1, of CRP. Vanwege gecombineerde effecten blijven statines belangrijk voor de preventie en de behandeling van hart - en vaatziekten bij FGH patiënten. Behandeling van de insulineresistentie kan extra voordeel opleveren bij hypertriglyceridemische FGH patiënten omdat dat het onderliggende ziekteprocess aangrijpt. 
Acknowledgements 
Dear Tjerk, firstly I would like to thank you. It was our first encounter during my internship as an Erasmus student at AZM that stimulated me to go into research. Later, with your help I was able to join the MME lab as a Marie-Curie scholar. Your enthusiasm was catching and I enjoyed very much working with you. We had interesting and fruitful discussions, which I really missed when you left the lab. I appreciate very much that I could talk freely with you about things that troubled me, and above all you always managed to help me find a solution.

Hugo, a question on coagulation that bothered me enough to send you an email turned into a successful collaboration project. You are a very considerate person that I am glad to know. Thank you very much for helping me carry my research project until the end.

Marleen, Carla, Martijn, Steven, Vicky, Petra, Josephine, Margee, Margriet and Mohammed; three years slipped away like a snip of the fingers. It seems a short period of time, but looking back I realize how much I have learned and achieved in this period. Because for me, the "selfinsecure" eastern-European, my PhD research project at Maastricht University was not only the necessary - or well planned - step in the career, but it was an opportunity and a life-long experience. I realize how much all you people mean to me, how much you have helped and encouraged me on the way. I appreciate so much to have been able to work with you all!

Marleen, special thanks for your considerable contribution to this work!

Martijn, thank you for being a great friend; you made a fantastic best man too. We have had a wonderful time together. I enjoyed all our discussions on science, medicine, love and all the rest in which we can get lost for hours. We must have been a funny sight at times; rushing through beautiful Prague, deeply engulfed in a discussion on the unclear border between type 2 DM and FCHL, totally unaware of the surrounding. And all the crazy things that we did together; take for example the TNO project - it was tough, but we managed. You also never questioned my driving skills. You are a precious person, 1 admire your passion for the things that you do. 1 believe that you are going to make a very good researcher and a wonderful doctor. Thank you for all your trust and support and please, forgive me the tears that you had to witness at times. 
Vicky, you are a considerate and trustworthy person. I chose you to be my paranimpf because I. wanted it to be somebody that I can totally rely on and that is definitely YOU!

Dear Steven and Dennis, it was lots of fun doing research side by side with you. I wish both of you a successful finish of your PhD projects.

Jaap, thank you that you helped me go through all the procedures to become a "legal alien" in The Netherlands. Without your help I am not sure where it all would have ended up, because when the police requires a heallth insurance in order to issue a residence permit, and the health insurance company wouldn't give a health insurance without a valid residence permit, you need a friend (if possible someone that works part-time for the police) to break this vicious circle. You organized my Dutch language course and thanks to that I was able to pass the Dutch state exam. I appreciate very much that you agreed to be my paranimf, PAPAnimf as you rather feel, and to help with organizing the reception (very thankful again because I don't like organizing).

II want to express my gratitude to Rene van Oerle for training me to perform ELISA, to Jose for making the measurements on Factor XIla in our study on prothrombotic markers, to Rene and Iris for their help in performing the adipose tissue biopsy, to all the patients that participated in the LIGEAT study, to Simone Timmer and to associate professor Margarita Tzonzoerova.

Lеси, вннаги сьм те смятала за мой най-близык приятел, за много важен човек от моето мрастване. Времето в англйската бене страхотно, но един доста труден период, в който актинн тьрсин н намнраш себе сн. Хората, с конто те свьрзва сьдбата тогана често остават приятели за цял живот. Ти беше нешо повече, имах чувството че ни свързва съкровена тайна. Кой би повярвал, фе пьтмшата ни отново ще се пресекат, и то по такыв невероятен нацин. Без твоя талант моята книжка едва ли иеше да стане толкова хубана. Бтагодаря ти от все сьрце за дългите часове на труд, които ми посвети и за. безкрайното тьрпение. Твойте илострапин са невероятен подарьк и истинско 6oratetBo! 
Клия, благодаря ти, че си такъ всеотдаен приятел, човек винаги може да раззита на те6!

Мило семейство, благодаря ви за обичта, грижите доверието. Благодаря ви от все сьрне мила мамо, татко, Таня, бабо, дядо, вуйчо, вуйне, Момцил н Страхил. Врқзката, която нщ свързва е много сшнна, вашата обқч и доверие са ми давали много сниа в трудни моменти. Често не ми беше лесно. Мъчно ми е, че баба Ани и дядо Гопо не дочакаха защитата, щяха да бъдат много горди. Спомням си колко се радваха за дипломирането ми. Тимо, много се радвам, че си част от нашето семейство и се грижнш за Таня с толкова много обич.

Sander, I appreciate your efforts to get Andreas and me familiar with the Dutch culture and to make our stay in Maastricht cozier. Even at this very moment that I am writing this I am wearing your special Dutch present, "oranje klompen".

Dear Andy, beloved, the last but not the least I would like to thank you for taking up the challenge to come and live with me in the Netherlands. I am not sure I would be able to go through all this alone. Only you know how difficult it was during the last three years. The stress with the residence permit, the work permit, and the prolongations were a real trial for our relationship. Our love grew only stronger. Thank you for your love and care. I know that at times I managed to confuse your German mind with my Bulgarian feeling for fatality. Forgive me this, and thank you for your belief in me, and all the comfort. I really meeded that. Now that all tough issues are settled and it's all done with, I finally believe that we can have a future. LOVE is the key.

Thank you all from all my heart!

Yours, 


\section{List of Publications}

Tull papers.

Georgieva AM. wan Greevenbroek M.M.I., Krauss R.M., Brouwers M.C.G.., Vermeulen V.M.M.-3., Robertus-Teunissen MG, wan der Kallen C.J.H, and de Buin T.W.A.

Subctasses of low-aensity lipoprotein and very low-density lipoprotein in familial combined hypertipidemia: relationship to multiple lipoprotein phenotype.

Arterioscler Thromb Vase Biol. 2004 Apr;24(4):7449.

de Brum T.W.A. Georgieva A.M.,Brouwers M.C.G.J., Heitink M.V., van der Kallen C.J.H., and van Greevenbroek M.M.J.

Radiologicat evidence of non-alcoholic fatty liver disease in familial combined hyperlipidemia.

An IMed. 2004 Jun 15; $116(12): 847-9$.

Georgieva A.M., ten Cate H., Keulen E.T. P, van Oerle R., Govers-Riemsiag J.W.P., Hamulyak K., van der Kallen C.J.H., van Greevenbroek M.M.J.and de Bruin T.W.A. Prothrombotic markers in familial combined hyperlipidemia: Evidence of endothelial cell activation and relation to metabolic syndrome.

Atherosclerasis 2004; $175(2): 345-351$.

Georgieva A.M., van Greevenbroek M.M.J., Brouwers M.C.G.J., wan der Kallen C.J.H., and de Bruin T.W.A.

VLDL subclass distribution in familial combined hyperlipidemia: Relationship to hypertriglyceridemia and metabolic syndrome.

Submitted. 
Browwers M.C.G.J., Georgieva A.M. Bilderbeek-Beckers M.L.A., Robertus-Teunissen M.G., van der Kallen C.J.H., van Greevenbroek M.M.J., and de Bruin T.W.A.

Role of Fatty Liver in Dyslipidemia in Familial Combined Hyperlipidemia: Modulator of Cardiovascular risk?

Submitted.

Brouwers M.C.G.J , Bilderbeek-Beckers M.L.A., Georgieva A.M., wan der Kallen C.J.H., van Greevenbroek M.M.J, and de Bruin T.W.A.

Role of Obesity in Nonalcoholic Fatty Liver Disease in Famillial Combined Hyperlipidemia Submitted.

\section{Abstracts}

Georgieva A.M., van Greevenbroek M.M.J., Brouwers M.C.G.J, de Bruin T.W.A. et al. VLDL1 rather than VLDL2 number determine plasma triglyceride concentrations in Familial Combined Hyperlipidemia.

Dutch Atherosclerosis Society Symposium,2005, Abstract book, page 28.

Georgieva A.M., van Greevenbroek M.M.J., Brouwers M.C.G.J., de Bruin T.W.A. et al. Accumulation of VLDLI particles in plasma is the main determinant of hypertriglyceridemia. $75^{\text {th }}$ Congress of the European Atherosclerosis Society, 2005, Abstract book, page 135.

Georgieva A.M. vd Kallen C.J.H., de Bruin T.W.A. et al.

Lipitor, inflammation and gene expression in adipose tissue in familial combined hyperlipidemia.

$75^{\text {th }}$ Congress of the European Atherosclerosis Society, 2005, Abstract book, page 20. 


\section{CURRICULUM VITAE}

Name

Anna Marinova Georgieva

Date \& place of birth

20.09.1976, Sofia, Bulgaria

Educational Background, Research and Occupation

$1990-1995$

English Language School, Sofia, Bulgaria

$1995-2001$

Medical University of Sofia, Bulgaria

June-August, 2001

Three months of clinical internship at Maastricht Academic Hospital

November, 2001

Medical Degree

$2002-2005$

PhD student

Laboratory for Molecular Metabolism and Endocrinology, Department of Internal Medicine, Masstricht University The Netherlands

Supervisors: Prof. TWA de Bruin and Prof. H. ten Cate

December, 2002

State examen "Dutch as second language" (NT2)

$2004-2005$

Reviewer for Arteriosclerosis, Thambosis and Vascular

Biology

August, 2005

Associate Program Manager

Excerpta Medica, Elsevier, Amsterdam

The Netherlands (temporary)

\section{Grants and research support}

Erasmus scholarship (2001); Marie-Curie Research grant (2002); Job Cohen Fund grant (2002-2003), Pfizer Grant (2003-2004). 